\title{
Rehabilitationsstandards für die Anschlussheilbehandlung und allgemeine Rehabilitation von Patienten mit einem Herzunterstützungssystem (VAD - ventricular assist device)
}

\author{
Detlev Willemsen $^{1} \cdot$ C. Cordes ${ }^{2}$ - B. Bjarnason-Wehrens ${ }^{3} \cdot$ E. Knoglinger ${ }^{4}$ \\ E. Langheim ${ }^{5} \cdot$ R. Marx ${ }^{6,7} \cdot$ N. Reiss ${ }^{1}$ T. Schmidt ${ }^{1}$ A. Workowski ${ }^{1}$ P. Bartsch ${ }^{1}$. \\ C. Baumbach $^{8} \cdot$ C. Bongarth ${ }^{9}$ H. Phillips ${ }^{10} \cdot$ R. Radke ${ }^{11} \cdot$ M. Riedel ${ }^{12} \cdot$ S. Schmidt $^{2}$. \\ E. Skobel ${ }^{13} \cdot$ C. Toussaint ${ }^{14} \cdot$ J. Glatz ${ }^{5}$ \\ Online publiziert: 16. Februar 2016 \\ (C) Die Autor(en) 2016. Dieser Artikel ist auf Springerlink.com mit Open Access verfügbar.
}

\section{Inhalt}

1 Einleitung: Entwicklung der Rehabilitationsstandards für die Rehabilitation von Patienten mit einem VAD (ventricular assist device) . . . . . . . . . . . . . . 3

2 Rehabilitationsstandards ................4

2.1 Rehabilitationsfähigkeit von VAD-Patienten ......4

2.2 Qualitäts- und Personalvoraussetzungen für die

Rehabilitation von VAD-Patienten . . . . . . . . . . . . . .

2.3 Management von VAD-Systemen . . . . . . . . 7

Dr. med. Detlev Willemsen

DWillemsen@Schuechtermann-Klinik.de

Schüchtermann-Klinik, Ulmenalle 5-11, 49214 Bad Rothenfelde, Deutschland

2 Gollwitzer-Meier-Klinik, Bad Oeynhausen, Deutschland

Institut für Kreislaufforschung und Sportmedizin, Deutsche Sporthochschule Köln, Köln, Deutschland

4 Kirchberg-Klinik, Bad Lauterberg, Deutschland

5 Reha-Zentrum Seehof der DRV-Bund, Teltow, Deutschland

6 MediClin Fachklinik Rhein/Ruhr, Essen, Deutschland

7 Universität Witten/Herdecke, Witten, Deutschland

8 Herz- und Gefäßzentrum Bad Bevensen, Bad Bevensen, Deutschland

9 Klinik Höhenried, Bernried am Starnberger See, Deutschland

10 Reha Parcs Steinhof, Erkrath, Deutschland

11 Christiaan-Barnard-Klinik, Dahlen-Schmannewitz, Dahlen, Deutschland

12 Klinik Fallingbostel, Bad Fallingbostel, Deutschland

13 Rehaklinik „An der Rosenquelle“, Aachen, Deutschland

14 m\&i Fachklinik Herzogenaurach, Herzogenaurach, Deutschland
2.4 KardiologischeDiagnostikwährend derRehabilitation von VAD-Patienten . . . . . . . . . . . . 11

2.5 Leistungsdiagnostik nach VAD-Implantation . . . . 13

2.6 Umgang mit Herzrhythmusstörungen sowie mit ICDCRT- und Herzschrittmacheraggregaten während der Rehabilitation von VAD-Patienten . . . . . . . . 14

2.7 Besonderheiten der Medikation während der Rehabilitation von VAD-Patienten . . . . . . . . 16

2.8 Hygiene, Pflege und Monitoring bei VAD-Patienten in der Rehabilitation . . . . . . . . . . . . . . 18

2.9 Anwendung der ICF bei VAD-Patienten . . . . . . . . 20

2.10 Bewegungstherapie im Rahmen der Rehabilitation nach VAD-Implantation . . . . . . . . . . . 22

2.11 Ergotherapie während der Rehabilitation von VAD-Patienten .................26

2.12 Psychologische Probleme und Lebensqualität sowie deren Erfassung durch Fragebögen bei VAD-Patienten ................. 27

2.13 Besonderheiten bei der Ernährung von VADPatienten . . . . . . . . . . . . . . . . . . . . . 29

2.14 Schulung von Patienten: Aufgaben des Rehabilitationsteams ... . . . . . . . . . . 32

2.15 Sozialarbeit und Entlassungsmanagement der Rehabilitationskliniken sowie Beratungsgrundlagen für Arbeiten, Fahren und Reisen mit einem VAD . 34

2.16 Standards für den Umgang mit Notfallsituationen in der Rehabilitation von VAD-Patienten . . . . . . . 39

2.17 Ethische und palliativmedizinische Aspekte bei der Betreuung von VAD-Patienten unter Beachtung juristischer Vorgaben . . . . . . . . . . . . 41

3 Appendix . . . . . . . . . . . . . . . . . 42

3.1 Autorenerklärung . . . . . . . . . . . . . . 42

3.2 Weitere Mitwirkende . . . . . . . . . . . . . . . . 42

3.3 Abkürzungsverzeichnis . . . . . . . . . . . . . . 42 Literatur 


\section{Rehabilitation standards for follow-up treatment and rehabilitation of patients with ventricular assist device (VAD)}

Abstract The increasing use of ventricular assist devices (VADs) in terminal heart failure patients provides new challenges to cardiac rehabilitation physicians. Structured cardiac rehabilitation strategies are still poorly implemented for this special patient group. Clear guidance and more evidence for optimal modalities are needed. Thereby, attention has to be paid to specific aspects, such as psychological and social support and education (e.g., device management, INR self-management, drive-line care, and medication).

In Germany, the post-implant treatment and rehabilitation of VAD Patients working group was founded in 2012. This working group has developed clear recommendations for the rehabilitation of VAD patients according to the available literature. All facets of VAD patients' rehabilitation are covered. The present paper is unique in Europe and represents a milestone to overcome the heterogeneity of VAD patient rehabilitation.

Keywords Cardiac rehabilitation - Heart assist devices · Heart failure $\cdot$ Patient education $\cdot$ Ventricular dysfunction

Geschlechterneutrale Formulierung: Aus Gründen der besseren Lesbarkeit wird auf die gleichzeitige Verwendung männlicher und weiblicher Sprachformen verzichtet. Sämtliche Personenbezeichnungen gelten gleichermaßen für beiderlei Geschlecht.

\section{Einleitung: Entwicklung der Rehabilitationsstandards für die Rehabilitation von Patienten mit einem VAD (ventricular assist device)}

Herzinsuffizienz bildet nach der chronischen ischämischen Herzkrankheit und dem akuten Myokardinfarkt die dritthäufigste Todesursache in Deutschland [1]. Insgesamt starben im Jahr 2013 in der Bundesrepublik Deutschland 45.815 Menschen an den Folgen dieser Krankheit. Ebenso bildete Herzinsuffizienz im selben Jahr mit 396.380 Krankheitsfällen - abgesehen von den Kindsgeburten - die häufigste Ursache für einen stationären Krankenhausaufenthalt [2].

Herzinsuffizienz kann durch eine Vielzahl von Grunderkrankungen verursacht werden. Den wichtigsten und kausalen Ansatz bei der Behandlung stellt die Therapie der jeweiligen Grunderkrankung dar. Ist das Voranschreiten der Herzinsuffizienz trotz optimaler Pharmakotherapie unvermeidbar, wird bei fortgeschrittenen Krankheitsstadien in
Tab. 1 Entwicklung der VAD-Implantationsstrategie in den Vereinigten Staaten zwischen Januar 2008 und Dezember 2013, modifiziert nach Kirklin et al. [7]

\begin{tabular}{llll}
\hline & & \multicolumn{2}{l}{ Implantationszeitraum } \\
$\begin{array}{l}\text { Device-Strategie im } \\
\text { Implantationszeitraum }\end{array}$ & & $2008-2010(\%)$ & $2011-2013(\%)$ \\
Brigde to transplant & (BTT) & 78,2 & 53,8 \\
Destination therapy & (DT) & 19,6 & 41,6 \\
Bridge to recovery & (BTR) & 1,1 & 1,0 \\
Andere & & 1,4 & 0,8 \\
\hline
\end{tabular}

Spezialambulanzen letztendlich die Indikation zur Herztransplantation (HTX) geprüft. Zum Jahresende 2014 waren in Deutschland laut Eurotransplant [3] insgesamt 858 Patienten für eine HTX gelistet - zur Verfügung standen im selben Jahr jedoch nur 409 Spenderherzen [4]. Diese Bedarfsproblematik soll durch Implantation von Herzunterstützungssystemen (,ventricular assist devices“ - VAD) entschärft werden [5].

In Abhängigkeit von der Krankheitssituation stehen unterschiedliche Systeme zur Verfügung. Mit pulsatilem oder kontinuierlichem Fluss unterstützen elektromechanisch arbeitende Blutpumpen aus Titan die erkrankten Herzkammern und normalisieren damit weitgehend den inadäquaten Kreislauf, indem sie das Blut über einen Stutzen aus den Herzkammern ansaugen und es über eine Kunststoffprothese in die Aorta und/oder die Pulmonalarterie befördern. Informationsaustausch und Energieübertragung erfolgen dabei über ein Verbindungskabel vom intrakorporal gelegenen Aggregat zu extrakorporalen tragbaren Steuerelementen. Deutschlandweit wurden im Jahr 2014 insgesamt 957 derartige Pumpen eingebaut [6].

Anfangs wurden „Kunstherzen“ nur als Überbrückungstherapie („,bridge to transplant") in der Wartezeit für eine HTX implantiert. Nach stetiger Weiterentwicklung der Geräte ist der Einsatz von VAD inzwischen auch als dauerhafte Alternative (,destination therapy“) zur HTX möglich, wie aktuelle Zahlen aus den Vereinigten Staaten belegen (Tab. 1; [7]). Zwischen 2011 und 2013 wurden demnach (in den USA) über $40 \%$ der implantierten Pumpen unter der Strategie einer Langzeit- und Dauerlösung verwendet mit steigender Tendenz. In einem weiteren Einsatzgebiet können VAD auch als nur zeitlich befristete Kompensation bei akuten Herzmuskelkrankheiten mit guter Erholungsaussicht zum Einsatz kommen (,bridge to recovery“).

Bezogen auf die Haltbarkeit der mechanischen Pumpsysteme werden maximale Einsatzzeiten von annähernd 8 Jahren erreicht. Nach einem dann möglicherweise notwendig gewordenen Pumpenwechsel können inzwischen Überlebensspannen der VAD- Patienten von bis zu 14 Jahren beobachtet werden [8].

Aufgrund der verlängerten Haltbarkeit der VADSysteme und der erreichten Fortschritte der letzten Jahre (z. B. kleinere Devices, verbesserte Lebensqualität), 


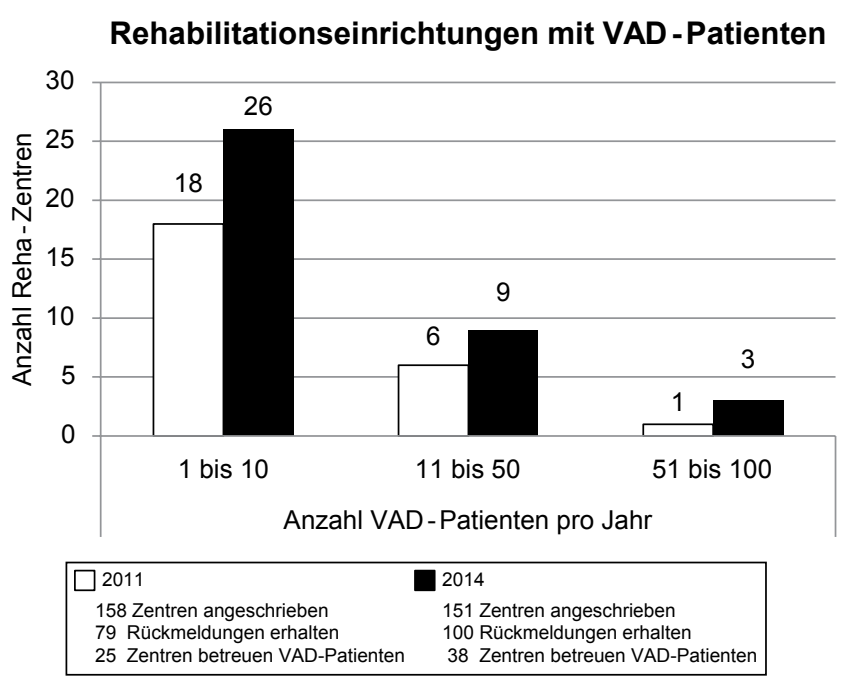

Abb. 1 Anzahl der Rehabilitationseinrichtungen in Deutschland, die VAD-Patienten betreuen, unterteilt nach jährlichem Patientenaufkommen [9]

wird die Akzeptanz von VAD-Systemen zukünftig weiter steigen. Ein nachfolgend erhöhtes Patientenaufkommen von rehabilitationsfähigen und -bedürftigen VAD-Patienten ist bereits jetzt in den deutschen Rehabilitationskliniken zu beobachten [9]. Im Jahr 2014 erhielten in Deutschland insgesamt 517 VAD-Patienten eine Rehabilitationsmaßnahme in einer kardiologischen Rehaklinik. Dies entspricht einer Patientenzunahme von $58 \%$, verglichen mit Zahlen aus 2011. Die Anzahl der aufnehmenden Kliniken in Deutschland erhöhte sich um $52 \%$ auf nun 38 Zentren. Das bedeutet, dass heutzutage bereits jede 4. kardiologische Rehaklinik VAD-Patienten aktiv rehabilitiert. Die absolute Zahl der betreuten Patienten variiert unter den Kliniken allerdings erheblich (Abb. 1; [9]).

Für die Entwicklung einer angepassten Versorgungssituation dieser Patienten ist ein einheitlicher Behandlungspfad wünschenswert. Die „European Society of Cardiology“ (ESC) und die „International Society for Heart \& Lung Transplantation" (ISHLT) haben bereits Empfehlungen [10-12] bzw. Leitlinien [13] zur Behandlung von Patienten mit einem Herzunterstützungssystem erarbeitet; diese sind jedoch noch überwiegend vage formuliert und nur bedingt auf den nationalen Kontext übertragbar. Auch gilt es, Vorgaben eines erstmalig neu im Jahr 2015 eingeführten Qualitätssicherungsprogramms zu erfüllen, wonach Überlebensraten für die ersten 2 Jahre bei dieser Patientengruppe definiert sind (70 bzw. 60\%) und Fehlfunktionen der eingesetzten Systeme nur in weniger als 5\% der Fälle auftreten dürfen [14].

Im Frühjahr 2012 trafen sich erstmals Ärztliche Leiter kardiologischer Rehabilitationskliniken mit Behandlungserfahrung bei der Betreuung von Trägern mechanischer Kreislaufsysteme, um auf Expertenebene Erfahrungen aus- zutauschen. Zur Teilnahme eingeladen waren alle deutschen Fachkliniken mit ihren interdisziplinären Teams. Aus dieser Gruppe heraus gründete sich ein an die DGPR (Deutsche Gesellschaft für Prävention und Rehabilitation von HerzKreislauferkrankungen e. V.) assoziierter Arbeitskreis (AKVAD). Ziel dieses AK-VAD war u. a. die Erarbeitung eines nationalen Behandlungsstandards für die kardiologische Rehabilitation von VAD-Trägern mit Publikation der Ergebnisse in einem Positionspapier (Evidenzgrad: Level C).

Für die praktische Umsetzung der Behandlungsstandards im Rehabilitationsalltag bietet das Priorisierungsschema MoSCoW [15] eine Orientierungshilfe. MoSCoW steht für: M - MUST (unbedingt erforderlich), S - SHOULD (sollte umgesetzt werden, wenn alle MUST-Anforderungen trotzdem erfüllt werden können), C - COULD (kann umgesetzt werden, wenn die Erfüllung von höherwertigen Anforderungen nicht beeinträchtigt wird), W - WON'T (wird diesmal nicht umgesetzt, aber für die Zukunft vorgemerkt) [15].

Das Positionspapier liegt nun in einer ersten Version vor. Die zu entwickelnden Behandlungsstandards sind eingeteilt in 17 gesonderte Themenblöcke und wurden gemeinsam nach Expertenkonsens auf der Basis praktischer Erfahrungen und wissenschaftlicher Literaturrecherchen in Zusammenarbeit mit zuweisenden Implantationszentren der Herzchirurgie erarbeitet.

Mittelfristig können die jetzt formulierten Behandlungsstandards eine Grundlage zur Formulierung eines Kenndatensatzes für ein Rehabilitationsregister dieser Patientengruppe bieten.

\section{Rehabilitationsstandards}

\subsection{Rehabilitationsfähigkeit von VAD-Patienten}

Nach Implantation eines VAD besteht in aller Regel ein Bedarf für eine kardiologische Anschlussheilbehandlung (AHB) in einer spezialisierten Einrichtung, die eine entsprechende fachliche Qualifikation sowie angemessene personelle und apparative Ausstattungen aufweist.

Die Rehabilitation umfasst neben einem intensiven Trainingsprogramm wegen der meist ausgeprägten körperlichen Beeinträchtigungen eine sorgfältige Schulung im Umgang mit dem VAD, dem Verbandswechsel der Driveline und der Steuerung der Antikoagulation sowie eine psychologische und soziale Beratung und Betreuung. Wenn möglich, sollten dabei auch die Angehörigen in den Rehabilitationsprozess eingebunden und vor allem an den Schulungen beteiligt werden. Wichtigstes Ziel im Rahmen der Anschlussrehabilitation ist das Erreichen einer möglichst eigenständigen häuslichen Versorgung. 
Im Langzeitverlauf kann in Einzelfällen eine erneute Rehabilitation zur Wiederherstellung der Teilhabe mit Verbesserung von Lebensqualität und Lebenserwartung erforderlich sein, vor allem nach Komplikationen wie Thrombosen, Schlaganfällen oder Infektionen. Dabei ist bei besonderen beruflichen Problemlagen eine medizinischberufsorientierte Rehabilitation (MBOR) sinnvoll [16]. Diese ist jedoch im Rahmen einer Anschlussrehabilitation im Normalfall noch nicht möglich.

Die Rehabilitationsfähigkeit [17] nach VAD-Implantation hängt im Wesentlichen vom klinischen Zustand des Rehabilitanden (somatisch, psychisch und kognitiv) und den medizinischen Begleitumständen (z. B. bei weiteren Einschränkungen durch Begleiterkrankungen) ab.

Kardiologische Rehabilitationskliniken mit zusätzlichen Fachbereichen (z. B. für Orthopädie oder Neurologie) sind besonders geeignet zur Aufnahme von VAD-Patienten mit entsprechenden Komorbiditäten.

Insbesondere bietet eine zusätzliche neurologische Rehabilitationsabteilung weitere umfangreiche Möglichkeiten zur Behandlung von VAD-Patienten mit vorbestehenden bzw. auftretenden schwerwiegenden neurologischen Komplikationen.

Eine uneingeschränkte Rehabilitationsfähigkeit besteht dann, wenn die Rehabilitanden dem komplexen RehaProgramm körperlich und kognitiv gut folgen können und eine weitgehende Selbstversorgung in der Rehabilitationseinrichtung vor allem im Bereich der ATL (Aktivitäten des täglichen Lebens) gegeben ist. Hier sind die Rehabilitationsziele einer komplett eigenständigen Versorgung und des zuverlässigen Umgangs mit dem Assist-Device und der oralen Antikoagulation gut zu erreichen.

Eine eingeschränkte Rehabilitationsfähigkeit liegt vor, wenn die Erkrankung bzw. Begleiterkrankungen nur zu einer bedingt selbstständigen Versorgung befähigen und im Rehabilitationsverlauf dadurch ein zusätzlicher Pflegebedarf bei dem ohnehin schon erhöhten Versorgungs- und Betreuungsaufwand der VAD-Patienten besteht.

Dieses kann insbesondere bei psychisch-kognitiven Problemen der Fall sein. Im somatischen Bereich stehen vor allem die körperlichen Einschränkungen der Aktivitäten des täglichen Lebens (ATL) im Vordergrund. Diese können aus vielfältigen Gründen beeinträchtigt sein (Paresen, Critical Illness Neuropathie, Infektionen, Sturzgefahr, allgemeine Schwäche, Amputationen, Schmerzzustände etc.).

Eine Besiedlung mit multiresistenten Keimen (z. B. VRE, MRSA, MRGN) muss bei einem entsprechenden Hygienekonzept der Rehabilitationsklinik nicht zu einer Aufhebung der Rehabilitationsfähigkeit führen.

Ebenso kann eine Dialysepflicht bei terminaler Niereninsuffizienz gut in einer Rehabilitationsklinik bewältigt werden, wenn entsprechende Kooperationen mit einem Dialysezentrum bestehen.
Eine aufgehobene Rehabilitationsfähigkeit liegt in der Regel vor, wenn der Patient kognitiv, psychisch und/ oder somatisch den Rehabilitationsmaßnahmen nicht folgen kann. Dies könnte bei permanenter Bettlägerigkeit, ständiger, insbesondere nächtlicher Pflegebedürftigkeit, bei floriden Psychosen, deliranten oder komatösen Zuständen, häufig rezidivierenden Synkopen oder bedrohlichen Rhythmusstörungen, wiederholten schweren kardialen Dekompensationen oder auch akuter Suizidalität der Fall sein. Auch eine weiterhin notwendige akut- oder intensivmedizinische Therapie oder das Vorliegen schwer beherrschbarer Infektionen oder anderer schwerer Begleiterkrankungen kann die Möglichkeiten einer Rehabilitationsklinik übersteigen.

Zur Abschätzung der Rehabilitationsfähigkeit ist eine ausführliche Beschreibung des klinischen Zustands im Reha-Antrag erforderlich. Ein hilfreiches Assessment vor allem zur Einschätzung des individuellen Pflegebedarfs ist daneben die Bestimmung des Barthel-Index [18]. Bei einem Barthel-Index unter 60 ist in der Regel keine Rehabilitationsfähigkeit gegeben. In diesen Fällen sollte eine Entscheidung im Einzelfall und nach Rücksprache mit dem implantierenden Zentrum (ggf. auch mit dem Kostenträger) erfolgen.

Hinsichtlich der Rehabilitationsfähigkeit von VADPatienten ergibt sich somit eine besondere Situation. Um die Versorgungsmöglichkeiten und damit auch den Ressourcenverbrauch für dieses spezifische Patientenkollektiv transparent zu machen, wäre ein Phasenmodell sinnvoll, analog zu den in Deutschland langjährig etablierten Phasen der neurologischen Rehabilitation.

Bei einer Anschlussrehabilitation nach Implantation eines Unterstützungssystems sind viele Rehabilitanden körperlich noch stark geschwächt und nur teilweise mobilisiert. An die medizinische Versorgung und Pflege in der Rehabilitationseinrichtung sind daher besondere und spezifische Anforderungen zu stellen, die mit einem höheren personellen und apparativen Aufwand einhergehen.

Ein Abbruch der Rehabilitation wegen fehlender Rehabilitationsfähigkeit bedeutet für den Rehabilitanden immer eine schwere psychische Belastung. Daher ist im Vorfeld einer Aufnahme eine sorgfältige Evaluation der Rehabilitationsfähigkeit, insbesondere in Bezug auf Begleiterkrankungen und in Bezug auf entsprechende Schwerpunkte der Rehabilitationseinrichtung, von entscheidender Bedeutung.

\subsection{Qualitäts- und Personalvoraussetzungen für die Rehabilitation von VAD-Patienten}

Die Rehabilitation, insbesondere die Anschlussheilbehandlung von Patienten nach einer VAD-Implantation, wurde bisher nur in wenigen darauf spezialisierten 
Rehabilitationskliniken durchgeführt. Die Komplexität der Behandlung von Patienten mit einem VAD erfordert ein hochqualifiziertes multidisziplinäres Rehabilitationsteam. Bei Zunahme der Implantationszahlen sind Voraussetzungen für die Qualifikation des behandelnden Personals sowie für die apparative Ausstattung der Kliniken festzulegen.

Die Klinikausstattung, die Zusammensetzung und Qualifikation des Rehabilitations-Teams entsprechen in der Basis den Strukturanforderungen der allgemeinen kardiologischen Rehabilitation [19-22]. Dieses schließt die räumliche, apparative und notfallmedizinische Ausstattung der Kliniken sowie die Notwendigkeit einer 24-stündigen kardiologischen Hintergrundbereitschaft mit ein.

Für die Rehabilitation von Patienten mit einem VAD gibt es aufgrund der noch kleinen Patientenzahlen bisher keine festen Vorgaben für einen Personalschlüssel. Die Intensität der Betreuung von VAD-Patienten führt jedoch zwangsläufig zu einem erhöhten Personalbedarf aller an der Rehabilitation beteiligten Berufsgruppen. Weitere Voraussetzungen sind ein sehr enger Kontakt sowie möglichst räumliche Nähe zum implantierenden Zentrum, um im Notfall rasche Absprachen und - wenn notwendig Verlegungen zu ermöglichen. Enge Kooperation ist auch bezogen auf weitere, nicht in Rehabilitationskliniken verfügbare diagnostische und therapeutische Leistungen anderer Fachdisziplinen erforderlich. Dies betrifft insbesondere neurologische Konsile, spezielle Laborleistungen etc.

\section{Besonderheiten in der Ausbildung des \\ Rehabilitationspersonals für die Behandlung von VAD- Patienten}

Die allgemeingültigen Ausbildungsvoraussetzungen sowie die Zusammensetzung eines kardiologischen Rehabilitationsteams können den entsprechenden Empfehlungen [19-22] entnommen werden.

Über diese üblichen Voraussetzungen hinaus gibt es jedoch VAD-spezifische Ausbildungs- und Einarbeitungsabläufe, die Berücksichtigung finden müssen.

1. Das den Patienten betreuende Personal (insbesondere im ärztlichen und pflegerischen Bereich) muss mindestens $1 \times$, besser jedoch $2 \times$ jährlich in die Funktion und Handhabung der VAD-Systeme eingewiesen werden. Die Einweisungen sind schriftlich zu dokumentieren. Dies gilt auch für Ärzte im Bereitschaftsdienst aus fachfremden Abteilungen (MUST).

2. Eine Klinik, die VAD-Patienten betreut, muss Personal zur zertifizierten Schulung im Gerinnungsselbstmanagement vorhalten (MUST).

3. Medizinisches und nicht medizinisches Personal muss mindestens $1 \times$, besser jedoch $2 \times$ jährlich eine Reanimationsschulung - orientiert an die Leitlinie der
American Heart Association [23] - erhalten. Hier muss auf VAD-spezifische Probleme bei der Notfallversorgung eingegangen werden (MUST).

4. Die Durchführung insb. der echokardiographischen Diagnostik erfordert spezifische Erfahrungen und Kenntnisse. Diese sollten ggf. durch Hospitation im implantierenden Zentrum abgestimmt werden (SHOULD).

5. Eine Qualifikation zur Kontrolle und ggf. Programmierung von zusätzlich zum VAD implantierten Devices (Fachkunden Herzschrittmacher, ICD) sollte vorhanden sein (SHOULD, siehe Abschn. 2.6. Rhythmus).

6. Vor der Aufnahme eines Patienten mit einem VAD, für das in der Klinik noch keine Einweisung erfolgt ist, muss diese durch die Herstellerfirma bzw. einen Kardiotechniker/VAD-Koordinator vorgenommen und dokumentiert werden (MUST).

Wenn auch als Grundvoraussetzung für die Übernahme in die Rehaklinik die umfassende Einweisung in das VAD und in das Gerinnungsselbstmanagement im implantierenden Zentrum zwingend erforderlich ist, bleibt es Aufgabe der Rehabilitationsklinik, diese Kenntnisse und Fähigkeiten zu dokumentieren, zu überprüfen und zu vertiefen. Dies erfolgt regelhaft im Einzelkontakt und erklärt somit ebenfalls den erhöhten Personalbedarf.

\section{Besonderheiten in der apparativen Ausstattung der Rehabilitationsklinik für die Behandlung von VAD- Patienten}

Die apparative Ausstattung sollte die im Abschn. 2.4 erwähnten diagnostischen Maßnahmen ermöglichen. Aufgrund der oft schwierigen Bedingungen bei der Echokardiographie sollte ein hochwertiges Echokardiographiegerät zur Verfügung stehen. Wegen der Besonderheit beim Messen des Blutdrucks muss die Gefäßdopplertechnik (MUST) vorhanden sein. Röntgenuntersuchungen des Thorax müssen möglich sein (MUST). Die Auswahl von Kontrollgeräten für Herzschrittmacher und ICD orientiert sich an den Implantaten der VAD-Zuweiser (SHOULD). Ein gerätespezifischer VAD-Monitor zum Ablesen und ggf. Programmieren der VAD-Aggregate könnte eine sinnvolle Ergänzung sein (COULD). Eine eventuelle notwendige Umprogrammierung des VAD sollte in enger Absprache mit dem implantierenden Zentrum erfolgen.

Alle Gebrauchsanweisungen der VAD sowie ein Telefonverzeichnis über wichtige Notruf- und Hotline-Nummern der zuweisenden Kliniken und Hersteller müssen an definierten und allgemein bekannten Stellen aufzufinden sein (MUST). Zusätzlich ist es sinnvoll, die Gebrauchsunterlagen und Telefonlisten im Intranet der betreuenden Klinik zu hinterlegen. 
Jede Klinik, die VAD-Patienten betreut, muss grundsätzlich über eine Notstromversorgung verfügen (MUST).

Eine Möglichkeit zur Monitorüberwachung $\left(\mathrm{EKG}, \mathrm{O}_{2}-\right.$ Sättigung) und Notfallbehandlung muss vorhanden sein (MUST).

Eine telemetrische Überwachung der Patienten sollte möglich sein. Ihr Einsatz während der Rehabilitation wird kontrovers diskutiert. Zu unterscheiden sind eine kontinuierliche, eine befristete nächtliche und eine bedarfsabhängige Telemetrieüberwachung (z. B. nach stattgehabten Herzrhythmusstörungen, ICD-Schocks oder hämodynamischer Instabilität).

VAD-Patienten werden in der Rehabilitation mit den Zielen der verbesserten Teilhabe und der Integration in das Alltagsleben behandelt. Eine lückenlose telemetrische Überwachung während der Rehabilitation würde jedoch zu einer starken Verunsicherung und evtl. zu einem Rückschritt im Erreichen dieser Ziele führen. Demgegenüber steht, dass evtl. Notfälle oder auch Dekonnektionen der VAD-Stromzufuhr z. B. in suizidaler Absicht erst zu spät erkannt werden. Diesem Risiko wäre der Patient im alltäglichen Leben in seiner häuslichen Umgebung allerdings in gleicher Form ausgesetzt. Eine mobile, zeitlich begrenzte Telemetrieüberwachung klinisch problematischer Patienten während der Rehabilitation kann jedoch durchaus sinnvoll sein. Bei anhaltend überwachungspflichtigen VAD-Patienten muss die Rehabilitationsfähigkeit kritisch hinterfragt werden.

\subsection{Management von VAD-Systemen}

Das Device-Management von mechanischen Kreislaufunterstützungssystemen ist komplex und stellt sowohl an die behandelnden Ärzte als auch im weiteren Verlauf an den Patienten und sein Umfeld spezielle Anforderungen.

Von den behandelnden Ärzten werden neben Erfahrungen mit der mechanischen Kreislaufunterstützung gute Kenntnisse in den Bereichen Pathophysiologie und Hämodynamik der terminalen Herzinsuffizienz, der Pharmakotherapie, Echokardiographie und Gerinnungssteuerung gefordert, um eine gute Einstellung der Pumpenparameter und eine suffiziente Kreislaufunterstützung vor allem initial, aber auch im Langzeitverlauf zu gewährleisten.

Auf Patientenseite müssen die kognitiven und manuellen Fähigkeiten, das soziale Umfeld und die Wohnsituation ermittelt und ggf. die Bedingungen optimiert werden, damit nach der Operation sowohl dem Patienten selbst als auch seinen Angehörigen und dem Hausarzt die Funktionsweise und der richtige Umgang mit der Technologie (Umgang/ Wechsel der Stromversorgung/Akkumulatoren, Lesen und Verstehen der Anzeigen am Controller) problemlos vermittelt werden können.
Aktuelle Kunstherzsysteme Die heutzutage verfügbaren mechanischen Kreislaufunterstützungssysteme können sowohl zur Unterstützung des linken (,left ventricular assist device“, LVAD) als auch des rechten (,right ventricular assist device“, RVAD) Ventrikels oder auch zur Unterstützung beider Ventrikel (,biventricular assist device“, BVAD) eingesetzt werden. In den meisten Fällen ist es ausreichend, die Pumpfunktion des linken Ventrikels zu unterstützen. Das entsprechende Unterstützungssystem (LVAD) pumpt das Blut aus dem linken Ventrikel in die Aorta [24]. Bei den elektrisch betriebenen Pumpen wird unterschieden zwischen Axial- und Zentrifugalpumpen. Axialpumpen treiben das Blut mit einer rotierenden Schraube voran, Zentrifugalpumpen tun dies mit Hilfe der Zentrifugalbewegung eines Kegels. Bei beiden Geräten fließt das Blut kontinuierlich, das heißt, von diesen Systemen wird kein Puls erzeugt.

Derzeit werden in Deutschland hauptsächlich zwei Linksherzunterstützungssysteme implantiert, das HeartWare ${ }^{\circledR}$ System und das HeartMate II $^{\circledR}$-System [25-30].

Das HeartWare ${ }^{\circledR}$ Ventricular Assist Device ${ }^{\circledR}$ (HVAD $^{\circledR}$; Fa. HeartWare ${ }^{\circledR}$ International, Farmingham, MA, USA) (Abb. 2) ist eine Zentrifugalpumpe mit kontinuierlichem Blutfluss. Die maximale Pumpleistung der $160 \mathrm{~g}$ schweren Pumpe beträgt bis zu $10 \mathrm{l} / \mathrm{min}$. Das hydrodynamische Lager arbeitet mit einer Umdrehungszahl zwischen 1800 und $4000 \mathrm{U} / \mathrm{min}$.

Das HeartMate II ${ }^{\circledR}$ (Fa. Thoratec ${ }^{\circledR}$ Corp, Pleasanton, CA, USA) (Abb. 3) ist das bislang am häufigsten implantierte LVAD mit über 17.000 implantierten Systemen weltweit. Die Axialpumpe wird über die Herzspitze in den linken Ventrikel eingebracht und fördert bis zu $10 \mathrm{l} / \mathrm{min}$. Der Outflow-Graft wird mit der Aorta ascendens anastomosiert. Die $281 \mathrm{~g}$ schwere Pumpe wird in einer präperitonealen Tasche untergebracht. Die längste Laufzeit dieses Systems beträgt über 8 Jahre.

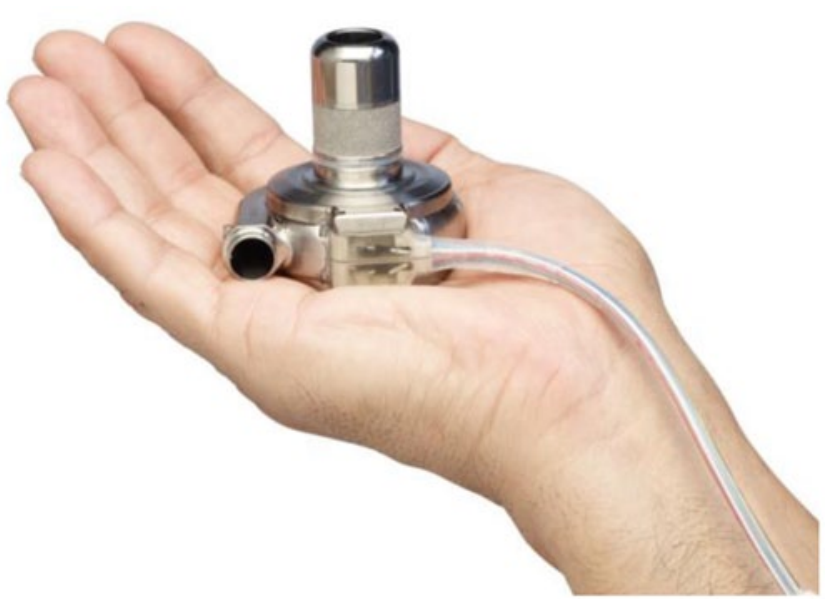

Abb. 2 HeartWare ${ }^{\circledR}$-System [31] 


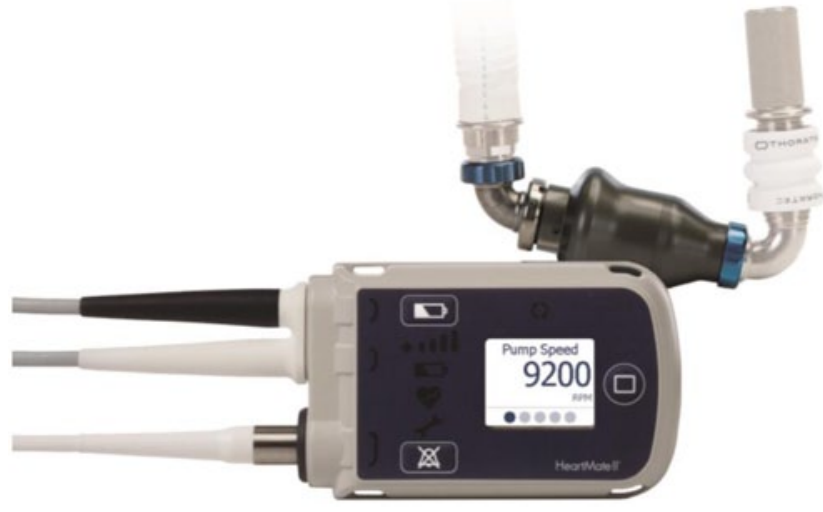

Abb. 3 HeartMate II $^{\circledR}$-System und externer Controller [32]

In Tab. 2 sind die Charakteristika der derzeit in Deutschland am häufigsten implantierten VAD-Systeme dargestellt.

\section{Device-Komponenten}

Die derzeit verfügbaren Linksherzunterstützungssysteme haben neben der implantierten Pumpe extrakorporale Komponenten (Controller mit Anzeige der Pumpenparameter und -alarme und portable Akkus). Für den Betrieb des Systems bei vorhandener Netzstromversorgung, wie etwa zu Hause oder im Auto, sind externe Stromversorgungen (Netzteile, bzw. Spannungswandler) Teil des Systems. Sie werden anstelle eines der beiden Akkus am Steuergerät angeschlossen, sodass weiterhin zwei Stromquellen am Steuergerät vorhanden sind, nämlich ein Akku und die externe Stromversorgung. Die portablen Akkus werden über entsprechende Ladestationen aufgeladen (Abb. 4).

Die Steuereinheit der Blutpumpe ist der sog. Controller, der einen Monitor für die Pumpenparameter, den Fluss (1/ Min.), die Leistung (Watt) und die Umdrehungen (U/Min.) beinhaltet (Abb. 5).

Der Monitor ist in der Regel ein Tablet-PC mit Touchscreen-Bildschirm und firmeneigener Software, der Informationen zum Systembetrieb anzeigt und die Einstellung bestimmter Parameter der Steuereinheit ermöglicht. Bei Anschluss an eine Steuereinheit empfängt der Monitor kontinuierlich Informationen über die Blutpumpe von der Steuereinheit und zeigt sowohl Echtzeit- als auch Verlaufsdaten für die Pumpe an.

Der Controller ist zudem in der Lage, bei einem Systemfehler verschiedene Alarme visuell und auditiv wiederzugeben, um sowohl den Patienten als auch das behandelnde Personal aufmerksam zu machen. Die Alarme besitzen unterschiedliche Dringlichkeitsstufen, welche sich durch unterschiedliche Leuchtsignale und Töne äußern. Durch eine „Driveline“, die von intraperikardial durch die Bauchdecke nach außen zieht, wird die eigentliche Pumpe mit den extrakorporalen Systemkomponenten verbunden.

VADs mit kontinuierlichem Fluss stellen eine besondere Herausforderung beim Monitoring verglichen mit den pulsatilen Unterstützungssystemen dar, da jedes Device spezifische Systemcharakteristika hat.

\section{Festlegung der optimalen Pumpengeschwindigkeit}

Ein elementarer Vorgang beim Device Management von VADs ist die Einstellung der optimalen Flussgeschwindigkeit, da hierdurch eine adäquate Kreislaufunterstützung bei störungsfreiem Pumpenbetrieb erreicht wird.

Es wird eine optimale Umdrehungszahl angestrebt unter Berücksichtigung der Klinik, der Ventrikelgröße, des erreichbaren Cardiac Index unter Vermeidung von Ansaugphänomenen. Die Aortenklappe sollte sich intermittierend öffnen, das interventrikuläre Septum mittig stehen, und eine Minimierung der Mitralklappeninsuffizienz sollte gegeben sein [35]. Am besten erfolgt die Festlegung der idealen Umdrehungszahl durch stufenweises Verändern der Geschwindigkeit unter echokardiographischer und hämodynamischer Kontrolle.

Die Drehzahl wird von dem rechtsventrikulären Volumenangebot begrenzt. Wenn sie zu hoch eingestellt ist, versucht die Pumpe, eine höhere Blutmenge als verfügbar zu fördern. Unter diesen Umständen kann ein ventrikulärer Unterdruck auftreten, der Arrhythmien verursachen oder das Myokard und/oder das Septum beschädigen kann. Zusätzlich kann sich die Hämolyse bei einem schweren Ventrikelkollaps oder bei Verlegung des Einflussweges auf inakzeptable Werte erhöhen.

Tab. 2 Charakteristika der derzeit in Deutschland am häufigsten implantierten VAD-Systeme: HeartMate II ${ }^{\circledR}$ und HeartWare ${ }^{\circledR}[26,30]$

\begin{tabular}{|c|c|c|c|c|c|}
\hline $\begin{array}{l}\text { VAD-Modell } \\
\text { (Firma) }\end{array}$ & Pumpe (Antrieb) & Dauer (Art) & $\begin{array}{l}\text { Lokalität } \\
\text { (Gewicht) }\end{array}$ & $\begin{array}{l}\text { Flussform } \\
\text { (Flussvolumen) }\end{array}$ & Umdrehungsbereich \\
\hline $\begin{array}{l}\text { VAD HeartMate } \\
\text { II }^{\circledR}\left(\text { Thoratec }^{\circledR}\right)\end{array}$ & $\begin{array}{l}\text { Axialrotations- } \\
\text { pumpe } \\
\text { (elektrisch) }\end{array}$ & $\begin{array}{l}\text { Mittel- bis lang- } \\
\text { fristig (komplett) }\end{array}$ & $\begin{array}{l}\text { Partiell intra- } \\
\text { korporal }(281 \mathrm{~g})\end{array}$ & $\begin{array}{l}\text { Kontinuierlich (bis } \\
10 \mathrm{l} / \mathrm{min} \text { ) }\end{array}$ & 6000 bis $15.000 \mathrm{U} / \mathrm{min}$ \\
\hline $\begin{array}{l}\text { VAD Heart Ware }{ }^{\circledR} \\
\left(\text { Heart Ware }^{\circledR}\right)\end{array}$ & $\begin{array}{l}\text { Zentrifugal- } \\
\text { pumpe Axial- } \\
\text { rotationspumpe } \\
\text { (elektrisch) }\end{array}$ & $\begin{array}{l}\text { Mittel- bis lang- } \\
\text { fristig (komplett) }\end{array}$ & $\begin{array}{l}\text { Partiell intra- } \\
\text { korporal }(160 \mathrm{~g})\end{array}$ & $\begin{array}{l}\text { Kontinuierlich (bis } \\
101 / \mathrm{min} \text { ) }\end{array}$ & 1800 bis $4000 \mathrm{U} / \mathrm{min}$ \\
\hline
\end{tabular}




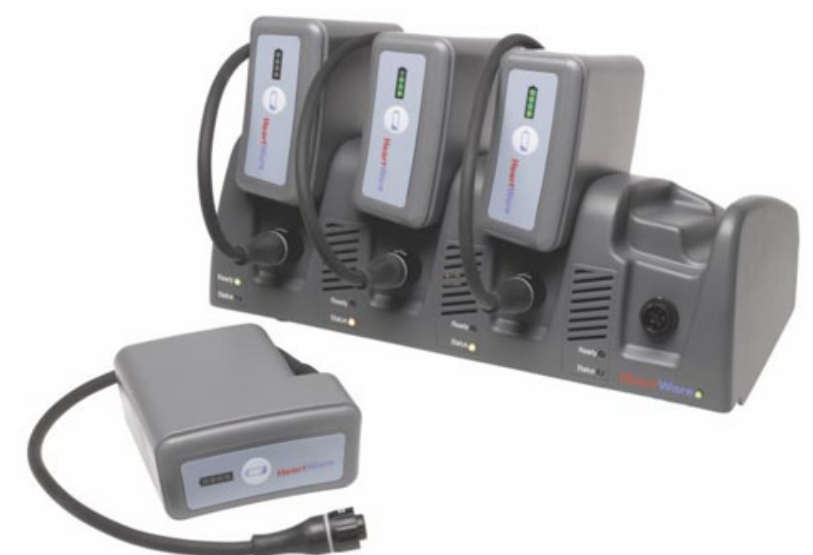

Abb. 4 Ladestation für Akkumulatoren des HeartWare $^{\circledR}$ Systems [33]

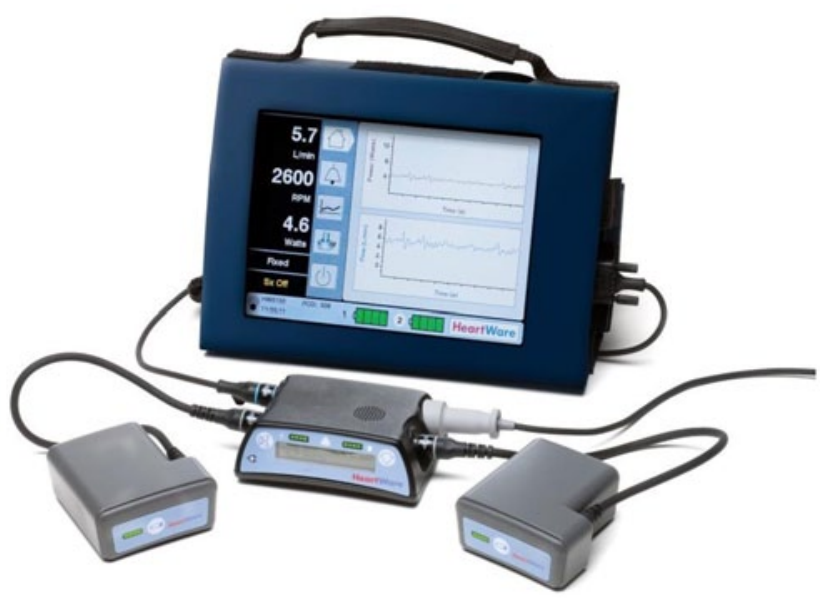

Abb. 5 Controller, Monitor und Akkumulatoren des HeartWare $^{\circledR}$-Systems [34]

Idealerweise sollte die Einstellung der Pumpengeschwindigkeit bereits im Operationssaal bei noch liegender Sonde der transösophagealen Echokardiographie erfolgen. Die Kreislaufverhältnisse des Patienten sollten zu diesem Zeitpunkt bereits stabil sein.

Die echokardiografischen und hämodynamischen Befunde sollten dann auf der Intensivstation und vor Verlegung bzw. bei Verdacht auf unzureichende Kreislaufunterstützung noch einmal wiederholt werden. Sind auch diese Kontrollen unauffällig, so bleibt die initial eingestellte Umdrehungszahl unverändert.

Ein für den Rehabilitationsmediziner besonders interessanter Aspekt ist das hämodynamische Verhalten des Patienten bei fixer bzw. steigender Umdrehungszahl unter körperlicher Belastung (z. B. im Rahmen einer Spiroergometrie). Während bei den älteren pulsatilen Unterstützungssystemen eine Anpassung des Pumpenflusses über eine Erhöhung der Herzfrequenz unter Belastung erfolgte, bleibt der Pumpenfluss bei den Continuous-Flow-Pumpen bei fix eingestellter Umdrehungszahl zunächst konstant. Durch die Erhöhung des Drucks im linken Ventrikel sinkt das Druckgefälle hin zur Peripherie und es kommt zu einem gewissen Anstieg des Pumpenflusses, der letztendlich dann wiederum durch den Durchmesser der Pumpe limitiert wird. Die bei einer Belastung auftretende Katecholaminausschüttung bewirkt eine Steigerung der kardialen Pumpleistung bei noch verbliebener Restfunktion des linken Ventrikels, sodass die systemische Perfusion während der Belastung durch zwei parallel geschaltete Pumpen (linker Ventrikel und LVAD mit fixer Umdrehungszahl) generiert wird. Trotzdem weisen LVAD-Patienten in der Regel eine deutlich eingeschränkte Leistungskapazität während körperlicher Belastung auf. Daher wurde in jüngeren Arbeiten untersucht, ob sich die Leistungsparameter bei LVAD-Patienten verbessern lassen, wenn unter Belastung die Umdrehungszahl der Pumpe erhöht wird. So konnte zum Beispiel der Peak $\mathrm{VO}_{2}$ bei Patienten mit guter Funktion des rechten Ventrikels unter Belastung durch Erhöhung der LVAD-Umdrehungszahl gesteigert werden [36]. Hier sind jedoch weitere Studien notwendig, um die Leistungsfähigkeit von LVAD-Patienten unter Belastungsbedingungen zu verbessern.

Die Flussrate, die am Monitor abzulesen ist, wird aus der Umdrehungszahl, dem Energieverbrauch und der Viskosität des Blutes, gemessen am Hämatokritwert des Patienten, ermittelt. Es handelt sich dabei nur um einen Trend, nicht aber um einen präzisen Wert, sodass hierbei vor allem Abweichungen von den sonst erhobenen Werten aufmerksam machen sollten.

Der mittlere arterielle Blutdruck soll auf jeden Fall zwischen 70 und $80 \mathrm{mmHg}$ gehalten werden. Ein mittlerer arterieller Blutdruck von $>90 \mathrm{mmHg}$ geht mit einem erhöhten Risiko für hämorrhagische Insulte einher [37].

Alarme

Optische und akustische Alarme geben dem Arzt und dem Patienten Aufschluss über den Zustand von Pumpe, Steuereinheit, Anschlüssen und Stromversorgung (Akkus, ACAdapter, DC-Adapter). Alarmzustände sind nach hoher, mittlerer und niedriger Dringlichkeit klassifiziert. Die einzelnen Alarme unterscheiden sich durch 1) einen bestimmten Signalton, 2) eine visuelle Anzeige (rot blinkend, gelb blinkend oder gelb) und 3) eine Meldung.

Ein Alarm hoher Dringlichkeit ist der lauteste Alarm. Die Alarmanzeige an der Steuereinheit blinkt rot, und die Textmeldung fordert zur unverzüglichen Reaktion auf, da ein Pumpenstillstand, eine Störung der Steuereinheit oder eine unzureichende Stromversorgung für den Betrieb der Pumpe vorliegt. Auf dem Monitor werden ebenfalls Alarminformationen angezeigt. 
Die Alarme werden im Folgenden am Beispiel des HeartWare ${ }^{\circledR}$-Systems besprochen. Die Alarmkonfigurationen sind aber auch beim HeartMate II $^{\circledR}$-System ähnlich.

Dabei können folgende Alarme hoher Dringlichkeit auftreten:

Keine Stromquelle Wenn beide Stromquellen von der Steuereinheit getrennt werden, ertönt ein lauter, kontinuierlicher Alarm, auf dem Display der Steuereinheit wird jedoch keine Meldung angezeigt. Die Pumpe läuft nicht, und die Stromquellen müssen unverzüglich angeschlossen werden. Wenn der Alarmzustand durch diese Aktion nicht behoben wird, sollte die Steuereinheit ausgetauscht werden.

VAD gestoppt Die Pumpe wird abgestellt, wenn das Verbindungskabel getrennt wurde oder wenn die Steuereinheit ausfällt. Die Textmeldung gibt an, ob das Verbindungskabel angeschlossen oder die Steuereinheit ausgetauscht werden muss.

Steuereinheit defekt Dieser Alarm weist auf einen möglichen Ausfall der Steuereinheit hin, und die Steuereinheit sollte durch eine neue Einheit ersetzt werden. Die Pumpe pumpt möglicherweise nicht.

Der Alarm mittlerer Dringlichkeit beginnt mit niedriger Lautstärke, die im Laufe der nächsten Minute zunimmt, sofern die Taste zum Stummschalten des Alarms nicht gedrückt wird. Alarme mittlerer und niedriger Dringlichkeit werden durch Drücken der Taste zum Stummschalten des Alarms für 5 min stummgeschaltet bzw. bis ein weiterer Alarm auftritt. Wenn die Taste „Alarm stummschalten“ nach Ablauf der 5 min nicht gedrückt wurde, wird die Lautstärke des Alarms auf die Lautstärke für Alarme hoher Dringlichkeit erhöht. Ein Alarm mittlerer Dringlichkeit wird durch eine blinkende gelbe Alarmanzeige gemeldet, und die Textmeldung fordert den Patienten dazu auf, eine medizinische Fachkraft zu rufen, um Anweisungen für die Behebung des Alarmzustands zu erhalten.

Folgende Alarme mittlerer Dringlichkeit können auftreten:

Hohe Leistung Dieser Alarm warnt vor einer zu hohen Leistung beim Betrieb der Pumpe. Der Alarm wird ausgelöst, wenn die Leistung (Watt) den Grenzwert des Alarms „Hohe Leistung“ überschreitet. Dies kann ein Hinweis auf eine Thrombusbildung innerhalb der Pumpe sein.

Elektrikfehler Dieser Alarm wird durch einen Fehler im Schaltkreis zwischen Pumpe und Steuereinheit ausgelöst. Der Fehler kann durch den Pumpenmotor, Verbindungskabel und -anschluss oder die Steuereinheit verursacht werden. Das Audiosignal dieses Alarms kann über den Monitor permanent deaktiviert werden.

Fluss niedrig Der Alarm „Fluss niedrig“ wird ausgelöst, wenn der durchschnittliche Durchfluss unter den Grenzwert des Alarms „Fluss niedrig“ abfällt.

Ein Alarm niedriger Dringlichkeit wird durch eine gelbe Alarmanzeige gemeldet. Die Meldung gibt an, ob ein schwacher Akku ausgetauscht oder ob das System wieder an eine Stromquelle (Akku, AC- oder DC-Adapter) angeschlossen werden muss. Der Alarm wird nach 5 min und dann nach 10 min erneut lauter, wenn der Alarm nicht stummgeschaltet wird.

Folgende Alarme niedriger Dringlichkeit können auftreten:

Akkumulator niedrig Dieser Alarm wird ausgelöst, wenn ein Akkumulator nur noch eine Restkapazität zwischen 10 und $25 \%$ aufweist.

Stromquelle getrennt Dieser Alarm wird ausgelöst, wenn eine an der Steuereinheit angeschlossene Stromquelle getrennt wird oder nicht richtig funktioniert. Die Stromquelle sollte unverzüglich wieder angeschlossen werden, da der Patient sonst über keine Reservestromquelle verfügt [26].

\section{Unterdruckzustand}

$\mathrm{Zu}$ einem Unterdruckzustand kann es bei einem Ventrikelkollaps oder einer Verlegung des Einflusswegs kommen. Ein Ventrikelkollaps tritt auf, wenn ein VAD mit kontinuierlichem Durchfluss versucht, mehr Blut vom linken Ventrikel zu fördern, als zur Verfügung steht. Dies resultiert in einer beträchtlichen Reduzierung des Ventrikelvolumens. Ein Kollaps des linken Ventrikels kann das Ergebnis klinischer Ereignisse sein, die die linksventrikuläre Vorlast beeinflussen. Dazu gehören Hypovolämie (z. B. Blutung, aber auch zu starke Dehydrierung), Rechtsherzinsuffizienz, Arrhythmien und Lungenembolien. Eine Verstopfung des Einflusswegs tritt auf, wenn die Einflusskanüle durch das interventrikuläre Septum blockiert wird und dadurch einen Unterdruckzustand verursacht. Eine temporäre Verstopfung des Einflusswegs kann als Ergebnis der chirurgischen Positionierung, der Patientenlage oder bei Überanstrengung (Valsalva-Manöver) auftreten.

Der VAD-Träger sollte in der Lage sein, diesen Alarm zu beheben. Er kann diesen selbst abschalten und muss dann bei seinem behandelnden VAD-Zentrum das Ereignis melden. 


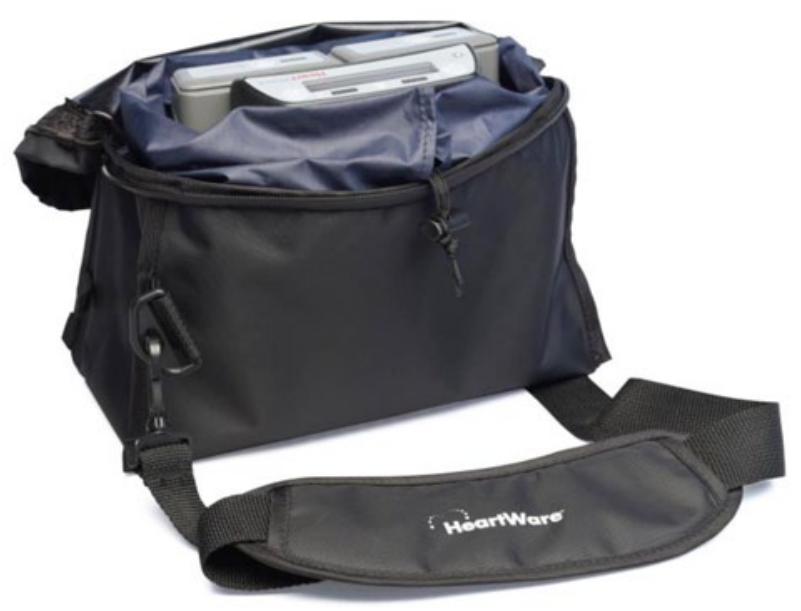

Abb. 6 Duschtasche der Firma HeartWare ${ }^{\circledR}$ zum Schutz des VADs vor Wasserkontakt [33]

\section{Empfehlungen für den Alltag}

Der Patient sollte stets eine Reserve-Steuereinheit und vollständig aufgeladene Akkus bereithalten, um auf Störungen und Ausfälle der Steuereinheit vorbereitet zu sein und entsprechend reagieren zu können. Auf der ReserveSteuereinheit sollten die gleichen Pumpenparameter und Patientendaten eingestellt sein wie auf der zur Unterstützung verwendeten Steuereinheit. Ein Ausfall der Steuereinheit bzw. eine Störung der Steuereinheit erzeugen einen Alarm hoher Dringlichkeit, der in Rot angezeigt wird und auf dem Display der Steuereinheit erscheint.

Die Akkumulatoren enthalten Lithium-Ionen-Zellen, mit denen das LVAD ca. 4 bis 6 h betrieben werden kann. Die Kapazität jedes Akkumulators in Stunden basiert auf:

- Energieverbrauch von Steuereinheit und Pumpe während des Betriebs,

- Anzahl der Lade- und Entladezyklen des Akkumulators.

Die erwartete Nutzungsdauer der Akkumulatoren beträgt mehr als 500 Lade- und Entladezyklen. Wenn ein Akkumulator nur noch $2 \mathrm{~h}$ Unterstützungsbetrieb speisen kann, sollte er ausgetauscht werden.

Ein zeitweiser Betrieb der Akkumulatoren bei Temperaturen unter $0^{\circ} \mathrm{C}$ reduziert zwar ihre Kapazität, die Funktion bleibt jedoch normal.

Die Langzeitlagerung der Akkumulatoren außerhalb eines Bereichs von -20 bis $+25^{\circ} \mathrm{C}$ kann ihre Kapazität dauerhaft reduzieren. Die Akkumulatoren sollten daher am besten bei Zimmertemperatur gelagert werden.

Systemkomponenten dürfen nicht in Flüssigkeiten eingetaucht werden. Duschen und Waschen ist zwar erlaubt, sobald der Arzt bescheinigt, dass die Wunde ausreichend verheilt ist. Zum Duschen muss jedoch ein spezielles Duschkit mit einer Duschtasche verwendet werden (Abb. 6).
Wenn das Ladegerät mit Wasser/Feuchtigkeit, Regen/ Schnee oder feuchten Oberflächen in Kontakt kommt, kann ein schwerer Stromschlag bzw. eine Funktionsstörung des Ladegeräts die Folge sein.

Zur Steigerung der Mobilität des Patienten können die Steuereinheit und zwei Akkumulatoren in einer Patiententasche sicher verstaut und transportiert werden.

Ausblick Zurzeit sind Multizenterstudien von Thoratec ${ }^{\circledR}$ mit dem HeartMate $3{ }^{\circledR}$ und von HeartWare ${ }^{\circledR}$ mit dem MVADSystem gestartet. Das HeartMate $3^{\circledR}$-LVAD ist eine neue kompakte intraperikardiale Zentrifugalpumpe mit einem magnetisch aufgehängten Rotor und einem artifiziellen Puls (30/min), um der Bildung von Gerinnseln vorzubeugen.

Das MVAD ist kleiner als sein Vorgänger und ist damit leichter minimal-invasiv zu implantieren. Vollimplantierbare Systeme mit kabellosem transkutanem Energietransfer (TET-Systeme) stehen bislang nur für tierexperimentelle Studien zur Verfügung.

\subsection{Kardiologische Diagnostik während der Rehabilitation von VAD-Patienten}

Die diagnostischen Maßnahmen bei Herzunterstützungssystemen (VAD) haben im Rahmen einer Rehabilitation folgende Zielsetzungen:

- Erkennen einer Dysfunktion des VAD primär mittels Echokardiographie (z. B. Hypovolämie, Thrombosierung)

- Überprüfung von Nierenfunktion, Elektrolythaushalt, Blutbild, Entzündungszeichen und Antikoagulation durch Laboruntersuchungen (siehe Abschn. 2.14)

- Erkennen von Grundrhythmus und Rhythmusstörungen durch Ruhe-, Belastungs-, Langzeit-EKG und - falls vorhanden -Kontrolle des Schrittmachers/ICD (siehe Abschn. 2.6)

- Überprüfung der korrekten Lage des VAD und anderer Devices sowie Diagnose einer Überwässerung und pulmonaler Infektionen (Röntgen-Thorax)

- Optimierung einer medikamentösen Herzinsuffizienztherapie mit dem Ziel eines niedrigen arteriellen Mitteldrucks $<80 \mathrm{mmHg}$ (konventionelle oder DopplerBlutdruckmessung, Langzeit-Blutdruck) und damit Unterstützung der Myokarderholung

- Erkennen schlafbezogener Atmungsstörungen (Schlafapnoe-Screening)

Die Echokardiografie sollte in erweiterter Form nach den Vorgaben der Fachgesellschaften erfolgen [38-41]. Für die Besonderheiten von Untersuchungen bei VAD liegen Empfehlungen auf der Basis von Expertenmeinungen vor [42-45]. Aktuell publiziert wurden Guidelines der American Society of Echocardiography [46]. Durch die apikale Lage 
des Devices, die Driveline mit umgebendem Verband und bedingt durch Vernarbungen ist die Echoqualität vom apikalen und meist auch vom subkostalen Schallfenster deutlich eingeschränkt. Weiterhin überlagert das Pumpengeräusch den Farbdoppler, sodass Vitien eingeschränkt zu quantifizieren sind. Die Optimierung der Pumpengeschwindigkeit nach Echokriterien erfolgt üblicherweise im implantierenden Zentrum.

Ziele der echokardiographischen Diagnostik im Rahmen einer Rehabilitation sind [13]:

- Bestätigung einer normalen VAD-Funktion

- Ursachenklärung bei VAD-Dysfunktion, Komplikationen oder klinischen Zeichen einer Herzinsuffizienz

- Bestimmung des Flüssigkeitshaushalts (Hyper-, Hypovolämie)

- Erkennung einer Erholung der linksventrikulären Funktion (recovery)

Der Echobefund sollte neben den Messwerten einer BasisEchokardiographie folgende spezielle Befunde beschreiben [46]:

- Öffnung der Aortenklappe (Häufigkeit, Dauer und Ausmaß). Das Vorhandensein von Thromben im Bulbus aortae, insb. bei nicht öffnender $\mathrm{AK}$, und gegebenenfalls das Ausmaß einer vorhandenen Aortenklappenregurgitation

- Beschreibung von intrakavitären Thromben insbesondere an der proximalen Kanüle und von Klappenauflagerungen

- Stellung des interventrikulären Septums zur InflowKanüle und Beschreibung des Bewegungsmusters des interatrialen und -ventrikulären Septums

- Flussgeschwindigkeiten und Pulsatilität an der InflowKanüle und im Outflow-Graft

- Größe und Funktion des linken und des rechten Ventrikels

- Ausmaß einer Mitral- und TrikuspidalklappenRegurgitation und Bestimmung des pulmonalarteriellen Drucks

- Diameter mit Atemmodulation der Vena cava inferior

- Vorhandensein eines Perikard- oder Pleuraergusses und Prüfung der Zwerchfellbeweglichkeit

- Darstellung der Driveline insb. bei Entzündungszeichen

Die Laboruntersuchungen sollten sich an den häufigsten Komplikationen (Blutungen, Thrombosierungen, Infektionen, Elektrolytstörungen) orientieren. Parameter der Hämolyse sind als Zeichen einer Thrombosierung essenziell, Biomarker optional, da die Wertigkeit als therapeutisches Steuerungsinstrument nicht untersucht ist. Eine Verlaufskontrolle von NT-proBNP kann jedoch sinnvoll sein. Bei Infektionszeichen sollte auf die frühe Abnahme von mikrobiologischen Untersuchungen (Abstriche, Blutkulturen) geachtet werden. Das Monitoring der Blutgerinnung wird in einem eigenen Abschnitt besprochen Verweis auf 2.14
Schulung Die INR-Bestimmung sollte mind. $2 \times /$ Woche erfolgen, die Häufigkeit richtet sich nach den Vorgaben des implantierenden Zentrums. Sie kann u. U. auch täglich erforderlich sein.

Ruhe-, Belastungs- und Langzeit-EKGs dienen dem Erkennen des Grundrhythmus und von proarrhythmischen Effekten der Medikation (QT-Zeit) und häufig auftretenden malignen Herzrhythmusstörungen (ventrikulären Tachykardien) sowie der aktuellen Belastbarkeit und werden in üblicher Form durchgeführt. Zur Wertigkeit der Spiroergometrie und des 6-Minuten-Gehtests siehe Abschn. 2.5.

Die Röntgenuntersuchung des Thorax ist optional und dient, neben dem Erkennen einer pulmonalvenösen Stauung, eines Pleuraergusses oder einer Pneumonie, der Lagekontrolle des VAD oder anderer Devices wie ICD-, CRT- oder CCM-System.

Eine hohe Bedeutung kommt der Messung des arteriellen Blutdrucks zu. Ein Hochdruck kann nicht nur die Funktion des VAD durch Erniedrigung des Flusses und der ventrikulären Entlastung verschlechtern, sondern auch die Komplikationsrate insbesondere für intrazerebrale Blutungen erhöhen [13].

Bedingt durch den kontinuierlichen Fluss der modernen VADs (nichtpulsatile Systeme) ist der Blutdruck jedoch mit den konventionellen Blutdruckmessgeräten auf auskultatorischer und oszillometrischer Basis oft nicht durchführbar. Die Messung muss dann mit der Dopplermethode erfolgen, und das Ergebnis entspricht einem Wert zwischen arteriellem Mitteldruck und systolischem Blutdruck [47]. Nach Expertenmeinung ohne Nachweis durch Studien wird ein mittlerer Blutdruck von $\leq 80 \mathrm{mmHg}$ angestrebt [13]. Spezielle Blutdruckmessgeräte mit besserer Messgenauigkeit bei nichtpulsatilen Systemen sind in Erprobung [47, 48].

Bei Herzinsuffizienz-Patienten werden schlafbezogene Atmungsstörungen häufig gefunden, und ihre Diagnostik wird empfohlen [49]. Für Patienten mit VAD liegen jedoch keine Daten vor, Studien zur klinischen Bedeutung der Schlafapnoe werden aktuell durchgeführt.

In Tab. 3 sind einige spezielle echokardiographische Messparameter bei VAD-Patienten zusammengefasst und nach der MoSCoW-Priorisierung geordnet (Expertenkonsens). Empfohlener Messzeitpunkt ist der Beginn der Rehabilitation und bei Bedarf.

\section{Monitoring}

In der Literatur finden sich keine Angaben über routinemäßiges Monitoring nach Aufnahme in der kardiologischen Rehabilitation. Folgende Techniken werden angewendet: Telemetrie, kontinuierliches EKG, Sauerstoffsättigung über Fingerclip. Für den Überwachungsbedarf eines VAD- 
Tab. 3 Spezielle echokardiografische Messparameter bei VAD-Patienten bei Beginn der Rehabilitation und bei Bedarf (modifiziert nach [46])

\begin{tabular}{|c|c|c|}
\hline Ort & Parameter & MoSCoW \\
\hline Inflow-Kanüle & $\begin{array}{l}\text { Position, Flussmuster und -geschwindigkeit ( } \mathrm{min} / \mathrm{max} \text {, abhängig vom VAD, typisch } 2 \mathrm{~m} / \mathrm{s} \text { ), } \\
\text { Thromben }\end{array}$ & MUST \\
\hline Outflow-Graft & Flussmuster und -geschwindigkeit (min/max Normwert 1-2 m/s) & MUST \\
\hline Aortenklappe & Öffnung (Vollständigkeit, Häufigkeit), Graduierung Insuffizienz & MUST \\
\hline Mitralklappe & Graduierung Insuffizienz & MUST \\
\hline Trikuspidalklappe & $\begin{array}{l}\text { Systolische Bewegung oder Gewebegeschwindigkeit des Anulus, Flussgeschwindigkeit der } \\
\text { Regurgitation zur Abschätzung des pulmonalarteriellen Drucks }\end{array}$ & MUST \\
\hline Interventrikuläres Septum & Stellung und Bewegungsmuster während des Herzzyklus (Synchronie) & MUST \\
\hline Interatriales Septum & Beschreibung eines persistierenden Foramen ovale, gegebenenfalls des Shuntvolumens & MUST \\
\hline Vena cava inferior & Diameter mit Atemmodulation & MUST \\
\hline $\begin{array}{l}\text { Aortenwurzel und } \\
\text { aszendierende Aorta }\end{array}$ & Thromben, Diameter & MUST \\
\hline Perikard & Erguss & MUST \\
\hline Pleura & Erguss, Höhe und Beweglichkeit der Zwerchfelle & MUST \\
\hline Linker Ventrikel & 2D-Strain-Analyse (Globaler longitudinaler oder radialer Strain) & COULD \\
\hline Outflow-Kanüle & Messung des Herzminutenvolumens über Doppler & COULD \\
\hline RV-Ausfluss-Trakt & Messung des Herzminutenvolumens über Doppler & COULD \\
\hline
\end{tabular}

Patienten in der Rehabilitation sind folgende Aspekte wichtig:

- Generell entscheidet die zuweisende Klinik, ob ein Patient mit einem VAD-System in der Rehabilitationsklinik behandelt werden kann. Bei kontinuierlichem Überwachungsbedarf ist in der Regel die Rehabilitationsfähigkeit nicht gegeben.

- Das VAD-System stellt ein patientenbezogenes Device dar. Der Patient ist in die Bedienung des Geräts bereits vor der Rehabilitation eingewiesen worden und sollte sie selbstständig beherrschen. Bei diesem Lernprozess kann die Rehabilitation Unterstützung bieten.

- Rehabilitation stellt eine Übergangsphase in das häusliche Umfeld dar, in der der Patient ohne Überwachung auskommen muss.

\subsection{Leistungsdiagnostik nach VAD-Implantation}

Voraussetzung für eine effektive und sichere Durchführung einer Bewegungstherapie im Rahmen der Rehabilitation ist eine umfassende Risikostratifizierung inklusive einer Leistungsdiagnostik. Diese dient primär der Einschätzung des kardiovaskulären Gefährdungspotenzials des Patienten im Hinblick auf die hämodynamische Belastungsreaktion (adäquate Herzfrequenz-, Blutdruckreaktion), den Myokardfaktor (Ventrikelgeometrie, systolische/diastolische Funktion, Narben, Dystrophien), den Rhythmusfaktor (Regelmäßigkeit, tachy-/bradykard), den Ischämiefaktor (Koronarfaktor, Zustand Herzkranzgefäße), die Atemeffizienz (ventilatorische Antwort auf körperliche Belastung), sowie einen ggf. vorhandenen pulmonalen Hochdruck. Hierbei addieren sich Effekte des nativen Herzens mit denen des mechanischen Unterstützungssystems. Zudem müssen leistungslimitierende Komorbiditäten, insbesondere im internistischen, neurologischen und orthopädischen Bereich, erfasst und dokumentiert werden. Dies ermöglicht eine Einordnung des Patienten zu einer Risikoklasse hinsichtlich seines Gefährdungspotenzials, die bei der Planung, Durchführung und Überwachung der Rehabilitationsmaßnahme und im Rahmen der Bewegungstherapie Berücksichtigung finden muss.

Die Leistungsdiagnostik erlaubt die Ermittlung der kardiopulmonalen, metabolischen und muskulären Belastungstoleranz des Patienten. Dabei sollten die objektive Belastbarkeit durch standardisierte Testverfahren, aber auch die subjektive Wahrnehmung des Patienten ermittelt werden. Bei diesen schwer kranken Patienten, die nicht selten eine lange Krankheitsgeschichte mit zum Teil wiederholten Immobilisationsphasen aufweisen, ist die Erfassung der Eigenwahrnehmung des Patienten, seiner Leistungsbereitschaft und Selbsteinschätzung essenziell. Es werden hierdurch sowohl Leistungspotenziale als auch Defizite des Patienten ermittelt und deren Konsequenzen für die Alltagsbelastbarkeit, Mobilität, Aktivität und Teilhabe abgeleitet. Diese wiederum beeinflussen Zielsetzung, Planung und Durchführung der Bewegungstherapie.

Des Weiteren kann die Leistungsdiagnostik zur Überprüfung der Effizienz der medikamentösen Einstellung und anderer therapeutischer Maßnahmen sowie zur Einschätzung der Prognose dienen.

Bei der Durchführung aller leistungsdiagnostischen Maßnahmen müssen die besonderen Merkmale und Limitierungen, die durch das VAD gegeben sind, berücksichtigt und spezielle Sicherheitsstandards eingehalten werden. Dies setzt voraus, dass die Leistungsdiagnostik bei diesem Patientenkollektiv ausschließlich von speziell geschultem Personal durchgeführt wird. Bei allen leistungs- 
diagnostischen Maßnahmen müssen Pumpenfluss und Leistungsanzeige beachtet werden. Zudem muss gewährleistet sein, dass die Funktion des VAD nicht durch die diagnostischen Maßnahmen beeinträchtigt wird. Dies betrifft die Platzierung der Tasche mit dem extrakorporalen Equipment (Steuerungseinheit und Akkumulatoren), die sicher, körpernah und ohne Driveline-Belastung (Zugbelastung, Erschütterungen, Knicken) untergebracht und befestigt werden muss (Safarijacke oder Gürteltasche, ggf. spezielle Haltekörbchen/-ständer für Tragetaschen). Für VAD-Träger sind bei allen Testverfahren zudem systemrelevante Abbruchkriterien zu beachten. Diese sind der Systemalarm, der Pumpenfluss $<31$ und Blutungen (z. B. Nasenbluten) [13, 50]. Bei Vorliegen einer pulmonalen Komorbidität sollte zudem die Sauerstoffsättigung mit einem Pulsoxymeter gemessen werden und ein Abbruch bei Werten $<90 \%$ erfolgen [50].

Des Weiteren gelten die üblichen Abbruchkriterien für Belastungsuntersuchungen [51]. Relevante Kontraindikationen der Leistungsdiagnostik speziell für dieses Patientenkollektiv sind nicht publiziert. Es gelten die für Herzinsuffizienzpatienten publizierten Kontraindikationen [52].
VAD-Patienten sind ein sehr heterogenes Patientenkollektiv hinsichtlich des Krankheitsverlaufs und der daraus resultierenden kardiopulmonalen und muskulären Leistungseinschränkung [53]. Dies erschwert die Formulierung genereller Empfehlungen zur Leistungsdiagnostik für diese Patientengruppe. Derzeit gibt es für VAD-Träger keine einheitliche internationale Leitlinie zur Leistungsdiagnostik; alle bis jetzt formulierten Empfehlungen sind sehr vage und basieren auf Erfahrungen weniger Untersucher mit kleineren Patientengruppen [13, 53].

In Tab. 4 sind mögliche Assessmentverfahren für diese Patientengruppe zusammengefasst, wobei die individuelle Situation des Patienten ausschlaggebend für die Anwendung ist.

\subsection{Umgang mit Herzrhythmusstörungen sowie mit ICD-, CRT- und Herzschrittmacheraggregaten während der Rehabilitation von VAD-Patienten}

Im Rahmen der Rehabilitation von VAD-Patienten treten häufig Herzrhythmusstörungen bzw. Fragen bezüglich zusätzlich implantierter Aggregate (ICD, CRT-D, CCM) auf.

Tab. 4 Mögliche Assessments der Leistungsdiagnostik für Patienten mit einem Herzunterstützungssystem (VAD)

\begin{tabular}{|c|c|c|c|c|c|c|}
\hline & Test & Testprotokoll & Messparameter & Testzeitpunkte & Testleiter $^{\mathrm{a}}$ & MoSCoW \\
\hline \multirow[t]{2}{*}{$\begin{array}{l}\text { Funktionelles } \\
\text { Assessment }\end{array}$} & 6-Minuten-Gehtest & $\begin{array}{l}\text { ATS-Standard } \\
{[54] \text { mit/ohne }} \\
\text { Hilfsmittel }\end{array}$ & $\begin{array}{l}\text { Strecke }(\mathrm{m}), \text { mittl. } \\
\text { Geschw. }(\mathrm{km} / \mathrm{h}) \text {, } \\
\text { Pausenanzahl/-dauer, } \\
\text { Abbruchgrund, RPE }\end{array}$ & $\begin{array}{l}\text { Rehabeginn } \\
( \pm 3-7 \text { Tage }) \\
\text { Rehaende, bei } \\
\text { Bedarf }\end{array}$ & $\begin{array}{l}\text { Examinierter } \\
\text { Therapeut, Pflegekraft } \\
\text { oder Arzthelfer }\end{array}$ & MUST \\
\hline & $\begin{array}{l}\text { Timed ,Up and } \\
\text { Go"-Test }\end{array}$ & $\begin{array}{l}\text { Standard [55] mit/ } \\
\text { ohne Hilfsmittel }\end{array}$ & $\begin{array}{l}\text { Zeit (Sek.), Be- } \\
\text { wegungssicherheit, } \\
\text { Klassifikation der } \\
\text { Mobilität }\end{array}$ & $\begin{array}{l}\text { Rehabeginn } \\
( \pm 3-7 \text { Tage }) \\
\text { Rehaende }\end{array}$ & $\begin{array}{l}\text { Examinierter } \\
\text { Therapeut, Pflegekraft } \\
\text { oder Arzthelfer }\end{array}$ & SHOULD $^{b}$ \\
\hline \multirow[t]{3}{*}{ Ergometrie } & $\begin{array}{l}\text { Fahrrad ohne } \\
\text { Spiroegometrie }\end{array}$ & $\begin{array}{l}\text { 1. Rampe } \\
(5-10 \mathrm{~W} / \mathrm{min})[56] \\
\text { 2. WHO [51] }\end{array}$ & $\begin{array}{l}\text { EKG, HF, RPE, Watt, } \\
\text { Abbruchgrund }\end{array}$ & $\begin{array}{l}\text { Rehabeginn } \\
( \pm 3-7 \text { Tage }) \\
\text { Rehaende }\end{array}$ & $\begin{array}{l}\text { Arzt und examinierter } \\
\text { Therapeut, Pflegekraft } \\
\text { oder Arzthelfer }\end{array}$ & SHOULD \\
\hline & $\begin{array}{l}\text { Fahrrad mit } \\
\text { Spiroergometrie }\end{array}$ & $\begin{array}{l}\text { 1. Rampe } \\
(5-10 \mathrm{~W} / \mathrm{min})[56] \\
\text { 2. WHO [51] }\end{array}$ & $\begin{array}{l}\text { EKG, HF, RPE, } \\
\text { Watt }_{\text {max }}, \text { Abbruchgrund, } \\
\mathrm{VO}_{2 \text { peak }}, \mathrm{VO}_{2} \mathrm{R}, \mathrm{VAT}, \\
\mathrm{VE}^{\mathrm{C}} \mathrm{CO}_{2}-\mathrm{Slope}, \mathrm{RQ}\end{array}$ & $\begin{array}{l}\text { Rehabeginn } \\
( \pm 3-7 \text { Tage }) \\
\text { Rehaende }\end{array}$ & $\begin{array}{l}\text { Arzt und examinierter } \\
\text { Therapeut, Pflegekraft } \\
\text { oder Arzthelfer }\end{array}$ & SHOULD \\
\hline & $\begin{array}{l}\text { Laufband }{ }^{c} \\
\text { mit/ohne } \\
\text { Spiroergometrie }\end{array}$ & $\begin{array}{l}\text { Protokoll aus- } \\
\text { wählen mit } \\
\text { konstant niedriger } \\
\text { Geschw. und } \\
\text { Lastanstieg durch } \\
\text { Steigungsanstieg }\end{array}$ & $\begin{array}{l}\text { EKG, HF, RPE, Ab- } \\
\text { bruchgrund/ } \mathrm{VO}_{2 \text { peak }} \text {, } \\
\mathrm{VO}_{2} \mathrm{R} \text { VAT, } \mathrm{VE}^{-\mathrm{CO}_{2}-} \\
\text { Slope, RQ }\end{array}$ & $\begin{array}{l}\text { Rehabeginn } \\
\text { ( } \pm 3-7 \text { Tage }) \\
\text { Rehaende }\end{array}$ & $\begin{array}{l}\text { Arzt und examinierter } \\
\text { Therapeut, Pflegekraft } \\
\text { oder Arzthelfer }\end{array}$ & COULD \\
\hline \multirow[t]{2}{*}{$\begin{array}{l}\text { Kraft- } \\
\text { Assessment }\end{array}$} & „Handgrip““ & $\begin{array}{l}\text { Standard [57], } \\
\text { einarmig }\end{array}$ & $\begin{array}{l}\text { Newton }_{\text {max }} \text {, rechte und } \\
\text { linke Hand }\end{array}$ & $\begin{array}{l}\text { Rehabeginn } \\
( \pm 3-7 \text { Tage }) \\
\text { Rehaende }\end{array}$ & $\begin{array}{l}\text { Examinierter } \\
\text { Therapeut }\end{array}$ & COULD \\
\hline & Knie-Extension & $\begin{array}{l}\text { Standard [58], } \\
\text { einbeinig }\end{array}$ & $\begin{array}{l}\text { Newton }_{\text {max }} \text {, rechtes und } \\
\text { linkes Bein }\end{array}$ & $\begin{array}{l}\text { Rehabeginn } \\
\text { ( } \pm 3-7 \text { Tage }) \\
\text { Rehaende }\end{array}$ & $\begin{array}{l}\text { Examinierter } \\
\text { Therapeut, Pflegekraft }\end{array}$ & COULD \\
\hline
\end{tabular}

${ }^{\text {aVAD-geschultes Personal }}$

beei einer 6-Minuten-Gehstrecke unter 200 m und bei Patienten mit Rollator als Gehhilfe

${ }^{c}$ nur bei Patienten ohne Gleichgewichtsprobleme (erhöhte Sturzgefahr), Examinierter Therapeut = Physio-, Sport- und/oder Ergotherapeut 
Herzrhythmusstörungen werden von VAD-Patienten häufig nicht bemerkt. Selbst ventrikuläre Tachykardien, vereinzelt auch Kammerflimmern, äußern sich insbesondere im Falle einer fehlenden pulmonalen Hypertonie bei einer biventrikulären Unterstützung oft nur in geringen Beschwerden, da das Unterstützungssystem die Perfusion aufrechterhält [59-61]. Problematisch ist hier eher bei einem rein linksventrikulären Unterstützungssystem der nicht unterstützte rechte Ventrikel. Dieser kann bei einer ventrikulären Herzrhythmusstörung oder auch bei schlechter Frequenzkontrolle im Rahmen einer supraventrikulären Herzrhythmusstörung, z. B. Vorhofflimmern oder-flattern, rasch zu einer klinischen Verschlechterung durch Rechtsherzinsuffizienz beitragen. Des Weiteren können aufgrund der konsekutiven schlechteren Füllung des linken Ventrikels eine Hypotension mit Low-flow-Alarmen und/oder vermehrte Ansaugphänomene auftreten [11, 13, 61].

Bei supraventrikulären Herzrhythmusstörungen sollte je nach hämodynamischer Auswirkung wie üblich leitliniengerecht therapiert werden [13]. Ob ein paroxysmales oder persistierendes Vorhofflimmern rhythmusstabilisierend therapiert werden soll, ist während einer Rehabilitation ggf. mit dem implantierenden Zentrum zu klären. Im Falle ventrikulärer Herzrhythmusstörungen sollten reversible Ursachen wie z. B. Ansaugphänomene (können sowohl Ursache als auch Folge ventrikulärer Arrhythmien sein, s. o.), Elektrolytentgleisungen, QT-Zeit-Verlängerungen, apikale Vernarbungen um die Inflow-Kanüle des LVAD und Ischämien in Betracht gezogen werden. Ansonsten sollten supraventrikuläre und ventrikuläre Rhythmusereignisse wie auch bei Patienten ohne mechanische Kreislaufunterstützung behandelt werden (Betablocker, Antiarrhythmika, Kardioversion, Defibrillation [11], Programmierung von ICD zur Vermeidung inadäquater Schocks oder in Absprache mit einem entsprechenden Zentrum auch ablative Maßnahmen $[60,62,63])$. Eine notfallmäßige Herzdruckmassage ist bei einem implantierten VAD zwar mit einem Verletzungsrisiko verbunden, jedoch möglich, wenn notwendig $[11,60]$.

Die Mehrzahl der VAD-Patienten hat bereits im Vorfeld der Operation einen ICD oder ein CRT-D-Aggregat für eine kardiale Resynchronisationstherapie erhalten [64]. Bei Aufnahme sollten entsprechende Informationen bzgl. Modell, Programmierung, Messwerte usw. vorliegen. Herkömmliche Herzschrittmacher-Systeme mit isoliert rechtsventrikulärer Stimulation zur alleinigen antibradykarden Therapie finden sich bei Patienten mit einem VAD eher selten. Die ICD-Funktion sollte aktiviert sein (nach ISHLT-Leitlinien Empfehlungsstufe Ia [13, 59]). Die gemeinsame Therapie mit einem VAD und anderen elektrischen Aggregaten ist bis auf seltene aggregatabhängige Wechselwirkungen (wie z. B. die fehlende Telemetriemöglichkeit von Atlas-ICD der Firma St. Jude Medical $[61,65]$ in Verbindung mit einem HeartMate $\mathrm{II}^{\circledR}$-VAD) als sicher anzusehen $[66,67]$. In den
Leitlinien der ISHLT ist die routinemäßige Implantation eines ICD nach Einsetzen eines Assist Devices eine Empfehlung der Stufe IIa [13, 68, 69]. ICD-Schocks sind nach Implantation eines Assist Devices durchaus häufig, aber nicht unbedingt überlebensnotwendig [13, 69, 70]. Einige Autoren gehen von einer Lebensverlängerung durch zusätzlich zum VAD implantierten ICDs aus [71]. Andere Daten wiederum zeigen keine lebensverlängernde Wirkung eines primärprophylaktisch postoperativ nach VADOperation implantierten ICD [70]. Das Risiko ventrikulärer Arrhythmien scheint insbesondere durch bereits präoperativ vor der VAD-Operation vorliegende ventrikuläre Arrhythmien bestimmt zu sein $[66,67,72]$. Eine primärprophylaktische ICD-Implantation nach Anlage eines Assist Device wird daher nicht grundsätzlich empfohlen [72, 73].

$\mathrm{Ob}$ eine laufende kardiale Resynchronisationstherapie mit durchgehender biventrikulärer Stimulation nach Implantation eines VAD sinnvoll ist, kann nicht durch Daten belegt werden. Dieses erscheint für das Zusammenspiel von eigener Herzaktion und Unterstützungssystem im Einzelfall bedeutsam zu sein [74], ähnlich wie die Durchführung einer myokardentlastenden Medikation.

Im Rahmen der Rehabilitation von VAD-Patienten sollte eine engmaschige EKG-Kontrolle erfolgen. Insbesondere bei klinischer Verschlechterung sollte an eine rhythmogene Ursache gedacht werden.

In der Rehabilitation können frequenzstabilisierende medikamentöse Maßnahmen und ggf. eine interne oder externe elektrische Kardioversion durchgeführt werden. Letzterer Eingriff sollte nur dann durchgeführt werden, wenn ein entsprechendes intensivmedizinisches Monitoring bzw. Intensivtherapie am Hause möglich sind. Ggf. ist der Patient ansonsten an die implantierende Klinik zu verweisen. Im Falle ventrikulärer Arrhythmien ist eventuell ein sofortiges Handeln erforderlich. Dieses kann die Gabe von Antiarrhythmika (in der Regel z. B. Gabe von Amiodaron), aber auch eine Kardioversion bzw. Defibrillation bedeuten. Insbesondere bei hämodynamischer Instabilität ist der elektrischen Terminierung der zugrunde liegenden Herzrhythmusstörung der Vorzug zu geben. Im Falle eines implantierten ICD- oder CRT-D-Aggregats kann bei supraventrikulären oder auch bei langsamen außerhalb des Therapiebereichs liegenden ventrikulären Arrhythmien eine interne Kardioversion eine gute Behandlungsoption sein. Dieses setzt das Vorhandensein entsprechender Kontrollgeräte und Expertise voraus. Zudem kann hier auch die Möglichkeit der Überstimulation durch antitachykardes Pacing genutzt werden.

Zusammenfassend zeigt dies, dass die Kontroll- und Therapiemöglichkeit für Schrittmacher- und ICD-Aggregate eine sinnvolle Voraussetzung für die Rehabilitation bei den betroffenen Patienten ist. Postoperativ muss nach Implantation eines VAD eine Kontrolle der zusätzlich 
implantierten ICD-, CRT- oder Herzschrittmacheraggregate erfolgen [75]. Die alleinigen Gerätekontrollen könnten auch extern erfolgen oder mit den zuweisenden Kliniken abgesprochen werden. Kontrollgeräte und entsprechende Expertise sind aber direkt in der Rehabilitationseinrichtung sinnvoll, um ggf. schnell auf Probleme reagieren zu können und Patiententransporte zu vermeiden. Sollte die ICDFunktion präoperativ deaktiviert sein, so sollte diese im Rahmen der Rehabilitation wieder aktiviert werden. Da ventrikuläre Herzrhythmusstörungen wegen der Unterstützung des VAD länger toleriert werden, sollte die Programmierung entsprechend überdacht werden. Dabei sollte der schmerzfreien Terminierung durch antitachykardes Pacing der Vorzug gegeben werden. Die Programmierung sollte so gestaltet werden, dass inadäquate Schocks vermieden werden z. B. nach Kriterien der MADIT-RIT-Studie [76]. Auch eine deaktivierte oder aus anderen Gründen nicht laufende CRT sollte, entsprechende Reizschwellen der Sonden vorausgesetzt, wieder aktiviert werden.

Fehlfunktionen der Aggregate sind nach Implantation eines Assist Devices in der Rehabilitation durchaus häufig [75]. Oft werden diese auch erst im Rahmen der Rehabilitation erkannt (z. B. Anstiege der Reizschwellen und daran noch nicht angepasste Einstellung der Amplituden, Dislokationen von Sonden, Sensingdefekte, perioperative Programmierung hoher Stimulationsfrequenzen, VVI-Stimulation bei etabliertem Sinusrhythmus, ERI erreicht, perioperativ ausgestellte ICD- oder CRT-Funktion etc.). Nach einer eigenen Datenanalyse liegen mehr oder weniger schwerwiegende Funktionsstörungen oder Programmierungsfehler bei 30 bis $40 \%$ der Patienten in der postoperativen Rehabilitation vor. Diese können im Rahmen der Rehabilitation festgestellt und ggf. auch beseitigt werden. Wichtig ist der Batteriezustand des Aggregats, um die Patienten rechtzeitig auf einen Aggregatwechsel einzustellen.

Ansonsten gelten während der Rehabilitation dieselben Richtlinien wie bei ICD- oder Herzschrittmacherpatienten ohne ein VAD. Insbesondere im Zusammenhang mit einer Elektrotherapie (z. B. Reizstromtherapie, TENS u. Ä.) ist auf die entsprechenden Kontraindikationen zu verweisen. Dasselbe gilt für alle anderen Arten von elektromagnetischen Interferenzen [77, 78].

In Tab. 5 sind Empfehlungen für den Umgang mit zusätzlich implantierten Geräten und Herzrhythmusstörungen bei VAD-Patienten während der Anschlussrehabilitation zusammengefasst.

\subsection{Besonderheiten der Medikation während der Rehabilitation von VAD-Patienten}

Grundsätzlich sollte zunächst überdacht werden, ob neben der VAD-abhängigen Medikation auch eine leitliniengerechte Therapie der jeweiligen Grunderkrankung des nativen Herzens (insbesondere der Herzinsuffizienz) erfolgversprechend ist. Dies gilt vor allem für Patienten, bei denen eine Erholung des erkrankten Myokards möglich erscheint (z. B. ,brigde to recovery“).

Eine effektive medikamentöse Therapie bildet die Basis jeder langfristig erfolgreichen Behandlung von VADPatienten und beginnt bereits vor der Implantation. Ein nachfolgendes regelmäßiges, komplexes Medikamentenregime, welches sowohl den Bedürfnissen des VAD (Antikoagulation, Volumenmanagement) als auch der kardialen Grunderkrankung und den Komorbiditäten Rechnung trägt, ist für eine erfolgreiche Langzeittherapie unerlässlich.

Die Anschlussheilbehandlung ist gut geeignet, die medikamentöse Einstellung für die Langzeittherapie zu optimieren.

Antikoagulation (MUST) Die Festlegung des therapeutischen Bereiches der INR-Einstellung zur Vermeidung thrombembolischer Komplikationen erfolgt unter Abwägung möglicher Blutungsrisiken. Das Antikoagulationsmanagement muss dabei die volle Komplexität der Anamnese berücksichtigen und wird in der Regel vom implantierten Zentrum vorgegeben. Die wesentlichen Einflussfaktoren für die INR-Einstellung sind die individuellen Begleiterkrankungen des Patienten und die Eigenschaften des Implantats. Als Standardmedikation werden zurzeit Phenprocoumon und Warfarin eingesetzt. Medikamentöse Alternativen sind in klinischen Studien bisher nicht ausreichend untersucht und können aus diesem Grund nicht empfohlen werden $[13,28]$.

Die Häufigkeit der INR-Messung, insbesondere im Rahmen der Langzeittherapie, wird unterschiedlich gehandhabt: Abhängig vom implantierenden Zentrum liegt die empfohlene Frequenz der INR-Messung bei einmal täglich bis einmal wöchentlich. Um die individuelle Führung der INR-Einstellung zu erleichtern, ist eine Selbstmessung des Patienten (nach entsprechender Schulung) anzustreben [13, 28, 79].

Generell muss immer eine Balance zwischen Thromboserisiko und Blutungsrisiko unter Berücksichtigung der klinischen Situation des Patienten und des Pumpentyps erreicht werden. Bei allen Patienten mit axialer Pumpe wurde ein erworbenes von-Willebrand-Syndrom nachgewiesen [80]. Dies kann zu einer erhöhten Blutungsneigung beitragen.

In der Regel werden vom implantierenden Zentrum sowohl der Wirkstoff als auch der therapeutische Bereich vorgegeben. Die Empfehlungen variieren in engen Grenzen. Die Antikoagulation wird in der Regel durch eine Thrombozytenaggregationshemmung ergänzt.

Der therapeutische Bereich der INR-Einstellung wird für das HeartMate II ${ }^{\circledR}$-System von Thorate ${ }^{\circledR}$ mit einem INR von 2,0-2,5 angegeben. Für das HVAD von HeartWare ${ }^{\circledR}$ wird 
Tab. 5 Umgang mit zusätzlich implantierten Geräten und Herzrhythmusstörungen

Maßnahme Rehabilitation bei LVAD-Patienten MoSCoW Bemerkung

Postoperative Kontrolle bei implantiertem ICD/CRT MUST

Kontrolle ICD/CRT durch die Rehabilitationsklinik

SHOULD

Parameter bzw. Maßnahmen, die bei einer Kontrolle von Herzschrittmachern, ICD oder CRT Aggregaten erhoben werden sollten:

Batteriestatus/Betriebsdauer

MUST

Status Implantationsstelle

MUST

Reizschwellen

MUST

Wahrnehmungsschwellen

MUST

Impedanzen

MUST

Aktuelle Programmierung

MUST

Gerätespeicher mit Analyse gespeicherter MUST

Rhythmusereignisse

Umprogrammierung der Geräte

MUST

ICD-Funktion an?

CRT-Funktion an?

Elektive Eingriffe bei Arrhythmien

Notfallmäßige Eingriffe bei Arrhythmien:

Externe Kardioverison

Interne Kardioversion bzw. antitachykardes Pacing

bei implantierten ICD

Medikamentöse Konversionen

Rhythmologische Kontrolle während der Re-

habilitation mittels EKG/LZ-EKG, ggf. notfall-

mäßige Elektrolytkontrolle

Möglichkeit eines Rhythmusmonitorings zur Überwachung (optimalerweise Monitorkontrolle von EKG, Sauerstoffsättigung, ggf. Blutdruckmessung) Invasives Überwachungsmonitoring bei Herzrhythmusstörungen (z. B. arterielle direkte Blutdruckmessung)

Kontaktaufnahme mit einem rhythmologischen

Zentrum mit EPU- und Ablationsmöglichkeit bei rezidivierenden rhythmogenen Ereignissen

Thematisierung psychologischer Folgen wiederholter ICD-Schocks bei der psychologischen Betreuung
Die Möglichkeit der Abfrage und Programmierung der wichtigsten Aggregatefirmen sollte in der Rehabilitation von Patienten mit VAD gegeben sein (Alternative: Externe Gerätekontrolle in enger Zusammenarbeit mit ambulanten Zentren oder dem zuweisenden Zentrum)
SHOULD

COULD Elektive Eingriffe sollten eher an das VAD-Zentrum verwiesen werden

MUST

SHOULD

MUST

MUST

MUST

COULD

Sollte eine invasive Kontrolle von Parametern notwendig werden, sollte der Patient notfallmäßig dem VAD-Zentrum zugewiesen werden.

SHOULD

SHOULD

Bei VAD-Patienten sollte wie bei anderen Patienten auch an die Möglichkeit psychischer Komorbiditäten durch ICD-Schocks gedacht werden (Angst, Depression, posttraumatische Belastungsreaktionen) in der Regel ein INR-Bereich von 2,0-3,0 empfohlen [13, 80-83]. Sollte der INR-Wert unterhalb des therapeutischen Bereichs nachgewiesen werden, wird nach Vorgabe des implantierenden Zentrums eine Überbrückung mit Standardoder niedermolekularem Heparin vorgenommen.

Bei nachgewiesener heparininduzierter Thrombopenie (HIT) wird auf Fondaparinux als subkutane Gabe bzw. Argatroban intravenös zurückgegriffen [13, 28, 79].

Thrombozytenaggregationshemmung (SHOULD) Die ergänzend eingesetzten Thrombozytenaggregationshemmer (ASS $100 \mathrm{mg}$, Clopidogrel $75 \mathrm{mg}$ ) werden nach zentrumsspezifischer Vorgabe mit einer Einnahmefrequenz von $3 \times$ bis $7 \times$ pro Woche dosiert. Neue Substanzgruppen (Ticagrelor oder Prasugrel) wurden bisher bei VADPatienten noch nicht getestet. Behandlungsziel ist eine $70 \%$ ige Inhibierung der Thrombozytenaggregation. Zur Kontrolle können Thrombozytenfunktionstests eingesetzt werden [84]. Komorbiditäten, die eine spezifische leitliniengerechte Thrombozytenaggregationshemmung verlangen, wie z. B. das DE-Stenting, ein vorausgegangener Apoplex 
oder eine pAVK, müssen bei der Präparateauswahl und der Dosierung berücksichtigt werden.

Behandlung einer systemischen Hypertension (MUST) Ein systemischer Hypertonus, der bereits vor der Entwicklung einer signifikanten Herzinsuffizienz bestanden hat, tritt häufig nach VAD-Implantation wieder auf. Hypertonus bedeutet eine Erhöhung der Nachlast und vermindert dadurch die Effizienz und Lebensdauer des VAD. Es kommt $\mathrm{zu}$ einer Abnahme des Pumpenflusses und damit zu einem geringeren Herzzeitvolumen. Der myokardiale Sauerstoffverbrauch steigt an, und der Grad einer Aortenklappeninsuffizienz kann zunehmen.

Evidenzbasierte Empfehlungen zur Behandlung eines Hypertonus bei VAD-Patienten bestehen nicht. Ein arterieller Mitteldruck von $60-80 \mathrm{mmHg}$ wird angestrebt $[13,28]$. ACE-Hemmer und ATII-Blocker reduzieren die Nachlast und sind daher als "first line“-Medikation sinnvoll. Grenzen der Einsetzbarkeit stellen dabei eine Niereninsuffizienz und eine Hyperkaliämie dar, insbesondere in der frühen postoperativen Phase. Betablocker, Kalziumkanalblocker und Diuretika können, falls notwendig, als weitere, ergänzende Antihypertensiva genutzt werden. Betablocker müssen jedoch mit Vorsicht bei einer rechtsventrikulären Dysfunktion und bei unzureichender Rekompensation der Herzinsuffizienz titriert werden.

Vorteile für den Einsatz von Aldosteronantagonisten bestehen vor allem im Hinblick auf die Vermeidung einer möglicherweise notwendig werdenden Kaliumsubstitution. Nitrate und direkte Vasodilatatoren wie Hydralazin können hilfreich sein bei der Reduzierung der Vor- und Nachlast des linken Ventrikels.

Behandlung von Arrhythmien (COULD) Patienten nach VAD-Implantation haben weiterhin strukturelle kardiale Veränderungen, die die Arrhythmiegefährdung erhöhen. Zusätzlich können Ansaugphänomene Herzrhythmusstörungen auslösen. Als antiarrhythmische Substanzen werden bevorzugt Betablocker und Amiodaron eingesetzt.

Vorhofflimmern führt in der Regel nur zu geringen klinischen Auswirkungen. Selbst ventrikuläre Tachykardien verändern die Hämodynamik nur im geringen Maße, solange die rechtsventrikuläre Funktion erhalten bleibt.

Sogar bei Kammerflimmern verliert der Patient nicht zwingend das Bewusstsein und kann mehr durch einen Defibrillatorschock beeinträchtigt sein als durch die ursächliche Arrhythmie. Allerdings sind sowohl Art und Ausmaß der Herzrhythmusstörungen als auch die Häufigkeit von Schockabgaben mit einer erhöhten Mortalität assoziiert.

Volumenmanagement und Rechtsherzinsuffizienz (SHOULD) Eine Rechtsherzschwäche hat direkte negative Auswirkung auf die Mortalität und die Effizienz der VAD-
Therapie. Ein optimierter Volumenstatus unter Einsatz von Diuretika ist bedeutsam für die Einstellung der Vorlast. Eine angepasste Flüssigkeitsbilanzierung hilft auch, Ansaugproblemen im linken Ventrikel vorzubeugen.

Weiteren Einfluss auf die rechtsventrikuläre Funktion übt der Lungenarteriendruck aus. Bei pulmonalem Hochdruck stehen unterschiedliche Wirkstoffgruppen zur pharmakologischen Korrektur zur Verfügung. Zu beachten hierbei sind die besonderen Verschreibungsvorschriften. Über die Dauer der Behandlung existieren bisher keine Studienergebnisse [85-87].

\subsection{Hygiene, Pflege und Monitoring bei VAD-Patienten in der Rehabilitation}

Die ersten drei Monate nach Implantation eines VAD stellen die Zeit mit der höchsten Gefährdung für eine ausgeprägte Infektion dar und bleiben die Achillesferse der mit einer Driveline ausgestatteten implantierbaren VADs [88]. Die bei einem VAD auftretenden Infektionen werden in

- VAD-spezifische,

- VAD-bezogene und

- nicht mit dem VAD in Zusammenhang stehende

Infektionen eingeteilt [89]. VAD-spezifische Infektionen schließen Infektionen ein, die spezifisch bei Patienten mit einem VAD sind und bei Patienten ohne ein VAD nicht auftreten. VAD-bezogene Infektionen schließen Infektionen ein, die auch bei Nicht-VAD-Patienten auftreten können, wobei die letztendliche Diagnose im Zusammenhang mit dem VAD steht. Nicht ursächlich mit einem VAD zusammenhängende Infektionen sind nicht an das Vorhandensein eines VAD-Systems gebunden und werden in diesem Artikel nicht behandelt [89, 90].

Das Vorliegen einer schwer beherrschbaren Allgemeininfektion oder einer ausgeprägten Infektion der Driveline, insbesondere mit multiresistenten Erregern, lässt eine Aufnahme in die Rehabilitation nicht zu.

Bei einer Besiedlung mit multiresistenten Erregern (z. B. VRE, MRSA, MRGN) muss die Aufnahmemöglichkeit in die Rehabilitationsklinik vorher besprochen werden und kann nur ggf. angepasst an das Hygienesetting der Rehabilitationsklinik erfolgen.

Patienten mit VAD-spezifischen oder VAD-bezogenen Infektionen können sich in einer Vielzahl von verschiedenen klinischen Bildern präsentieren, die die endgültige Diagnosestellung erschweren. Der erste Schritt sollte eine sorgfältige Anamnese und Beurteilung der Symptome sein. Dies soll zu einer frühen Erkennung und einem effizienten Diagnosealgorithmus führen, der die üblichen klinischen Untersuchungen bei Infektionsverdacht beinhaltet. 
Tab. 6 Einteilung und Maßnahmen bei Driveline-Infektionen während einer kardiologischen Anschlussheilbehandlung (Adaptiert nach Pistono [11])

\begin{tabular}{|c|c|c|c|c|c|c|}
\hline & $\begin{array}{l}\text { Lokale } \\
\text { Zeichen der } \\
\text { Infektion }\end{array}$ & Wundabstrich & Flüssigkeitsaustritt & $\begin{array}{l}\text { Fieber und/oder } \\
\text { Entzündungszeichen } \\
\text { im Blut }\end{array}$ & Blutkulturen & Behandlung \\
\hline Grad 0 & Nein & Negativ & Nein & Nein & Negativ & $\begin{array}{l}\text { Kochsalzspülung, nicht } \\
\text { alkoholisches Hautantiseptikum }\end{array}$ \\
\hline Grad 1 & $\mathrm{Ja}$ & Negativ & Nein & Nein & Negativ & Wundverband mit Silberfolie \\
\hline Grad 2 & $\mathrm{Ja}$ & Positiv & $\mathrm{Ja}$ & Nein & Negativ & $\begin{array}{l}\text { Orale oder intravenöse Anti- } \\
\text { biose, ggf. VAC-Verband, } \\
\text { Schmerzmittel bei Bedarf, } \\
\text { Überweisung ins implantierende } \\
\text { Zentrum erwägen }\end{array}$ \\
\hline Grad 3 & $\mathrm{Ja}$ & Positiv & $\mathrm{Ja}$ & $\mathrm{Ja}$ & Positiv & $\begin{array}{l}\text { Intravenöse Antibiose, ggf. } \\
\text { chirurgische Intervention, VAC- } \\
\text { Verband, ggf. Schmerzmittel, } \\
\text { Überweisung ins implantierende } \\
\text { Zentrum durchführen }\end{array}$ \\
\hline
\end{tabular}

Driveline-Infektionen Eines der langfristigen Risiken, die mit der VAD-Implantation verbunden sind, ist eine Infektion der Driveline oder der Pumpe selbst. Infektionen können zu einer lebensbedrohlichen Mediastinitis führen. Driveline-Infektionen können prinzipiell ausheilen, eine Infektion der Pumpe führt hingegen in der Regel zu einer Explantation des Systems. Die Angaben über die Häufigkeit einer Driveline-Infektion variieren in älteren Publikationen von $38 \%$ pro Patientenjahr bis $\mathrm{zu} 12 \%$ in neueren Veröffentlichungen [11, 91, 92].

Lokale Zeichen einer Infektion im Bereich der DrivelineAustrittsstelle sind Ausbildung eines Erythems, Überwärmung, Verhärtung, Aufweichung, Gewebeeinwuchs und Schmerz. Eine Driveline-Infektion lässt sich in drei Graduierungen einteilen. Bei einer Ausprägung Grad 2 und 3 ist auf jeden Fall die Kontaktaufnahme mit dem implantierenden Zentrum $\mathrm{zu}$ empfehlen, bei Grad 1 je nach Ausprägung des Befundes und Erfahrung der Rehabilitationsklinik. Weiterhin sollte bei dem Verdacht auf eine Geräteinfektion, Kanüleninfektion, Tascheninfektion oder tiefen Driveline-Infektion Kontakt mit dem implantierenden Zentrum aufgenommen werden. In Tab. 6 sind Einteilungen und empfohlene therapeutische Maßnahmen bei Driveline-Infektionen während einer Anschlussheilbehandlung von VAD-Patienten dargestellt.

Hinsichtlich des Keimspektrums einer VAD-spezifischen oder VAD-bezogenen Infektion gibt es nur wenig Literatur. In einer Übersicht von 2003 [91] finden sich hauptsächlich Staphylokokken und andere Biofilm produzierende Organismen, z. B. Pseudomonaden, Enterokokken und Candida, in einer Studie von Nienaber [93] hauptsächlich grampositive Kokken.

Da VAD-Patienten häufig längere Zeit vor dem Rehabilitationsaufenthalt in einer Intensivstation verbracht haben, ist von einer erhöhten Rate von Kolonisationen mit multiresistenten Erregern auszugehen. VAD-Patienten sind diesbezüglich als Risikopatienten zu betrachten [94].
Ein entsprechendes Screening insbesondere auf MRSA, gegebenenfalls auch auf MRGN, ist daher bei Aufnahme in die Rehabilitation empfehlenswert. Die Weitergabe von entsprechenden Untersuchungsbefunden und eine enge Zusammenarbeit mit den Hygienefachkräften sind notwendig. Auf strikte Basishygiene des betreuenden Personals ist $\mathrm{zu}$ achten.

Driveline-Pflege Die Durchtrittsstelle der Driveline ist wie eine offene Wunde zu behandeln, hier können Krankheitserreger eindringen und Wundinfektionen hervorrufen. Eine Wundpflege unter Beachtung der Standardhygienemaßnahmen hilft, dieses zu vermeiden. Dies bedeutet, dass der Verbandswechsel unter sterilen Bedingungen erfolgen muss.

In Tab. 7 sind Maßnahmen zur Vermeidung einer DrivelineInfektion bei VAD-Patienten zusammengefasst.

Der Patient muss in der richtigen Selbstpflege, einschließlich der Technik des Duschens und des Verbandswechsels, geschult werden. Dabei sollte die Verbandsart und Verbandstechnik aus dem implantierenden Zentrum weitergeführt werden und ggf. nur nach Rücksprache mit dem dortigen VAD-Koordinator verändert werden [13, 95]. Insgesamt stellen sich die Verbandsanleitungen als zentrumsspezifisch dar, sodass hier keine allgemein gültige Anleitung gegeben werden kann [13]. Insbesondere Infektionen der Driveline machen eventuell eine Änderung des Vorgehens der Verbandswechsel notwendig.

Wundabstriche sollen nur bei Bedarf (Infektionsverdacht oder Wundveränderungen) erfolgen. Bei Diagnose einer Wundheilungsstörung muss eine unmittelbare Kontaktaufnahme mit der implantierenden Klinik zwecks Absprache des weiteren Vorgehens erfolgen.

Übergang in den ambulanten Bereich Wenn der Patient selbst nicht in der Lage ist, die Wundversorgung durchzuführen, erfolgt dies durch Angehörige, den Hausarzt oder 
Tab. 7 Maßnahmen zur Vermeidung einer Driveline-Infektion von VAD-Patienten während einer kardiologischen Anschlussheilbehandlung: Überwachung und Behandlung (Adaptiert nach Pistono [11])

1. Fotografieren der Driveline-Austrittsstelle sollte bei der ersten Kontrolluntersuchung und bei jeder folgenden Kontrolluntersuchung durchgeführt werden, wenn folgende Aspekte vorliegen:

a. Anzeichen einer Infektion

b. Gewebeeinwuchs/Hypergranulation

c. Wundsekretion

d. Veränderung des umgebenden Gewebes

2. Spülung der Austrittsstelle mit 0,9\% NaCl-Lösung und Reinigung mit einer nichtalkoholischen Desinfektionslösung, z. B. Octenisept ${ }^{\mathbb{}}$ Lösung. Säuberung des Hautbereichs mit Kompressen, dabei von innen nach außen arbeiten. Ggf. zuvor Entnahme eines Wundabstrichs. Die Inspektion der Austrittsstelle erfolgt auf Zeichen einer Infektion, Gewebeabbau und Flüssigkeitsaustritt

3. Der Verband über der Driveline-Austrittsstelle muss sauber und trocken gehalten werden

4. Der Verbandswechsel muss unter sterilen Bedingungen unter Verwendung von Mundschutz, Schutzkittel und Einmalhandschuhen durchgeführt werden

Wenn die Wunde trocken und infektfrei ist:

a. Wundpflege und Verbandswechsel zu Beginn einmal täglich, bei unauffälligen Wundverhältnissen wöchentlich

Wenn die Wunde infiziert ist:

b. Wundpflege und Verbandswechsel täglich

5. Fixierung der Driveline: Zugfreie Positionierung und Fixierung der Bauchhalterung, um Läsionen oder Lösen zu verhindern. Polsterung und Stabilisierung des Kabels mit einer ausreichenden Anzahl von Kompressen unter- und oberhalb der Driveline

durch mobile Hilfsdienste nach Einweisung und Anleitung der Rehabilitationsklinik oder VAD-Ambulanz.

\subsection{Anwendung der ICF bei VAD-Patienten}

Die International Classification of Functioning, Disability and Health (ICF) betrachtet die funktionale Gesundheit eines Individuums vor dem Hintergrund seiner Körperfunktionen, Körperstrukturen, Fähigkeiten zu Aktivitäten und Teilhabe sowie seiner Kontextfaktoren (Barrieren oder Förderfaktoren). Beeinträchtigungen können erfasst und bei Bedarf auch quantifiziert werden. Eine umfassende Darstellung von theoretischem Hintergrund, Entwicklung, Zielen, Nutzen und praktischem Einsatz findet sich in der Originalpublikation der WHO [96]. Im klinischen Alltag wird die ICF vor allem als theoretisches Konstrukt zur Betrachtung von Gesundheitsproblemen und deren Auswirkungen sowie zur Berufsgruppen-übergreifenden Kommunikation verwendet [97]. Auch zur Abstimmung der Behandlungsziele zwischen Behandlungsteam und Patient kann die ICF im Sinne einer gemeinsamen Sprache hilfreich sein.

Seit der Einführung der ICF im Jahr 2001 wirkt die WHO darauf hin, die Prinzipien der ICF in allen Bereichen des Gesundheitswesens zunehmend bekannt zu machen und umzusetzen. Dieser Vorgang ist im rehabilitativen Sektor bereits fortgeschritten, nicht zuletzt deswegen, weil in diesem Bereich Interdisziplinarität, partizipative Entscheidungsfindung und Berücksichtigung von Kontextfaktoren eine große Rolle spielen. Um dies zu betonen und voranzutreiben, werden die Leistungserbringer der Rehabilitation auch durch ihre Kostenträger zur Anwendung der ICF verpflichtet. In der Akutmedizin ist die ICF hingegen weit weniger eingeführt, teilweise sogar unbekannt.
VAD-Patienten weisen regelhaft gravierende Einschränkungen in mehreren Lebensbereichen auf, die in den Komponenten der ICF, ihren Domänen und Kategorien abgebildet werden. Die Anwendung der ICF kann durch den Einsatz eines Core-Sets erleichtert werden [98, 99], das eine Auswahl der für VAD-Patienten relevanten Kategorien enthält und auf Kategorien verzichtet, die für den Regelfall unbedeutend sind. Um die Gesundheitsprobleme des VADPatienten in ihrer Gesamtheit zu erfassen, müssen zumindest die in Tab. 8 genannten Kategorien durch die Mitglieder der therapeutischen Teams während der Rehabilitation überprüft werden (MUST). Die Items der Tab. 8 können somit als Core-Set für die Erfassung von VAD-Patienten dienen, das alle MUST-Kriterien enthält. Zu ihrer Überprüfung sind eigen- und fremdanamnestische Angaben, klinische, paraklinische und apparative Befunde sowie im Bedarfsfall auch Ergebnisse geeigneter Assessmentverfahren heranzuziehen. Abhängig vom Einzelfall und von klinikspezifischen Standards können selbstverständlich alle weiteren Kategorien und die vollständige Differenzierung der gesamten ICF zur Erfassung der individuellen Gesundheitsprobleme und zur Entwicklung erweiterter klinikeigener Core-Sets eingesetzt werden.

Die Beurteilung der funktionalen Gesundheit aus Sicht der ICF erlaubt es auch, Einschränkungen bzw. Normabweichungen von Körperfunktionen und -strukturen, Aktivitäten, Teilhabe und Kontextfaktoren in Bezug auf die zugeordnete Kategorie zu quantifizieren und das Ergebnis als Zifferncode zu dokumentieren. Dieser Vorgang wird als ICF-Codierung bezeichnet. Bei Verwendung des in Tab. 8 dargestellten Core-Sets wäre jeder Patient durch 59 mindestens fünf- bis siebenstellige Codes zu charakterisieren. Durch mehrere Codierungen zu verschiedenen Zeitpunkten können Krankheitsverlauf bzw. 
Tab. 8 Mindeststandard der Kategorien der ICF-Kurzversion [100], die bei der Anschlussheilbehandlung von VAD-Patienten zu beachten sind (von der Autorengruppe in Konsensbildung entwickelte Minimalanforderung)

\begin{tabular}{|c|c|c|}
\hline & Items der ICF-Kurzversion & Beispiel mit Bezug zu VAD-Patienten \\
\hline \multicolumn{3}{|c|}{ Körperfunktionen } \\
\hline b110 & Funktionen des Bewusstseins & Quantitative und qualitative Bewusstseinslage \\
\hline b114 & Funktionen der Orientierung & Orientierung zu Person, Ort, Zeit, Situation \\
\hline b130 & Funktionen der psychischen Energie und des Antriebs & Motivation, Appetit \\
\hline b144 & Funktionen des Gedächtnisses & Kurz- und Langzeitgedächtnis \\
\hline b152 & Emotionale Funktionen & Angst, Depressivität \\
\hline b235 & Vestibuläre Funktionen & Schwindel \\
\hline b280 & Schmerz & Schmerzempfindung \\
\hline b410 & Herzfunktionen & Herzrhythmus, Pumpfunktion \\
\hline b420 & Blutdruckfunktionen & Hypo-/Hypertonie, Blutdruckstabilität \\
\hline b430 & Funktionen des hämatologischen Systems & Blutbildung, Blutgerinnung \\
\hline b440 & Atmungsfunktionen & Ventilation, Gasaustausch \\
\hline b455 & Funktionen der kardiorespiratorischen Belastbarkeit & Ausdauerleistung \\
\hline b5 510 & Funktionen der Nahrungsaufnahme & Essen, Trinken \\
\hline b525 & Defäkationsfunktionen & Stuhlfrequenz und Konsistenz \\
\hline b550 & Funktionen der Aufrechterhaltung des Körpergewichts & Kachexie, Adipositas \\
\hline b545 & Funktionen des Wasser-, Mineral- und Elektrolythaushaltes & Hydratation, Elektrolytstatus \\
\hline b610 & Harnbildungsfunktionen & Nierenfunktion \\
\hline b620 & Miktionsfunktionen & Miktionsfrequenz, -beschwerden \\
\hline b710 & Funktionen der Gelenkbeweglichkeit & Umfang der Gelenkbeweglichkeit \\
\hline b730 & Funktionen der Muskelkraft & Kraft von Muskeln und Muskelgruppen \\
\hline b740 & Funktionen der Muskelausdauer & Ausdauer von Muskeln und Muskelgruppen \\
\hline $\mathrm{b} 820$ & Heilfunktion der Haut & Heilung von Driveline-Austrittsstelle, OP-Narben \\
\hline \multicolumn{3}{|c|}{ Körperstrukturen } \\
\hline s410 & Struktur des kardiovaskulären Systems & Strukturschäden von Herz, Arterien, Venen \\
\hline s430 & Struktur des Atmungssystems & Strukturschäden des Atmungssystems \\
\hline s760 & Struktur des Rumpfes & OP-Narben am Rumpf \\
\hline s810 & Struktur der Hautregionen & Driveline-Durchtritt, OP-Narben \\
\hline \multicolumn{3}{|c|}{ Aktivitäten und Partizipation (Teilhabe) } \\
\hline $\mathrm{d} 230$ & Die tägliche Routine durchführen & Alltagsaufgaben planen und bewältigen \\
\hline $\mathrm{d} 240$ & Mit Stress und andern psychischen Anforderungen umgehen & Stress, Störungen und Krisen bewältigen \\
\hline $\mathrm{d} 410$ & Eine elementare Körperposition wechseln & Sich aufsetzen, aufstehen \\
\hline $\mathrm{d} 440$ & Feinmotorischer Handgebrauch & Handhabung des VAD-Kontrollers \\
\hline $\mathrm{d} 445$ & Hand- und Armgebrauch & Umgang mit dem VAD \\
\hline $\mathrm{d} 450$ & Gehen & Kurze oder lange Strecken gehen \\
\hline $\mathrm{d} 455$ & Sich auf andere Weise fortbewegen & Treppen steigen \\
\hline d460 & Sich in verschiedenen Umgebungen fortbewegen & Spazieren gehen drinnen und draußen \\
\hline $\mathrm{d} 465$ & Sich unter Verwendung von Geräten/Ausrüstung fortbewegen & Rollatorgebrauch \\
\hline $\mathrm{d} 510$ & Sich waschen & Sich waschen, duschen, abtrocknen \\
\hline $\mathrm{d} 520$ & Seine Körperteile pflegen & Haut, Haare und andere Körperregionen pflegen \\
\hline d530 & Die Toilette benutzen & $\begin{array}{l}\text { Planung und Durchführung des Toilettengangs, an- } \\
\text { schließende Körperreinigung }\end{array}$ \\
\hline d5 540 & Sich kleiden & Sich an- und ausziehen \\
\hline d550 & Essen & Essen \\
\hline d560 & Trinken & Trinken \\
\hline d570 & Auf seine Gesundheit achten & $\begin{array}{l}\text { Lebensstilmaßnahmen umsetzen, Kontrollunter- } \\
\text { suchungen wahrnehmen }\end{array}$ \\
\hline d620 & Waren und Dienstleistungen des täglichen Bedarfs beschaffen & Einkaufen gehen \\
\hline d630 & Mahlzeiten vorbereiten & Kochen \\
\hline d640 & Hausarbeiten erledigen & $\begin{array}{l}\text { Hauarbeiten erledigen wie Wäsche waschen, Wohn- } \\
\text { raum reinigen, Müll entsorgen }\end{array}$ \\
\hline d760 & Familienbeziehungen & $\begin{array}{l}\text { Beziehungen zu Verwandten aufbauen und } \\
\text { aufrechterhalten }\end{array}$ \\
\hline $\mathrm{d} 850$ & Bezahlte Tätigkeit & Arbeiten gehen \\
\hline
\end{tabular}


Tab. 8 (Fortsetzung)

\begin{tabular}{|c|c|c|}
\hline & Items der ICF-Kurzversion & Beispiel mit Bezug zu VAD-Patienten \\
\hline $\mathrm{d} 870$ & Wirtschaftliche Eigenständigkeit & $\begin{array}{l}\text { Private oder öffentliche Quellen zum Erhalt } \\
\text { wirtschaftlicher Eigenständigkeit nutzen }\end{array}$ \\
\hline d910 & Gemeinschaftsleben & Feierlichkeiten besuchen, soziale Aktivitäten \\
\hline d920 & Erholung und Freizeit & An Hobby, Unterhaltung, Tourismus teilhaben \\
\hline \multicolumn{3}{|c|}{ Umweltfaktoren (Kontextfaktoren) } \\
\hline e110 & Produkte und Substanzen für den persönlichen Verbrauch & Medikamentöse Versorgung \\
\hline e115 & $\begin{array}{l}\text { Produkte und Technologien zum persönlichen Gebrauch im } \\
\text { täglichen Leben }\end{array}$ & $\begin{array}{l}\text { Implantate (Assist device), Hilfsmittel, } \\
\text { Stromversorgung }\end{array}$ \\
\hline e120 & $\begin{array}{l}\text { Produkte und Technologien zur persönlichen Mobilität drinnen } \\
\text { und draußen und zum Transport }\end{array}$ & Rollator-Versorgung \\
\hline e310 & Engster Familienkreis & $\begin{array}{l}\text { Physische und emotionale Unterstützung im engsten } \\
\text { Familienkreis }\end{array}$ \\
\hline e340 & Persönliche Hilfs- und Pflegepersonen & Haushaltshilfe \\
\hline e355 & Fachleute der Gesundheitsberufe & Ärztliche und pflegerische Versorgung \\
\hline e410 & $\begin{array}{l}\text { Individuelle Einstellungen der Mitglieder des engsten } \\
\text { Familienkreises }\end{array}$ & Einstellung der Familie zur Assist-device-Therapie \\
\hline e570 & $\begin{array}{l}\text { Dienste, Systeme und Handlungsgrundsätze der sozialen } \\
\text { Sicherheit }\end{array}$ & $\begin{array}{l}\text { Kompensation für Erwerbsminderung, } \\
\text { Schwerbehinderung }\end{array}$ \\
\hline e580 & $\begin{array}{l}\text { Dienste, Systeme und Handlungsgrundsätze des } \\
\text { Gesundheitswesens }\end{array}$ & $\begin{array}{l}\text { Ressourcen und Allokation akuter, chronischer und } \\
\text { rehabilitativer Versorgung }\end{array}$ \\
\hline
\end{tabular}

Fortschritte im Rehabilitationsprozess abgebildet und veranschaulicht werden.

Aufgrund des hohen damit verbundenen Aufwands gehört die ICF-Codierung nicht zum Standardumfang der Anschlussheilbehandlung von VAD-Patienten. Zurzeit bleibt sie wissenschaftlichen Fragestellungen und Modellprojekten vorbehalten.

\subsection{Bewegungstherapie im Rahmen der Rehabilitation nach VAD-Implantation}

\section{Begriffsbestimmung}

Die Bewegungstherapie wird definiert als eine „,medizinisch indizierte und ärztlich verordnete Bewegung, die vom Fachtherapeuten geplant und dosiert, gemeinsam mit dem Arzt kontrolliert und mit dem Patienten alleine oder in der Gruppe durchgeführt wird“" [101].

Die Physiotherapie umfasst die physiotherapeutischen Verfahren der Bewegungstherapie sowie die physikalische Therapie und wird in der Regel als Einzeltherapie durchgeführt.

Die Sporttherapie ist ,eine bewegungstherapeutische Maßnahme, die mit geeigneten Mitteln des Sports gestörte körperliche, psychische und soziale Funktionen kompensiert, regeneriert, Sekundärschäden vorbeugt und gesundheitlich orientiertes Verhalten fördert. Sie beruht auf biologischen Gesetzmäßigkeiten und bezieht besonders Elemente pädagogischer, psychologischer und sozialtherapeutischer Verfahren ein und versucht eine überdauernde Gesundheitskompetenz zu erzielen“ [101]. Sie wird in der Regel in Gruppen durchgeführt.
In der Praxis der Rehabilitation werden diese Begriffe unter dem Sammelbegriff Bewegungstherapie oft unscharf voneinander abgegrenzt. Es bestehen zudem Überschneidungen sowie fließende Übergänge zwischen diesen Therapiebereichen. Bei der rehabilitativen Behandlung des VAD-Patienten gilt dies sowohl für die inhaltliche Gestaltung als auch für die fachtherapeutische Betreuung. Diese Bereiche werden daher gemeinsam in einem Abschnitt abgehandelt.

Besonderheiten bei VAD-Patienten VAD-Patienten sind in der Regel ein heterogenes Patientenkollektiv. Das betrifft insbesondere die Altersverteilung, Ätiologie, Komorbiditäten, VAD-Modelle, Postimplantationszeit und den Dekonditionierungsgrad. Durch die VAD-Implantation wird die Sauerstoffversorgung deutlich verbessert, was in der Regel mit einer Verbesserung der maximalen Sauerstoffaufnahme [102] und der NYHA-Klassifikation [103] verbunden ist. Trotzdem bleibt die Leistungsfähigkeit jedoch nach der Implantation zunächst deutlich reduziert (11-20 ml/kg/min) [104]. Durch eine häufig lange Krankheitsgeschichte ist der Körper insgesamt in einem erheblich dekonditionierten Zustand. Dies betrifft insbesondere die Skelettmuskulatur, die bei einer langandauernden Herzinsuffizienz durch katabole Prozesse beeinflusst wird. Ein Vorgang, der häufig durch langdauernde Immobilisation und Bettlägerigkeit dieser Patientengruppe weiter verstärkt wird. Bei VAD-Patienten ist daher aufgrund einer muskulären Dekonditionierung sowie der auch postimplantativ geringeren Herzleistung mit frühzeitigem Auftreten von Dyspnoe, Müdigkeit und Erschöpfung zu rechnen [13, 105]. Hinzu kommt ggf. eine Mobilitätseinschränkung auf Grund 
des VAD-Controllers und der Driveline. Des Weiteren kann bei dieser Patientengruppe eine Beeinträchtigung der pulmonalen Kapazität vorliegen. Die besondere psychische Herausforderung, welche mit der Implantation verbunden ist, kann Tendenzen zu Ängstlichkeit und Depressivität verstärken. Die adäquate Betreuung von VAD-Patienten stellt somit eine Herausforderung für die Bewegungstherapie dar. Sie erfordert in besonderem Maße individuell angepasste und kontrollierte bewegungstherapeutische Maßnahmen, die häufig nur durch eine Einzelbetreuung zu gewährleisten sind.

Physikalische Therapie Ziele der physikalischen Therapie sind die Linderung von Schmerzen und Funktionseinschränkungen an Körperstamm und Extremitäten sowie in Bezug auf die Atemphysiotherapie die Verbesserung der respiratorischen Funktion und der bronchialen Sekretelimination. Die physikalische Therapie umfasst eine Reihe passiver Maßnahmen, die ohne Willkürinnervation des Patienten erfolgen. Auch bei passiven Maßnahmen kann es durch Änderung insbesondere von Gefäßwiderstand und venösem Rückstrom zu Einflüssen auf die Hämodynamik kommen.

Physio- und Bewegungstherapie Ziele der Physio- und Bewegungstherapie sind die Reduktion von Funktionseinschränkungen, die Verbesserung von Mobilität, Muskelkraft, Ausdauer und Koordination sowie die Rückbildung der Skelettmuskelatrophie. Übergeordnetes Ziel ist die Verbesserung der Lebensqualität. Dies wird angestrebt durch eine Verbesserung der körperlichen Leistungsfähigkeit, insbesondere der funktionalen Kapazität und der Alltagsbelastbarkeit. Wichtig ist, dass der Patient im Rahmen der Bewegungstherapie den Umgang mit dem VAD-System bei körperlicher Aktivität und bei Belastungen im Alltag, in der Freizeit und ggf. im Beruf lernt. Dabei geht es einerseits um Sicherheitsaspekte im Umgang mit dem VAD und anderseits um die Schulung von Belastungsempfinden und Belastungsbeurteilung des Patienten, wodurch eine realistische Selbsteinschätzung angestrebt wird. Hierdurch können die Krankheitsbewältigung und die seelische Stabilisierung unterstützt und ein Beitrag zum Abbau von Bewegungsangst geleistet werden. Die Bewegungstherapie leistet somit einen wesentlichen Beitrag zur Wiederherstellung und Verbesserung der Teilhabe.

In aktuellen Positionspapieren, Empfehlungen und Guidelines werden mögliche bewegungstherapeutische Maßnahmen mit dieser Patientengruppe zwar behandelt, aufgrund der geringen Evidenz werden jedoch nur vage Empfehlungen ausgesprochen [10, 12, 13, 50, 106, 107]. Bis jetzt haben nur wenige Arbeitsgruppen Erfahrungen mit VAD-Patienten gesammelt, und diese zudem meist mit sehr kleinen, heterogenen Patientengruppen [53, 108-113].
Diese Studien berichten über Erfahrungen mit aerobem Ausdauertraining in Form von Geh-, Fahrradergometerund Laufbandtraining, das sowohl nach der Dauer- als auch nach der Intervallmethode durchgeführt wurde. Hierdurch wurde die maximal erreichte Leistung (Watt ${ }_{\max }$ ) $[108,112$, 114], die $\mathrm{VO}_{\text {2peak }}[108,109,111,112,114]$, die 6-MinutenGehstrecke [108, 111], sowie die Lebensqualität [108, $109,111,114]$ verbessert. Alle Studien berichten über eine sichere Durchführung der Bewegungstherapie, wobei zu beachten ist, dass hier ein sehr stark selektiertes Patientenkollektiv eingeschlossen wurde [53, 104, 112, 115, 116]. In fünf Untersuchungen wurde das Ausdauertraining durch ein Krafttraining ergänzt, welches ebenfalls von den Patienten gut toleriert wurde [108, 109, 112, 113, 117, 118]. In einer Studie wurde das Ausdauer- und Krafttraining durch ein computerunterstütztes Inspirationstraining erweitert, das zu einer signifikanten Verbesserung der Atemeffizienz führte [111]. Zusammenfassend erlauben die Ergebnisse bisheriger Untersuchungen keine verlässlichen Aussagen über die Effektivität bewegungstherapeutischer Maßnahmen. Sie liefern jedoch den Hinweis, dass diese Patientengruppen zumindest kurzfristig von individuell angepasster Bewegungstherapie profitieren. Ergebnisse zu Langzeiteffekten von bewegungstherapeutischen Maßnahmen liegen bisher nicht vor. In diesen Untersuchungen wurden bewegungstherapeutische Maßnahmen in unterschiedlichen Phasen der Rehabilitation, insbesondere hinsichtlich der Zeitspanne nach der Implantation, durchgeführt. Die Frage, wie früh nach der Implantation der Patient belastet werden kann bzw. wann er mit einer angepassten Bewegungstherapie beginnen kann und sollte, lässt sich somit noch nicht beantworten. In einigen Zentren wurde bereits 2 Wochen nach Implantation mit angepassten Trainingsmaßnahmen begonnen. Keine Untersuchung hat sich jedoch mit dem Aspekt der Sicherheit explizit beschäftigt.

Aktuelle Empfehlungen zur Bewegungstherapie bei VAD-Patienten basieren somit fast ausschließlich auf den Erfahrungen, die mit bewegungstherapeutischen Interventionen bei Herzinsuffizienzpatienten im Laufe der letzten Jahre gesammelt wurden [12, 119].

\section{Planung und Durchführung der Physio-/Bewegungs-} therapie Für die Planung und Durchführung der Physio-/ Bewegungstherapie kann es hilfreich sein, VAD-Patienten basierend auf den Ergebnissen des 6-Minuten-Gehtests (6-MWD) zu Therapiegruppen zu klassifizieren und geeigneten therapeutischen Maßnahmen und/oder Therapiegruppen zuzuordnen. Eine mögliche Einteilung wäre: Einzeltherapie (auf dem Zimmer) 6-MWD $<100$ m; Kleingruppentherapie (3-6 Patienten) (ggf. mit Rollator) 6-MWD =100-300 m; Gruppentherapie (>6 Patienten) (ohne Gehhilfe) 6-MWD >300 m. Eine zusätzliche Möglichkeit wäre 
die Klassifikation nach dem Mobilitätsgrad (Zimmer-mobil; Flur-mobil, Treppen-mobil) [10].

Die Physio- und Bewegungstherapie sollte ausschließlich von erfahrenen und gut geschulten Therapeuten durchgeführt werden. Diese müssen mit den angewandten Systemen vertraut sein, um Alarme und Hinweise des Controllers sicher deuten zu können, sowie im speziellen Notfallmanagement geschult sein. Essenziell ist, dass die Therapeuten mit den allgemeinen Sicherheitsaspekten der Durchführung der Bewegungstherapie bei dieser Patientengruppe vertraut sind. Hierzu gehören: Kontrolle der Akkus (Ladezustand, Ersatzakkus vorhanden?); Berücksichtigung der DrivelineLänge und -Lage (besteht die Gefahr, dass die Driveline durch die Übungsauswahl und Durchführung inadäquaten Zug, Knick oder Torsion erfährt?); Wahl und Kontrolle der Befestigung des Controllers und der Akkumulatoren (hier sind Umhängetaschen ungünstig, da ein Ablegen der Taschen durch die kurze Driveline nur bedingt möglich ist). Vor Antritt der Rehabilitation sollten die Patienten nach Möglichkeit mit Gürteltasche oder Safarijacke ausgerüstet werden, da dies die Bewegungsfreiheit verbessert [50, 107].

Bei frisch sternotomierten Patienten muss die Stabilität des Sternums gesichert sein. Beim Abhusten sollte das Sternum durch Gegendruck mit einem weichen Gegenstand (z. B. Kissen) geschient werden. Weiterhin sollten bei dieser Patientengruppe vermieden werden: Heben, Drücken oder Ziehen von mehr als $5 \mathrm{~kg}$ mit der oberen Extremität, gleichzeitige bilaterale Elevation oder Abduktion beider Arme über $90^{\circ}$, starke Anspannung der abdominalen Muskelgruppen, die im Bereich der unteren Thoraxapertur inserieren, sowie exzessive Valsalva-Manöver. Bei unkompliziertem Heilungsverlauf können diese Einschränkungen nach zentrumsspezifischen Standards gelockert werden [120]. In der Regel wird die volle Belastbarkeit des Sternums nach 3 Monaten angenommen. Vorteilhaft ist die minimalinvasive Implantationstechnik mit erhaltener Kontinuität des Sternums, bei der diese Einschränkungen entfallen.

Relevante Kontraindikationen für bewegungstherapeutische Maßnahmen speziell für dieses Patientenkollektiv sind nicht publiziert. Es gelten die für Herzinsuffizienzpatienten publizierten Kontraindikationen [119]. Voraussetzung für die Aufnahme der Bewegungstherapie ist, dass der Patient hämodynamisch stabil ist. Ausschlusskriterien für die Aufnahme der Bewegungstherapie sind niedriger Volumenstatus mit Orthostasereaktion, Blutungen, Zeichen einer systemischen Infektion, ventrikuläre Arrhythmien und/oder VAD-Probleme.

Es gelten die üblichen Abbruchkriterien der Bewegungstherapie im Rahmen der kardiologischen Rehabilitation $[12,119]$. Für VAD-Träger sind bei der Bewegungstherapie zudem spezielle Abbruchkriterien zu beachten. Diese sind: eine Pumpenflussminderung $<3$ 1/Minute; ein Anstieg des Energiebedarfs der Pumpe in Watt (Thrombusgefahr); eine Sauerstoffsättigung $<90 \%$ (Pulsoxymeter); Blutungen (z. B. Nasenblutungen) [13, 50]. Bei der Durchführung der Bewegungstherapie sind grundsätzlich schnelle Lagewechsel zu vermeiden (z. B. vom Sitzen in den Stand mit rascher Blutvolumenverschiebung). Alle Aktivitäten, die zu einer unkontrollierten, inadäquaten Belastung des Systems führen könnten, sind ungeeignet (Kontaktsport, Spielformen etc.).

Als geeignete und sinnvolle bewegungstherapeutische Maßnahmen gelten aerobes Ausdauertraining nach der Dauermethode und/oder Intervallmethode (Ergo-

Tab. 9 Empfohlene aktive Maßnahmen der Physiotherapie/Bewegungstherapie in der frühen Phase der Rehabilitation in Abhängigkeit vom erreichten funktionellen Status (modifiziert nach [10, 121])

\begin{tabular}{|c|c|c|c|}
\hline Funktioneller Status & Merkmale & $\begin{array}{l}\text { Notwendige physiotherapeutische Maß- } \\
\text { nahmen (MUST) }\end{array}$ & $\begin{array}{l}\text { Optionale physiotherapeutische Maß- } \\
\text { nahmen (COULD) }\end{array}$ \\
\hline $\begin{array}{l}\text { Bettlägerig bis } \\
\text { Rollstuhl-fähig }\end{array}$ & $\begin{array}{l}\text { Unfähig ohne fremde Hilfe } \\
\text { aufzustehen }\end{array}$ & $\begin{array}{l}\text { Einzel-KG im Bett/am Bettrand, Trans- } \\
\text { ferübungen, Sitz-/Stehbalance-Übungen } \\
\text { Atemgymnastik }\end{array}$ & Atemvolumentraining \\
\hline Zimmer-mobil & $\begin{array}{l}\text { In der Lage, alleine aufzu- } \\
\text { stehen und im Zimmer z. B. } \\
\text { zur Sanitäreinheit zu gehen }\end{array}$ & $\begin{array}{l}\text { Einzel-KG mit den Elementen: } \\
\text { Atemgymnastik, Mobilisierung, } \\
\text { leichte kräftigende Übungen, leichte } \\
\text { Ausdauerbelastungen, einfache } \\
\text { Koordinationsübungen }\end{array}$ & $\begin{array}{l}\text { Pedalo-Training, Motomed }{ }^{\circledR} \text {-Training, } \\
\text { Bett-Ergometer passiv/aktiv }\end{array}$ \\
\hline Flur-mobil & $\begin{array}{l}\text { In der Lage, selbstständig } \\
\text { über den Flur zu gehen, } \\
\text { ggf. unter Verwendung von } \\
\text { Hilfsmitteln, wie z. B. eines } \\
\text { Rollators }\end{array}$ & $\begin{array}{l}\text { Hocker-KG mit Atemgymnastik, } \\
\text { kräftigende Übungen, Ausdauer- } \\
\text { belastungen, beweglich machende } \\
\text { Übungen, Koordinationsübungen }\end{array}$ & $\begin{array}{l}\text { Motomed }^{\circledR} \text {-Training, Training mit } \\
\text { elastischen Bändern, leichtes Hantel- } \\
\text { Training, Fahrradergometer-/Laufband- } \\
\text { Training, Gehtraining }\end{array}$ \\
\hline Treppen-mobil & $\begin{array}{l}\text { Ohne fremde Hilfe in der } \\
\text { Lage, eine Etage Treppen zu } \\
\text { steigen }\end{array}$ & $\begin{array}{l}\text { Bewegungstherapeutische Maßnahmen } \\
\text { (i.e. Ausdauer- und Kraftausdauertraining) } \\
\text { wie im Abschn. X beschrieben }\end{array}$ & $\begin{array}{l}\text { Motomed }^{\circledR} \text {-Training, Training mit } \\
\text { elastischen Bändern, leichtes Hantel- } \\
\text { Training, Kraftausdauertraining am } \\
\text { Gerät, Fahrradergometer-/Laufband- } \\
\text { Training, Gehtraining, Übung und } \\
\text { Training außerhalb der Klinik (z. B. } \\
\text { Terrain-Training) }\end{array}$ \\
\hline
\end{tabular}


metertraining mit Monitoring), Gehtraining (bis hin zu Wanderungen) ggf. auf dem Laufband, niedrig dosiertes bis moderates Krafttraining und gymnastische Übungen (einschließlich Koordination, Flexibilität und Übungen zur Schulung der Körperwahrnehmung). Bei Bedarf können zudem ein spezielles ATL-Training (siehe Ergotherapie, Abschn. 2.11) und/oder respiratorisches Training sinnvoll und hilfreich sein.

In der frühen Rehabilitation nach VAD-Implantation stehen physiotherapeutische Maßnahmen im Vordergrund. Diese sollten 5-7-mal pro Woche erfolgen. Bei voll mobilisierten Patienten ist eine Reduktion der Häufigkeit auf 2-3-mal pro Woche möglich.

Die Auswahl aktiver physiotherapeutischer Maßnahmen erfolgt in Abhängigkeit vom funktionellen Status des Patienten. Unter praktischen Gesichtspunkten sind vier Stadien globaler Funktionseinschränkungen zu unterscheiden, die durch ärztliche, pflegerische und physiotherapeutische Beurteilung festzustellen sind. Diese sind zusammen mit den daraus abzuleitenden notwendigen und optionalen physiotherapeutischen/bewegungstherapeutischen Maßnahmen in Tab. 9 aufgelistet.

Im Bedarfsfall können die aktiven physiotherapeutischen Maßnahmen durch passive Maßnahmen ergänzt werden. Als solche kommen beispielsweise Ausstreichmassagen bei Muskelverspannungen, schmerzhaftem Muskelhartspann oder Myogelosen, bei nichtkardialen Ödemen der Extremitäten auch Lymphdrainagen in Betracht. Dabei ist die Mobilisation großer Flüssigkeitsmengen wegen der Gefahr einer kardialen Dekompensation zu vermeiden.

Bei spezieller Indikation können Physiotherapeuten auf ärztliche Anordnung auch Wärme-, Kälte- oder Elektrotherapien durchführen. Dabei ist strikt darauf zu achten, dass keine Teile des VAD über Betriebstemperatur erwärmt, unter Betriebstemperatur abgekühlt oder schädlichen elektromagnetischen Feldstärken ausgesetzt werden. Dies ist in der Regel gewährleistet, wenn die Körperregion, in die das VAD implantiert ist (bei den heute üblichen Systemen der Thorax), von der Behandlung ausgeschlossen bleibt. Die Höhe der Feldstärke, die für ein VAD schädlich sein kann, variiert von Device zu Device. Moderne Axial- und Zentrifugalpumpen sind defibrillationsfest und gegenüber elektromagnetischen Belastungen wenig empfindlich. Verbindliche Angaben zu tolerierten Feldstärken des jeweiligen VAD können zurzeit nur die Hersteller geben.

Wegen möglicher unerwünschter Einflüsse auf die Hämodynamik sind großflächige Wärmeanwendungen zu vermeiden.

Aerobes Ausdauertraining Im Rahmen der kardiologischen Rehabilitation ist aerobes Ausdauertraining nach der Dauermethode Standard. Die Sicherheit und Effektivi- tät dieser Trainingsform gilt bei Patienten mit chronischer Herzinsuffizienz bereits als gesichert [119].

Unter Berücksichtigung der für den einzelnen VADPatienten besonderen Gegebenheiten können die hierfür publizierten Empfehlungen zur Orientierung herangezogen werden [119]. Im Regelfall wird dieses Training als individuell angepasstes Ergometertraining mit Monitoring durchgeführt. Dies ermöglicht eine gute Abstufbarkeit der Belastung und Überwachung von EKG und ggf. der Sauerstoffsättigung (Pulsoxymeter) und des Blutdruckes während der Belastung.

In Deutschland, Österreich und den Niederlanden existieren zudem in dieser Patientengruppe Erfahrungen mit aerobem Ausdauertraining nach der Intervalltrainingsmethode. Am häufigsten angewandt wird hier das 1-zu-2Protokoll, bei dem sich kurze Belastungsphasen (20-30 s) mit doppelt so langen Erholungsphasen (40-60 s mit geringer bzw. ohne Belastung) abwechseln. Die Anwender berichten über eine gute Akzeptanz, Toleranz und Umsetzbarkeit dieser Trainingsform. Für diese Methodik gilt, wie für das Dauertraining, dass sie nicht ausreichend wissenschaftlich in ihren Auswirkungen bei dieser Patientengruppe überprüft wurde [112]. In Ergänzung zum Ergometertraining sollten andere Ausdauerbelastungsformen, insbesondere Gehtraining (Gehen auf dem Gang, auf dem Klinikgelände) mit oder ohne Gehhilfe integriert werden. Die Empfehlungen zum Gehtraining müssen differenziert nach den individuellen Voraussetzungen des Patienten erfolgen. Dies betrifft insbesondere die angestrebte Gehstrecke, die Gehgeschwindigkeit, die Belastungsdauer, ggf. die notwendige Pausendauer (Dauerbelastung/Intervallbelastung), aber auch die Belastungshäufigkeit [112]. Bei Patienten, die dies tolerieren, sind mehrere angepasste Gehbelastungen am Tag möglich.

Für sehr schwache und/oder multimorbide Patienten ist das Training mit dem motorgestützten Bewegungstrainer (z. B. dem Motomed ${ }^{\circledR}$ ) (mit oder ohne aktive Unterstützung) eine Option.

Trainingssteuerung und Belastungskontrolle Aufgrund der Pulslosigkeit kann nicht wie üblich die Trainingssteuerung und Belastungskontrolle anhand der Herzfrequenz vorgenommen werden [13]. Für die Trainingssteuerung hat sich die Benutzung der subjektiv vom Patienten empfundenen Belastung durch den Einsatz der BorgSkala (RPE) bewährt. Dabei kann ein Wert $\leq 13$ RPE (,etwas anstrengend") als Orientierung gelten. Bei guter Belastungstoleranz kann die Intensität der Belastung auf einen Wert $\leq 15$ RPE (,,anstrengend") gesteigert werden. Wie bei herztransplantierten Patienten kann zudem die Atemfrequenz über die sogenannte „Sprechregel“ zur Beurteilung der Belastungsintensität herangezogen werden. Die Atemfrequenz sollte dabei eine Unterhaltung erlauben. 
Selbst bei fehlenden leistungsdiagnostischen Daten können diese Parameter bei allen ausdauerorientierten Belastungen zur Belastungsbeurteilung benutzt werden und die Belastung, beginnend auf niedrigem Niveau, allmählich auf die angestrebten Werte gesteigert und angepasst werden.

Das Ergebnis eines 6-Minuten-Gehtests kann zur Einstufung des Gehtrainings dienen. Dabei können die erreichte Gehstrecke, die mittlere Geschwindigkeit sowie die Gehzeit ohne Unterbrechung zur Orientierung für die Trainingssteuerung herangezogen werden [108]. Falls eine spiroergometrische Untersuchung durchgeführt wurde, kann die Intensität bei Dauerbelastungen als Prozentsatz der $\mathrm{VO}_{2 \text { peak }}(40-60 \%)$ oder der $\mathrm{VO}_{2}$ Reserve $\left(\mathrm{VO}_{2} \mathrm{R}\right)(30-50 \%$ oder $\leq 50 \%$ ) [108] festgelegt werden. Liegen Ergebnisse einer Belastungsuntersuchung ohne Spirometrie vor, kann ein Prozentsatz der maximal erreichten Leistung (40-50\% Watt $_{\text {max }}$ ) herangezogen werden. Wenn ermittelbar, ist eine Festlegung der Belastungsintensität an bzw. leicht unterhalb der ventilatorischen Schwelle (VAT1) empfehlenswert. Die Belastungskontrolle während des Trainings erfolgt über die Borg-Skala und die „Sprechregel“.

Während der Rehabilitation sollte das individuell angepasste aerobe Ausdauertraining am besten täglich, ggf. mehrmals täglich in unterschiedlicher Form (Ergometertraining, Gehtraining) durchgeführt werden. Als Trainingsdauer sollten anfangs $>5-10$ min angestrebt werden und diese allmählich den individuellen Möglichkeiten angepasst werden $(\geq 30 \mathrm{~min})$. Bei einem Trainingsfortschritt sollte primär die Belastungsdauer verlängert werden.

Gymnastik Gymnastische Übungen bieten die Möglichkeit, viele Fähigkeiten zu schulen und zu verbessern. Im besonderen Fokus stehen hier Übungen zur Verbesserung der Koordination, insbesondere der Gleichgewichtsfähigkeit, der Flexibilität und Kraft, sowie Übungen, die dazu dienen, das Belastungsempfinden und die Belastungsbeurteilung des Patienten zu schulen. Die Zuordnung zu Kleingruppen mit gymnastischen Übungen kann je nach Zustand des Patienten erfolgen, z. B. in Übungen im Sitzen mit oder ohne Rückenlehne, Kombination von Übungen im Sitzen und Stehen, Kombination von Übungen im Sitzen, Stehen und in Bewegung [112].

Kraftausdauertraining Aufgrund der $\mathrm{zu}$ erwartenden muskulären Dekonditionierung ist anzunehmen, dass VAD-Patienten von einem niedrig bis moderat dosierten dynamischen Kraftausdauertraining profitieren. Nachdem in der rehabilitativen Kardiologie anfänglich Zurückhaltung bestand, wird seit einigen Jahren ein angepasstes Krafttraining bei Patienten mit chronischer Herzinsuffizienz als ergänzende Maßnahme zum aeroben Ausdauertraining empfohlen [119]. Die hierfür publizierten Empfehlungen können als Orientierung für das Training unter Berück- sichtigung der individuellen Gegebenheiten des einzelnen VAD-Patienten und der genannten Sicherheitsaspekte herangezogen werden [12, 119]. Besondere Sorgfalt ist bei der Übungsauswahl und der Wahl der Trainingsgeräte notwendig. Hier können Krafttrainingsgeräte von Vorteil sein, da diese das Bewegungsausmaß und die Bewegungsamplitude einschränken und dadurch die Gefahr inadäquater Übungsdurchführung mit der damit verbundenen Gefahr einer nicht gewollten Belastung der Driveline reduzieren. Auch bei der Übungsauswahl ist dieser Aspekt wichtig. Deshalb sollte auf Übungen für die Bauch- und Rückenmuskulatur verzichtet werden. Vorsicht ist ebenfalls geboten bei allen Übungen für die Muskulatur der oberen Extremitäten und des Schultergürtels.

Der Schwerpunkt sollte deswegen auf dem Training der Muskulatur der unteren Extremitäten liegen. Diese sind entscheidend für die Alltagsbelastbarkeit und zudem von der Dekonditionierung besonders betroffen. Bei einer Thorakotomie sind die oben genannten Einschränkungen zu berücksichtigen. Bei der Durchführung sind zudem abrupte Lagewechsel zu vermeiden. Ein besonderer Wert ist auf eine behutsame individuelle Einführung des Patienten ins Training zu legen. Das Training sollte mit einer sehr niedrigen Intensität begonnen und die Belastung allmählich angepasst werden. Die Belastung sollte mühelos, ohne Ausweichbewegungen und/oder Pressatmung von Patienten durchführbar sein. Als Orientierung kann hier ebenfalls die Borg-Skala herangezogen werden $(\leq 13 \mathrm{RPE}$, bei guter Belastungstoleranz allmähliche Steigerung auf $\leq 15 \mathrm{RPE}$ ). Mit verbessertem Trainingszustand kann das Training den Empfehlungen für Herzinsuffizienzpatienten allmählich angepasst werden [12, 119].

Bei allen Trainingsmaßnahmen sind Elemente der Körperwahrnehmungsschulung zu integrieren. Dies gilt insbesondere für Übungen zur Schulung des Belastungsempfindens und der Belastungsbeurteilung.

\subsection{Ergotherapie während der Rehabilitation von VAD-Patienten}

Die Maßnahmen der Ergotherapie dienen der Wiederherstellung, Entwicklung, Verbesserung, Erhaltung oder Kompensation der krankheitsbedingt gestörten motorischen, sensorischen, psychischen und kognitiven Funktionen und Fähigkeiten [122].

Eine Beratung und Behandlung durch einen Ergotherapeuten mit Kenntnissen und Erfahrungen in der Rehabilitation von VAD-Patienten ist stets erforderlich, wenn diese mittelschwere oder schwere Einschränkungen in den Aktivitäten des täglichen Lebens (ATL) aufweisen (MUST). Diese Patienten sind einer gezielten ergotherapeutischen Befunderhebung $\mathrm{zu}$ unterziehen und sollten einem globalen Assessmentverfahren wie z. B. 
Tab. 10 Ergotherapeutische Maßnahmen bei VAD-Patienten in Abhängigkeit vom funktionellen Status

$\begin{array}{ll}\text { Funktioneller Status/Befund } & \text { Notwendige ergotherapeutische Maßnahmen (MUST) } \\ \text { Mittelschwere bis schwere Defizite } & \text { Training von ATL (z. B. Waschen, Anziehen, Essen) und funktioneller Mobilität zum Umgang mit } \\ \text { in Aktivitäten des täglichen Lebens } & \text { dem VAD (z. B. Akku- und Verbandswechsel), bei Bedarf auch Sensibilitätstraining, Training der } \\ \text { (ATL) } & \begin{array}{l}\text { Auge-Hand-Koordination, Gleichgewichtstraining } \\ \text { Hilfsmittelberatung, Beratung zur Lebensführung mit dem VAD }\end{array} \\ \text { Ftörung der Handmotorik } & \begin{array}{l}\text { Feinmotorik- und Kraftübungen der Hände, Schwerpunkt Fähigkeiten für Device-Handling, Akku- } \\ \text { und Verbandswechsel }\end{array} \\ \text { Kognitive Defizite } & \text { Einüben von Kompensationsstrategien für Aufmerksamkeits-, Lern- und Gedächtnisdefizite }\end{array}$

dem Functional Independence Measure (FIM) [123, 124] zugeführt werden, das ihre Fähigkeiten zur Durchführung der ATL evaluiert. Die Behandlung der Funktionsdefizite wird durch eine ergotherapeutische Beratung zur Lebensführung mit dem VAD ergänzt [125]. Die Frequenz ergotherapeutischer Maßnahmen muss sich an Bedarf und Belastbarkeit orientieren, sie kann von einem einmaligen Assessment mit Hilfsmittelberatung bis hin zu täglichen Therapieeinheiten variieren. Tabelle 10 zeigt, bei welchen Patienten die Ergotherapie während der AHB bei VADPatienten in Abhängigkeit von deren funktionellen Status angezeigt ist.

Bei spezieller Indikation (z. B. bei neurologischen Defiziten, Sehbehinderung, Zuständen nach Amputationen etc.) kommen alle ergotherapeutischen Verfahren in Betracht, die eine Verbesserung von Aktivitäten und Teilhabe im Alltagsleben ermöglichen. Im Fall erschwerter Krankheitsverarbeitung kann die Durchführung bedeutungsvoller und kreativer Tätigkeiten unter ergotherapeutischer Anleitung zur psychischen Stabilisierung beitragen. Bei positiver Prognose für eine Reintegration in das Erwerbsleben kann eine beruflich orientierte Ergotherapie ergänzt werden.

Auch bei der Ergotherapie gelten die im Kapitel Bewegungstherapie beschriebenen Vorsichtsmaßnahmen hinsichtlich Ladezustand der Akkumulatoren, Kreislaufsituation, Zeichen von Infektion, Arrhythmie oder Blutung sowie während der Therapie auftretender Schmerzen, VAD-Alarme oder sonstiger Auffälligkeiten, Sicherung von Driveline und anderen extrakorporalen Komponenten des VAD sowie die Einschränkungen nach frischer Sternotomie.

\subsection{Psychologische Probleme und Lebensqualität sowie deren Erfassung durch Fragebögen bei VAD-Patienten}

Obwohl durch den technologischen Fortschritt in den letzten Jahren die Herzunterstützungssysteme (VAD) kleiner und für den Patienten komfortabler geworden sind, geht ihre Implantation mit einer hohen psychischen Belastung für Patienten und ihre Familien einher. Die permanente Abhängigkeit von einem Gerät, verbunden mit der dauernden Erinnerung an die bestehende Krankheit, das kontinuierliche Monitoring des Geräts und der Blutgerinnung, die Ungewissheit, jederzeit lebensbedrohliche
Komplikationen wie Schlaganfall, Hirnblutungen oder Infektionen erleiden zu können, die oft weiter bestehende allgemeine körperliche Schwäche und die Unsicherheit angesichts des bestehenden Organmangels, eine Herztransplantation $\mathrm{zu}$ erreichen, können $\mathrm{zu}$ verschiedenen psychischen Störungen wie Ängsten, Depressionen oder Anpassungsstörungen führen und die Lebensqualität erheblich einschränken [13, 126, 127]. Das Anpassungsvermögen von Patienten mit einem langjährigen progredienten chronischen Verlauf einer Herzinsuffizienz kann sich dabei von dem anderer Patienten mit einem akuten katastrophalen Ereignis (wie z. B. Herzversagen im Rahmen eines Myokardinfarktes) unterscheiden [13]. Eine posttraumatische Belastungsstörung (PTBS) bei VAD-Patienten und noch häufiger bei nahen Angehörigen scheint aber oft übersehen zu werden und wird selten diagnostiziert [127].

Obwohl gefordert wird, die psychischen Prozesse nach Implantation eines Herzunterstützungssystems sorgfältig $\mathrm{zu}$ evaluieren und $\mathrm{zu}$ behandeln, ist die Studienlage $\mathrm{zu}$ diesem Thema erstaunlich dürftig (Quelle 128 einfügen!), sodass Therapieempfehlungen nur ein niedriges Evidenzniveau erreichen. Berücksichtigung finden sollte auch, dass die meisten Studien zum Thema psychischer Folgen von künstlicher Herzunterstützung bei Patienten mit ,bridge to transplant" und bei pulsatilen Systemen durchgeführt wurden.

Im aktuellen Report des US-amerikanischen INTERMACS-Registers wird bei 1694 Patienten mit nichtpulsatilen VAD im Verlauf eines Jahres eine zunehmende Verbesserung des ,allgemeinen Wohlbefindens“, der „Selbstversorgung“ und der ,gewöhnlichen Alltagsaktivitäten“ als Dimensionen der Lebensqualität berichtet [13]. Auch andere Studien finden eine Verbesserung der gesundheitsbezogenen Lebensqualität bei Patienten nach Implantation eines VAD [64, 103, 129, 130]. Diese scheint unabhängig vom verwendeten Device (pulsatil oder nichtpulsatil) zu sein. Verglichen mit anderen terminal herzinsuffizienten Patienten unter optimaler medikamentöser Therapie berichten Patienten nach Implantation eines VAD über einen besseren Gesundheitszustand, weniger Ängstlichkeit und Depressivität. Sogar im direkten Vergleich mit ihren nicht erkrankten Lebenspartnern schneiden sie in diesen Kategorien besser ab [131]. Deutlich schlechter sind die Ergebnisse hinsichtlich Lebensqualität und psychischer 
Komorbiditäten hingegen im Vergleich zu Transplantatempfängern [131, 132]. In einem systematischen Review zu diesem Thema wird ein Mangel an Studien über den Nutzen einer mechanischen Kreislaufunterstützung aus Patientensicht beklagt [131].

Da die Steigerung der Lebensqualität ein wesentliches Ziel der VAD-Therapie darstellt, wird eine regelmäßige Erfassung der Lebensqualität unter Zuhilfenahme von Fragebögen gefordert [13] (siehe auch Tab. 12). Zumindest am Beginn und Ende einer Rehabilitation sollte der individuelle Nutzen der Behandlung aus Sicht des Patienten thematisiert werden.

Albert et al. [126] untersuchten den Verlauf psychiatrischer Störungen in den ersten 3 Monaten nach Implantation und fanden bei ,älteren“ pulsatilen Systemen mit pneumatischem Antrieb eine rasche Rückbildung „wahnhaft-halluzinatorischer“ und ,ängstlich-depressiver Syndrome" innerhalb der ersten 2 postoperativen Wochen. Andererseits wurde u. a. eine Zunahme eines ,aggressivdysphorischen Syndroms“ und „regressiven Rückzugverhaltens" beobachtet. Eine ,vegetativ-psychomotorische Gespanntheit" blieb während dieser Zeit auf hohem Niveau bestehen. Die Coping-Mechanismen waren vorwiegend charakterisiert durch depressive Bewältigungsstrategien, Verleugnung und Wunschdenken, weniger durch ,aktives problemorientiertes Coping“" [126].

Neurokognitive Störungen in unterschiedlicher Ausprägung treten bei Patienten mit kardialen Erkrankungen in ca. $25-50 \%$ der Fälle auf. Als Ursache werden kardiale, zerebrovaskuläre sowie auch weitere organische und psychosoziale Faktoren diskutiert [133]. Die Prä- valenz neurokognitiver Dysfunktionen bei älteren Herzinsuffizienz-Patienten wird mit ca. 53-58\% angegeben [134]. Bei VAD-Patienten liegen hierzu nur wenige Daten vor. Im INTERMACS-Register hatten $11 \%$ von 5366 Patienten in den ersten 12 Monaten nach Implantation eines VAD einen Schlaganfall erlitten [135]. Da neurologische Ereignisse wie auch Blutungen oder Infektionen unter Herzunterstützung häufiger vorkommen, wird eine neurokognitive Funktionsdiagnostik bei allen VAD-Patienten gefordert [134]. In den Leitlinien [13] wird empfohlen, die neurokognitive Funktion 3, 6, 12 und 18 Monate nach Implantation zu testen. In der Versorgungsrealität wird diese Empfehlung selten umgesetzt. Eine einmalige Testung neurokognitiver Funktionen kann im Rahmen der Rehabilitation zur Identifizierung eines besonderen ergotherapeutischen Förderbedarfs dienen. Neurokognitive Dysfunktionen sind altersabhängig. Da ältere Patienten immer häufiger ein VAD als endgültige Lösung erhalten (destination therapy), erscheinen besonders bei dieser Gruppe regelmäßige Untersuchungen der neurokognitiven Funktion sinnvoll. Die Aussagefähigkeit bisheriger Studien wird zwar durch methodische Mängel eingeschränkt, deren Ergebnisse zeigen jedoch Verbesserungen neurokognitiver Funktionen im Verlauf von 24 Monaten unter künstlicher Herzunterstützung. Dabei fanden sich keine Unterschiede zwischen pulsatilen und kontinuierlichen Systemen [134].

Eine Anschlussheilbehandlung von VAD-Patienten sollte die beschriebenen psychischen Folgezustände berücksichtigen und Unterstützungsmöglichkeiten anbieten. Dabei sollte das gesamte multidisziplinäre Rehabilitationsteam eingebunden sein $[13,114]$. Ein Screening in Form

Tab. 11 Mögliche Aufgabenverteilung im Rehabilitationsteam für eine umfassende psychologische Betreuung von VAD-Patienten

\begin{tabular}{|c|c|c|}
\hline Profession & Diagnostik/Aufgaben & Therapie \\
\hline Arzt & $\begin{array}{l}\text { Wahrnehmung von Hinweisen für psychische Auffälligkeiten } \\
\text { oder mangelnde Adhärenz mit der Behandlung, Erfassung der } \\
\text { individuellen Lebensqualität am Beginn und Ende }\end{array}$ & $\begin{array}{l}\text { Beziehungsaufbau, tägliche Visitengespräche, } \\
\text { Einbezug von Angehörigen }\end{array}$ \\
\hline Psychologe & $\begin{array}{l}\text { Screening auf psychosoziale Belastung durch diagnostische Ge- } \\
\text { spräche, Fragebögen zur Selbstbeurteilung, Testauswertung }\end{array}$ & $\begin{array}{l}\text { Psychoedukation, Stressbewältigungs- und } \\
\text { Entspannungstraining }\end{array}$ \\
\hline $\begin{array}{l}\text { Approbierter } \\
\text { Psychotherapeut }\end{array}$ & $\begin{array}{l}\text { Diagnosestellung psychischer Störungen (z. B. Angst, } \\
\text { Depression) }\end{array}$ & Unterstützende Gespräche, Verhaltenstherapie \\
\hline $\begin{array}{l}\text { Ggf. Facharzt für } \\
\text { Psychiatrie oder psycho- } \\
\text { somatische Medizin }\end{array}$ & V. a. Suizidalität, neurokognitive Defizite, Psychose & $\begin{array}{l}\text { Psychopharmakologie, Verlegung in } \\
\text { Akutversorgung }\end{array}$ \\
\hline $\begin{array}{l}\text { Neuropsychologe, } \\
\text { medizinische Fachan- } \\
\text { gestellte (MFA) oder } \\
\text { psycholog. techn. } \\
\text { Assistentin (PTA) }\end{array}$ & $\begin{array}{l}\text { V. a. neurokognitive Defizite, diagnostische Fragebögen, neuro- } \\
\text { psychologische Testung, Testauswertung }\end{array}$ & Computerassistierte Therapie \\
\hline Pflegedienst & $\begin{array}{l}\text { Screening auf psychiatrische Symptome, Hinweise für Ver- } \\
\text { haltensauffälligkeiten im Stationsalltag }\end{array}$ & $\begin{array}{l}\text { Unterstützung bei Krankheitsverarbeitung, } \\
\text { Vermittlung von Sicherheit mit dem Gerät, } \\
\text { Verbänden und dem INR-Monitoring }\end{array}$ \\
\hline Sozialdienst & $\begin{array}{l}\text { Information über Hilfsmöglichkeiten, Klärung der häuslichen } \\
\text { psychosozialen Situation und der Weiterbetreuung }\end{array}$ & $\begin{array}{l}\text { Information, Gespräche, Einbezug von } \\
\text { Angehörigen }\end{array}$ \\
\hline Ergotherapie & $\begin{array}{l}\text { Diagnostik von neurologischen Defiziten und Einschränkungen } \\
\text { bei Aktivitäten des täglichen Lebens }\end{array}$ & Spezialisiertes Training \\
\hline
\end{tabular}


Tab. 12 Empfohlene Fragebögen für das psychologische Screening und Assessment im Rahmen der Anschlussheilbehandlung von VAD-Patienten (verwendet auch bei $[61,64,103,130,131,136]$ )

\begin{tabular}{llc}
\hline $\begin{array}{l}\text { HADS [137] } \\
\text { Generisch }\end{array}$ & Ängstlichkeit und Depressivität & Benötigte Zeit (min) \\
SF 36 [138] & $\begin{array}{l}\text { Körperliche Funktionsfähigkeit und Rollenfunktion, Schmerzen, Gesundheits- } \\
\text { wahrnehmung, Vitalität, Soziale Funktionsfähigkeit, emotionale Rollenerfüllung, } \\
\text { psychisches Wohlbefinden }\end{array}$ & 5 \\
EuroQol [139, 140] & Mobilität, Selbstversorgung, übliche Aktivitäten, Schmerz, Depressivität & $1-2$ \\
Krankheitsspezifisch & Körperliche Einschränkung, Symptome (Häufigkeit und Schwere), Selbstwirksam- & $4-6$ \\
KCCQ [141, 142] & keit, soziale Einschränkungen, psych. Lebensqualität. & $5-10$ \\
KLHFQ [143,144] & Körperliche, emotionale Einschränkung & 5
\end{tabular}

von Selbstbeurteilungsfragebögen oder durch psychosomatisch geschulte Ärzte oder Psychologen erscheint daher zu Beginn der Rehabilitation sinnvoll. Es sollte dabei besonders berücksichtigt werden, dass VAD-Patienten sich während der Rehabilitation häufig in einer „Honeymoon“Phase mit euphorischer Grundstimmung befinden [13]. Eine wichtige und herausfordernde Aufgabe der AHB ist es, die Patienten auf die kommenden Belastungen in ihrer gewohnten Umgebung vorzubereiten. Tabelle 11 zeigt eine mögliche Aufgabenverteilung im Rehabilitationsteam für eine umfassende psychologische und neuropsychologische Betreuung von VAD-Patienten. In der nachfolgenden Tab. 12 sind empfohlene Assessmentverfahren zusammengefasst.

\subsection{Besonderheiten bei der Ernährung von VAD- Patienten}

Der Ernährungsstatus hat einen wesentlichen Einfluss auf das Überleben und die Lebensqualität bei Patienten mit mechanischer Kreislaufunterstützung [145]. Ein reduzierter Ernährungszustand (auch als Dystrophie bezeichnet), zeigt sich durch ungenügend vorhandenes subkutanes Fettgewebe mit Hypalbuminämie, vermindertem Gesamteiweiß und reduzierten Lymphozyten. Durch eine Apoptose der Lymphozyten, verursacht durch den Kontakt mit biosynthetischen Materialien, kommt es zu einem Defekt der zellulären Immunität mit erhöhter Infektionsrate und prolongierter Wundheilung insbesondere bei älteren VADPatienten [146, 147]. Weiterhin ist ein niedriges Körpergewicht mit einer erhöhten Sepsisrate und einer erhöhten Mortalität verknüpft (s. u.), weshalb die BMI-Kontrolle als wichtige Verlaufsbeobachtung zu bewerten ist [148].

Ein höherer Energieverbrauch führt bei chronisch herzinsuffizienten Patienten häufig zu einer Gewichtsreduktion, für die der Begriff kardiale Kachexie geprägt wurde. Betroffen sind 10-15\% der Patienten mit chronischer Herzinsuffizienz, insbesondere jene mit reduzierter linksventrikulärer Ejektionsfraktion [49].

Unter Kachexie wird ein komplexes StoffwechselSyndrom mit unbeabsichtigter Gewichtsreduktion von $\geq 5 \%$ der Gesamtkörpermasse innerhalb von 12 Monaten verstanden, die nicht auf einer Ödem-Beseitigung basiert. Obligat ist der Muskelmasseverlust, fakultativ der (zusätzliche) Fettgewebsverlust.

Zusätzlich sollen 3 weitere der folgenden Kriterien erfüllt sein [149]:

1. Reduzierte Muskelkraft (niedrigstes Perzentil)

2. Fatigue (Erschöpfung)

3. Anorexie

4. Niedrige freie Fettmasse

5. Pathologisches Labor:

- $\operatorname{CRP}(>5,0 \mathrm{mg} / \mathrm{l})$

- Anämie $(<12 \mathrm{~g} / \mathrm{dl})$

- Hypalbuminämie $(<3,2 \mathrm{~g} / \mathrm{dl})$

Die Prädilektionsstellen des kardial bedingten Gewebeverlusts sind die unteren Extremitäten, auch wegen der Inaktivitätsatrophie [149]. Zurückzuführen ist die Kachexie zusätzlich auf einen reduzierten Appetit und eine sekundär erhöhte Zytokinkonzentration, z. B. des TNF. Außerdem kommen verminderte Belastbarkeit, erhöhte Atemarbeit sowie verfrühtes Sättigungsgefühl durch Hepatomegalie und Darmödem hinzu [150]. Zudem zeigt sich, dass eine früh-postoperative und aggressive Kalorienzufuhr kritisch zu bewerten ist [151-153]. Bei manifester chronischer Herzinsuffizienz kommt es auch zu einem Mangel an Vitaminen und Spurenelementen einschließlich Vitamin C, D, Magnesium und Zink. Eine Supplementierung des Fehlenden führt zu einer besseren Prognose [154].

Die Hypalbuminämie (Serum-Albumin <3,3 $\mathrm{mg} / \mathrm{dl}$ ) wurde als ein bedeutender Risikofaktor identifiziert, der die Sterblichkeit bei VAD-Patienten mit langfristiger Unterstützungstherapie um den Faktor 6,6 erhöht [145]. 


\section{Diagnostik bei Rehabilitationsbeginn}

Basisdiagnostik Ernährungsstatus (MUST) Gekennzeichnet wird der Ernährungsstatus bei Rehabilitationsbeginn durch folgende Daten:

1. Größe, Gewicht, Bauchumfang

2. BMI

3. Albumin (MUST) und Präalbumin (COULD) im Serum

4. Gesamtcholesterin

5. Lymphozytenzahl

Relevant für die Einschätzung einer Malnutrition (bei VADPatienten als auch anderen Patienten) sind folgende Werte: Albumin $<3,2 \mathrm{mg} / \mathrm{dl}$, Präalbumin $<16 \mathrm{mg} / \mathrm{dl}$, Gesamtcholesterin $<130 \mathrm{mg} / \mathrm{dl}$, Lymphozyten $<100 / \mu \mathrm{l}$ [155]. Präalbumin verhält sich indirekt proportional zum CRP. Bei Entzündungen sind ein erhöhtes CRP und ein vermindertes Präalbumin zu erwarten.

Folgende weitere biochemische Marker, die auf eine Malnutrition hinweisen, sind beschrieben worden: Transferrin, Retinol-bindendes Protein, CRP [151, 153, 156, 157].

Mini Nutritional Assessment (MNA ${ }^{\circledR}$ ) (COULD) Ein schnelles klinisches Screening gelingt mit Hilfe des evaluierten Fragebogens MNA ${ }^{\circledR}$, welcher als PDF-Vorlage aus dem Internet zur Verfügung steht und online ausgefüllt werden kann. Er existiert sowohl in der Lang- als auch in der ebenfalls validierten Kurzform (Abb. 7). Letztere wird zur Anwendung empfohlen [158]. Anhand von fünf Fragen (A-E) und der Feststellung des Körpermasseindex und des Wadenumfangs ist eine vorläufige Diagnose zu stellen. Der Körpermasseindex gibt Hinweise auf den Grad von Überoder Untergewicht, der Umfang der Wadenmuskulatur spiegelt eine Sarkopenie wider, wenn er unter $31 \mathrm{~cm}$ liegt.

\section{Therapie}

Die amerikanischen Leitlinien-Empfehlungen der ISHLT beziehen sich auf den Ernährungsstatus vor der VAD-Implantation [13], Hinweise für die Zeit danach enthalten sie nicht.

Flüssigkeitsmanagement (MUST) In der Regel ist die Herzinsuffizienz nach einer VAD-Implantation weitestgehend kompensiert. Daher müssen vorherige Auflagen bzgl. Flüssigkeitsrestriktion in der Regel nicht weitergeführt werden. Wenn dennoch schwere Symptome persistieren, insbesondere einer Insuffizienz des nicht unterstützten rechten Ventrikels, kann eine Flüssigkeitsrestriktion auf 1,5-2 $1 \mathrm{~d}$ notwendig sein [49].

Ernährungsberatung (MUST) VAD-Patienten sollten nach ärztlicher Festlegung der Kostform grundsätzlich in die Ernährungsberatung eingeplant werden: Weisen klinische Untersuchungen und ggf. der MNA ${ }^{\circledR}$-Test (Abb. 7) auf eine Malnutrition oder Kachexie hin, wird neben der Labordiagnostik Einzel-Ernährungsberatung verordnet. Aus der Laboranalyse ergeben sich ggf. weitere ärztliche Verordnungen. Die Kostform wird in Absprache mit dem Patienten festgelegt. Dabei sind auch Begleiterkrankungen zu berücksichtigen, etwa Stoffwechselstörungen (Diabetes mellitus, Hyperurikämie). Die Regelkostform orientiert sich an den Nährstoffempfehlungen der DGE [159].

\section{Ernährung bei Untergewichtigen}

Anabolika In der ESC-Leitlinie [49] zur chronischen Herzinsuffizienz wird unter Hinweis auf fehlende Evidenz von Appetit-Stimulanzien, Anabolika (Insulin, anabole Steroide) in Kombination mit supplementärer Ernährung abgeraten [153]. Bei anderen Erkrankungen, die ebenfalls zur Kachexie führen können, z. B. der COPD, war ihr Einsatz erfolgreich [160].

Wunschkost Primär wird das Ziel der Gewichtszunahme verfolgt, dem andere Aspekte, z. B. ein ,ungesunder“" Fettund Zuckergehalt der Nahrung, unterzuordnen sind. Hier wird eine Wunschkost eingesetzt, bis eine signifikante Gewichtszunahme, mindestens bis zum Normgewicht, erreicht wird.

Formuladiäten Bei erwiesener Malnutrition können Formuladiäten mit kalorienreichen Flüssignahrungen eingesetzt werden. Als zusätzliche Möglichkeit kann auch eine enterale Ernährungssonde für die Zuführung hyperkalorischer Nährlosung erwogen werden.

PEG In seltenen Fällen, wenn eine adäquate orale Nahrungsaufnahme nicht mehr sichergestellt werden kann, ist die Anlage einer perkutanen endoskopisch kontrollierten Gastrostomie (PEG) erforderlich, Diese ist insbesondere bei absehbarer längerfristiger Gabe einer Sondenernährung statt einer nasogastralen oder nasoduodenalen Sonde $\mathrm{zu}$ empfehlen [161].

\section{Übergewicht, Adipositas}

Obesitas-Paradoxon Die Adipositas ist in ihrer milden Ausprägung (BMI $30-35 \mathrm{~kg} / \mathrm{m}^{2}$ ) bei chronischer Herzinsuffizienz mit einem Überlebensvorteil und einer niedrigeren Hospitalisierungsrate im Vergleich zu Normalgewichtigen verknüpft [162], wofür der Begriff ObesitasParadoxon Verwendung findet. Die Validität eines Obesitas-Paradoxons wird in der US-amerikanischen Herzinsuffizienz-Leitlinie angezweifelt [163], stattdessen wird vielmehr eine U-förmige Kurve für den BMI postuliert 
Abb. 7 Kurzversion des Mini Nutritional Assessment - MNA ${ }^{\circledR}$ zur Einschätzung des Ernährungszustands [158]

\section{Mini Nutritional Assessment MNA $^{\circledR}$ \\ Nestlé NutritionInstitute

\begin{tabular}{|c|c|c|c|c|}
\hline Name: & & & Vorname: & \\
\hline Geschlecht: & Alter (Jahre) & Gewicht $(\mathrm{kg})$ : & Größe $(\mathrm{cm})$ : & Datum: \\
\hline
\end{tabular}

Füllen Sie den Bogen aus, indem Sie die zutreffenden Zahlen in die Kăstchen eintragen. Addieren Sie die Zahlen, um das Ergebnis des Screenings zu erhalten.

\section{Screening}

A Hat der Patient während der letzten 3 Monate wegen Appetitverlust, Verdauungsproblemen, Schwierigkeiten beim Kauen oder Schlucken weniger gegessen?

$0=$ starke Abnahme der Nahrungsaufnahme

$1=$ leichte Abnahme der Nahrungsaufnahme

2 = keine Abnahme der Nahrungsaufnahme

B Gewichtsverlust in den letzten 3 Monaten

$0=$ Gewichtsverlust $>3 \mathrm{~kg}$

$1=$ nicht bekannt

2 = Gewichtsverlust zwischen 1 und $3 \mathrm{~kg}$

$3=$ kein Gewichtsverlust

C Mobilität

$0=$ bettlägerig oder in einem Stuhl mobilisiert

$1=$ in der Lage, sich in der Wohnung zu bewegen

2 = verlässt die Wohnung

D Akute Krankheit oder psychischer Stress während der letzten 3 Monate? $0=$ ja $\quad 2=$ nein

E Neuropsychologische Probleme $0=$ schwere Demenz oder Depression $1=$ leichte Demenz

2 = keine psychologischen Probleme

F1 Body Mass Index (BMI): Körpergewicht (kg) / Körpergröße $\left.{ }^{2}\left(\mathrm{~m}^{2}\right)\right)$ $0=\mathrm{BMI}<19$

$1=19 \leq \mathrm{BMI}<21$

$2=21 \leq B M I<23$

$3=\mathrm{BMI} \geq 23$

WENN KEIN BMI-WERT VORLIEGT, BITTE FRAGE F1 MIT FRAGE F2 ERSETZEN. WENN FRAGE F1 BEREITS BEANTWORTET WURDE, FRAGE F2 BITTE OBERSPRINGEN

F2 Wadenumfang (WU in $\mathrm{cm}$ )

$0=W U<31$

$3=W U \geq 31$

Ergebnis des Screenings

(max. 14 Punkte)

12-14 Punkte: $\square$ Normaler Ernährungszustand

8-11 Punkte: $\quad \square$ Risiko für Mangelernährung

0-7 Punkte:

Mangelernährung

Für ein tiefergehendes Assessment ( $\leq 11$ Punkte), bitte die vollständige Version des $\mathrm{MNA}^{\circ}$ ausfüllen, die unter www.mna-elderly.com zu finden ist.

Wurde das Screening mit Beantwortung der Frage F2 (Wadenumfang) durchgefürt, ist die MNA ${ }^{\bullet}$ - Long Form für ein tiefer gehendes Assessment nicht geeignet, bei Bedarf ein anderes Assessment (z.B. PEMU) durchführen.

Ref. Vellas B, Villars H, Abellan G, et al. Overview of the MNA@ - Its History and Challenges. J Nutr Health Aging 2006;10:456-465. Rubenstein LZ, Harker JO, Salva A, Guigoz Y, Vellas B. Screening for Undernutrition in Geriatric Practice: Developing the Short-Form Min Nutritional Assessment (MNA-SF). J. Geront 2001;56A: M366-377.

Guigoz Y. The Mini-Nutritional Assessment (MNA) Review of the Literature - What does it tell us? J Nutr Health Aging 2006; 10:466-487. Kaiser MJ, Bauer JM, Ramsch C, et al. Validation of the Mini Nutritional Assessment Short-Form (MNAQ-SF): A practical tool for identification of nutritional status. J Nutr Health Aging 2009; 13:782-788.

(B) Société des Produits Nestlé, S.A., Vevey, Switzerland, Trademark Owners

O Nestlé, 1994, Revision 2009. N67200 12/99 10M

Mehr Informationen unter: www.mna-elderly.com 
Tab. 13 Ernährungsempfehlungen für normalgewichtige und adipöse VAD-Patienten nach Montgomery et al. [155]

\begin{tabular}{lll} 
& Normalgewicht & Adipositas \\
\hline Energiebedarf & $30-35 \mathrm{kcal} / \mathrm{kg}$ oder Grundumsatz $+15-25 \%$ & $\begin{array}{l}21 \mathrm{kcal} / \mathrm{kg} \text { (bezogen auf das aktuelle Gewicht, } \\
\text { indirekte Kalorimetrie, spiroergometrisch) }\end{array}$ \\
Eiweißbedarf & für minimale physische Aktivität & $1,5-2 \mathrm{~g} / \mathrm{kg}$ des Idealgewichts \\
$\mathrm{Na}^{+}$ & $1-1,5 \mathrm{~g} / \mathrm{kg}$ & \\
Flüssigkeit & $2 \mathrm{~g} \mathrm{Natrium} / \mathrm{d}$ & \\
Natürliche Ernährung & $1,5-21$ & \\
Enterale Ernährung & Kleine, häufige Mahlzeiten, ausreichend einweißreich \\
Supplementierung: Vitamine, Mineralien & Dünndarmsonde erwägen: konzentrierte hypoosmolare, ballaststofffreie Formuladiäten \\
& Vitamin B: bei nachgewiesenem Mangel bzw. bei mangelndem Appetit und unzureichender oraler \\
& Nahrungsaufnahme \\
& Vitamin D: bei nachgewiesenem Defizit \\
& Zink: bei nachgewiesenem Defizit \\
\hline
\end{tabular}

[164]. Die Mortalität ist bei Kachexie am höchsten, niedriger bei Normalgewicht, Übergewicht und milder Adipositas, sie ist gesteigert bei höhergradiger Adipositas.

Ein U-Kurven-Phänomen ist auch bei VAD-Patienten nachgewiesen worden, zumindest bis 30 Tage nach Implantation: hier war die Mortalität bei Übergewichtigen (BMI 25-29 kg/ $\mathrm{m}^{2}$ ) am geringsten. Lag der BMI unter 20 oder über $35 \mathrm{~kg} /$ $\mathrm{m}^{2}$, bestand das höchste Mortalitätsrisiko. Geringfügig benachteiligt waren VAD-Patienten mit einem BMI von 20-24 kg/m² im Vergleich zu leicht Adipösen mit einem BMI von $30-34 \mathrm{~kg} / \mathrm{m}^{2}$. Akzentuiert war die schlechtere Prognose bei höherem Alter, biventrikulärer Implantation und Kardiomyopathie als Primärdiagnose [165].

\section{Adipositas $\left(B M I \geq 30 \mathrm{~kg} / \mathrm{m}^{2}\right)$}

Gewichtsabnahme (SHOULD) Der Nutzen einer Gewichtsreduktion ist vor dem Hintergrund der Diskussion über das Obesitas-Paradoxon bei VAD-Patienten unklar. Begleiterkrankungen sind für die Entscheidung zu berücksichtigen. Folgende Ziele können innerhalb von 6 bis 12 Monaten sinnvoll sein:

- BMI 30 bis $<35 \mathrm{~kg} / \mathrm{m}^{2}$ : keine Reduktion oder Reduktion um $>5 \%$ des Ausgangsgewichts, wenn Begleiterkrankungen dieses erfordern

- BMI >35 kg/m²: Reduktion um $>10 \%$ des Ausgangsgewichts

In dieser Situation wird auf die deutsche S-3-Leitlinie zur Prävention und Therapie der Adipositas verwiesen [159].

Ernährungsempfehlungen für VAD-Patienten bei Normalgewicht und Adipositas können der Tab. 13 entnommen werden.

\subsection{Schulung von Patienten: Aufgaben des Rehabilitationsteams}

Die Systemkomponenten eines VAD müssen vom Patienten sicher und fehlerfrei gehandhabt werden. Vom Selbstmanagement hängt der Langzeiterfolg der VAD-Therapie entscheidend ab [145]. Das besondere Verhältnis zwischen dem VAD und seinen ,Trägern“ bedeutet eine große Herausforderung für die VAD-Patienten, deren Angehörige und die betreuenden Behandler im Rehabilitationsteam. Während der Anschlussheilbehandlung soll durch Behandlung und Schulung die erfolgreiche selbstständige Fortsetzung der VAD-Therapie im privaten Umfeld vorbereitet werden [28].

Zusammen mit den Patienten sollten zum Beginn der Rehabilitationsmaßnahme die für die Schulung und Information wesentlichen Ziele besprochen und hinsichtlich der Priorität in eine Reihenfolge gebracht werden. Im Rahmen eines multimodalen Therapieansatzes sollten die Berufsgruppen des Rehabilitationsteams in regelmäßiger Abstimmung die verschiedenen Schulungsthemen ansprechen und vermitteln. Da der Therapieerfolg in erster Linie vom korrekten Umgang mit dem System abhängt, gehört diesem Thema oberste Priorität.

Je nach aktuellem Aufnahmevermögen der Patienten und ggf. seiner Angehörigen oder Lebenspartner kann es während der AHB angezeigt sein, die weiteren Themen nur ansatzweise oder in Teilen anzusprechen. Eine Fortsetzung der Schulung und Information kann nach langer akutmedizinischer Behandlung in einer weiteren Rehabilitationsmaßnahme zeitversetzt notwendig sein. Dadurch kann bei älteren Patienten die Selbstständigkeit und Teilhabe im Alltagsleben erhöht und bei jüngeren Patienten die Reintegration ins Berufsleben unterstützt werden.

\section{Schulungsthemen}

Umgang mit dem VAD-System Nach dem Wechsel aus der akutmedizinischen Behandlung in die Rehabilitationsklinik steht die sichere Handhabung von Akkus und Ladegerät im Vordergrund, da Fehler bei der Stromversorgung des VAD fatale Folgen haben können [61].

Ein weiteres Schulungsthema ist der Umgang mit dem Controller. Die Bedeutung der verschiedenen Systemalarme und die daraus abzuleitenden Reaktionen müssen sicher beherrscht werden und sind damit wichtiger Bestandteil der Patientenschulung. Ein Controllerwechsel sollte schon 
in der implantierenden Akutklinik demonstriert oder durchgeführt worden sein. In der Rehabilitationsklinik wird dies in der Regel nur noch theoretisch besprochen. Ein Controllerwechsel durch den Patienten allein sollte nicht stattfinden, da währenddessen mit einem Bewusstseinsverlust gerechnet werden muss. Aus diesem Grunde kommt die Schulung eines Controllerwechsels am ehesten für den Patienten zusammen mit Angehörigen in Frage. Zum besseren Verständnis des Controllers soll der Patient die im Display angezeigten Werte einmal täglich dokumentieren. Dadurch lernt er die Funktionswerte $\mathrm{zu}$ beobachten und $\mathrm{zu}$ interpretieren, um somit bei Auffälligkeiten selbstständig reagieren zu können. Je besser der Patient die Funktionswerte und Funktionsweise des VAD versteht, umso höher werden seine Akzeptanz und sein Verständnis für die erforderlichen begleitenden Therapien wie z. B. die Antikoagulation sein. Da eine Abfrage des VAD-Datenspeichers häufig nur im implantierenden Zentrum möglich ist, stellt die selbstständige Dokumentation der Funktionswerte eine wichtige Informationsquelle für die weiterbehandelnden Ärzte und Pflegedienste dar. Auch für Rücksprachen mit dem implantierenden Zentrum ist die Angabe der Funktionsparameter eine wichtige Grundlage.

Die Versorgung der Eintrittsstelle der Driveline ist prioritärer Schulungsinhalt für die Patienten und deren Angehörige und ggf. auch für externes Pflegepersonal [166]. Die erforderlichen Verbandswechsel sind entsprechend den Standards des implantierenden Zentrums zu schulen. Ziel ist die Befähigung der Patienten und seiner Angehörigen, diese zu Hause selbstständig durchführen zu können.

Die Qualität des INR-Managements bestimmt maßgeblich den störungsfreien Verlauf der eingesetzten Aggregate. Basis für die Patientenschulung sind die standardisierten Vorgaben der ASA (Arbeitsgemeinschaft Selbstkontrolle der Antikoagulation e. V.) [167]. Wünschenswert ist eine Ausrüstung des Patienten mit eigenem Messgerät bereits in der Akutklinik. Besonders zu beachten sind Vorgaben des implantierenden Zentrums bzgl. eines Bridgings der Antikoagulation bei Unterschreiten des Zielbereichs und bei notwendigen operativen Eingriffen. Die Patienten sollten geschult werden, Zeichen einer gastrointestinalen Blutung frühzeitig zu erkennen. Die Notwendigkeit einer raschen Abklärung im Verdachtsfall muss vermittelt werden [168].

Die NOAK, deren Wirkungsmechanismus auf dem Vitamin-K-Antagonismus beruht, werden an dieser Stelle nicht besprochen, da sie für den Einsatz bei VAD-Patienten noch nicht zugelassen sind.

Behandlung der Herzinsuffizienz Ein wichtiges Schulungsziel ist die Kenntnis der Wirkstoffe sowie der typischen Wirkungen und Nebenwirkungen der aktuell eingesetzten Medikation. Das Pflegeteam unterrichtet den Patienten im sicheren Dosieren und Einnehmen der Medikamente. Auch soll der Patient darauf hingewiesen werden, bei größeren Gewichtsveränderungen die Trinkmenge bzw. die Diuretika-Therapie anzupassen.

Wenn eine orientierende Messung des mittleren Blutdrucks mit einem Oberarm-Blutdruckmessgerät möglich ist, sollte der Patient darin geschult werden. Den Patienten muss ein regelmäßiges Eintragen der täglichen Vitalzeichenkontrollen vermittelt werden, sodass sie selbst, ihre Hausärzte und die VAD-Ambulanz des implantierenden Zentrums diese Werte zur Therapiekontrolle nutzen können [169]. Die Bedeutung des verordneten bewegungstherapeutischen Programms muss vermittelt werden.

Präventiver Lebensstil Die Themen Ernährung, Nikotin/ Alkohol und Stressmanagement sollten angesprochen werden, wobei die Notwendigkeit und der Umfang diesbezüglicher Schulungen von der individuellen Gesundheitssituation der Patienten abhängen. Zum Abschluss der AHB stehen die Vermittlung individualisierter Ziele für Schutz- und Risikofaktoren und die Belastbarkeit im Alltag im Vordergrund.

Beratung zur Teilhabe am Alltagsleben Schulungen zur Teilhabe am Alltagsleben, u. a. auch zur finanziellen Absicherung, sind in den allgemeinen Programmen der Rehabilitationskliniken enthalten. Auf die besonderen Bedürfnisse von VAD-Patienten ist durch individuelle Beratung einzugehen. Auch eine Beratung zur sexuellen Aktivität eines Patienten mit VAD kann individuell

Tab. 14 Die wichtigsten Schulungsinhalte und deren Vermittlung durch das Rehabilitationsteam bei einer Anschlussheilbehandlung von VADPatienten

\begin{tabular}{llll} 
Schulungsthemen & Inhalte & $\begin{array}{l}\text { Vermittelt durch folgende } \\
\text { Berufsgruppen }\end{array}$ & MoSCoW \\
\hline Umgang mit dem VAD-System & $\begin{array}{l}\text { Stromversorgung, Controller, Anti- } \\
\text { koagulation, Driveline-Versorgung } \\
\text { Medikamente, Vitalzeichenkontrolle, } \\
\text { Training }\end{array}$ & $\begin{array}{l}\text { Ärzte, Pflegekräfte, } \\
\text { Kardiotechniker }\end{array}$ & $\begin{array}{l}\text { Ärzte, Bewegungstherapeuten, } \\
\text { Pflegekräfte }\end{array}$ \\
& $\begin{array}{l}\text { Ernährung, Nikotin/Alkohol, } \\
\text { Präventiver Lebensstil }\end{array}$ & $\begin{array}{l}\text { Bewegungstherapeuten, Er- } \\
\text { nährungsberater, Pflegekräfte, }\end{array}$ & SHOULD \\
Stressmanagement & $\begin{array}{l}\text { Ärzte, Psychologen } \\
\text { Sozialarbeiter, Ärzte, Pflege- } \\
\text { kräfte, Psychologen }\end{array}$ & SHOULD \\
& $\begin{array}{l}\text { Private und ggf. berufliche Integration, } \\
\text { häusliche Versorgung }\end{array}$ & &
\end{tabular}


Abb. 8 Exemplarisches Beispiel für einen Patientenführerschein, Seite 1/2 [170]

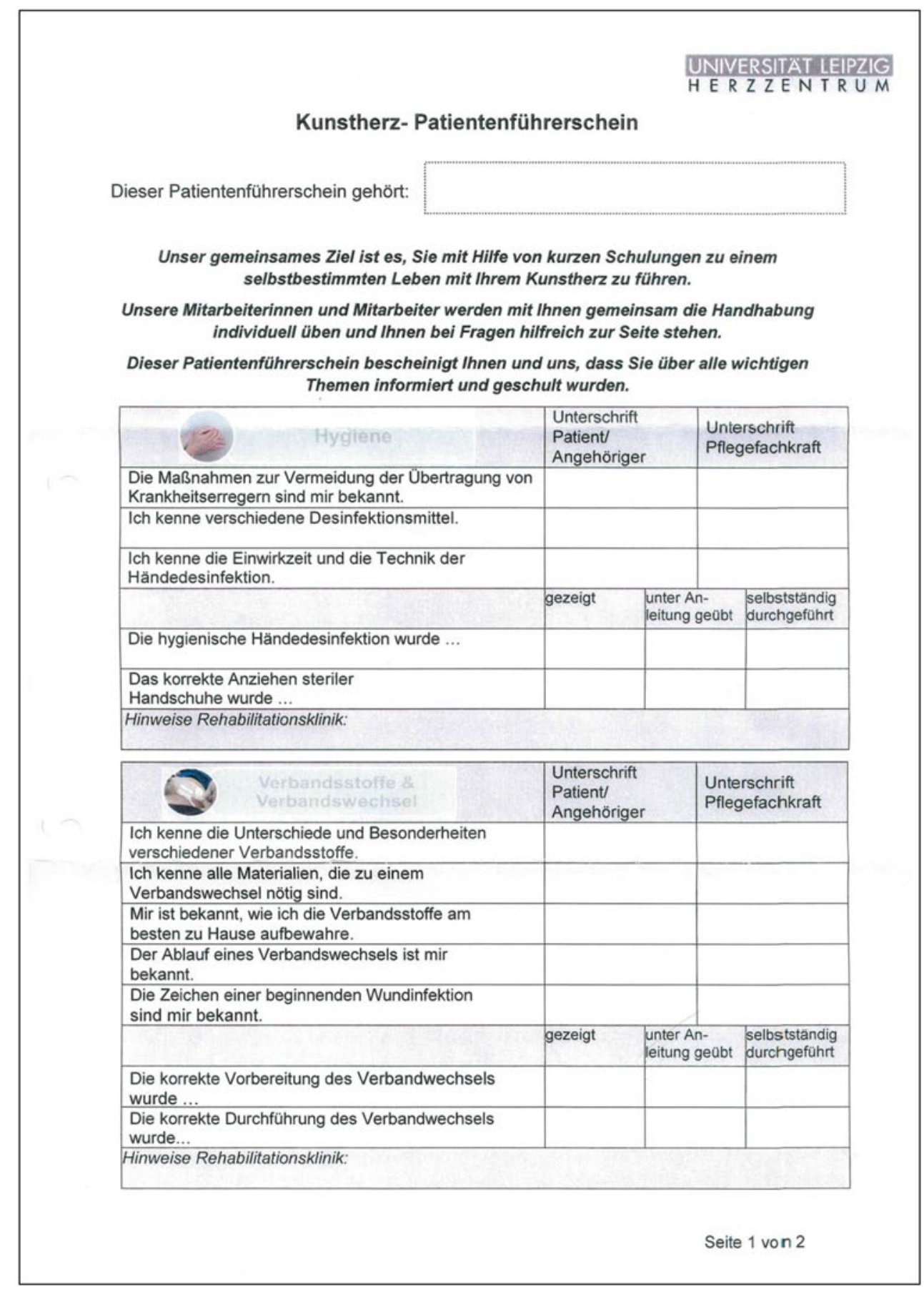

durch Ärzte und/oder Psychologen durchgeführt werden. Informationen zur Durchführung von Reisen und selbstständigem Führen von Fahrzeugen etc. werden gemäß den in Abschn. 2.15 dargelegten Prinzipien vermittelt.

In Tab. 14 sind die für VAD-Patienten wichtigsten Schulungsthemen, die während einer Anschlussheilbehandlung vermittelt werden, zusammengefasst.

Eine gute Orientierung für die Schulungsinhalte und die Dokumentation des erreichten Kenntnisstands bietet ein sogenannter Patientenführerschein (siehe Abb. 8 und 9).

\subsection{Sozialarbeit und Entlassungsmanagement der Rehabilitationskliniken sowie Beratungsgrundlagen für Arbeiten, Fahren und Reisen mit einem VAD}

Sozialmedizinische Beurteilung/Beratung Vor Ende der Rehabilitation stellt sich auch bei einem VAD-Patienten die Frage der sozialmedizinischen Beurteilung und damit auch der Leistungseinschätzung bezogen auf eine weitere berufliche Tätigkeit. Dabei sollten folgende Themen- 
Abb. 9 Exemplarisches Beispiel für einen Patientenführerschein, Seite 2/2 [170]

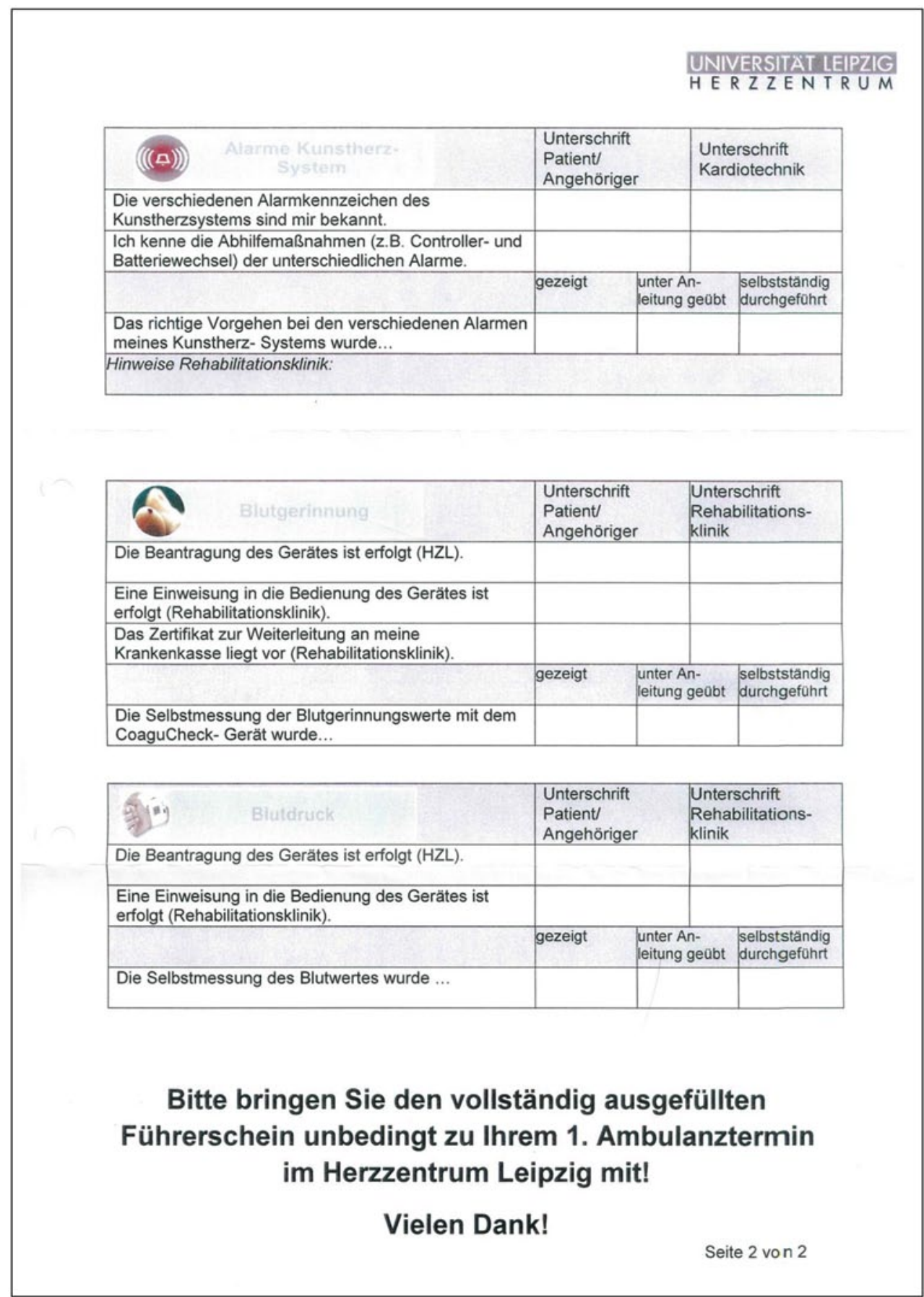

bereiche erörtert werden: Selbstversorgung, Bewältigung des häuslichen Lebens, Arbeit und Beschäftigung sowie wirtschaftliche Sicherstellung. Je nach Bedarf müssen dann Maßnahmen zur Versorgung für die Zeit nach der Entlassung empfohlen und/oder eingeleitet werden. Des Weiteren sollte für einen vorübergehenden Zeitraum eine Nachsorge durch einen Sozialarbeiter angeboten werden.

Bei Patienten mit einem mechanischen Herzunterstützungssystem sollte vor dem Hintergrund der schweren Grunderkrankung eine aufgehobene Erwerbsfähigkeit die Begutachtung prägen. In der Regel sollte die tägliche Arbeitszeit eines VAD-Patienten die Grenze von $3 \mathrm{~h}$ nicht überschreiten. Die Beurteilung sollte den Antrag auf eine Erwerbsminderungsrente ermöglichen.

Das heißt allerdings im Umkehrschluss nicht, dass ein Patient mit einem VAD nicht arbeiten könnte. Ermöglicht ein berufliches Wiedereingliederungsmanagement im Angestelltenverhältnis oder eine selbstständige Tätigkeit eine an die Grunderkrankung angepasste Arbeit mit überwiegend leichten körperlichen Tätigkeiten, so ist 
diese bei entsprechendem Wunsch des Patienten auch vorstellbar. Im Falle zusätzlicher Implantate (ICD/CRT etc.) sollten am Arbeitsplatz elektromagnetische Interferenzen, welche Fernfeldwahrnehmungen induzieren könnten, vermieden werden. Auch das VAD selbst sollte keinen starken Temperaturbelastungen oder elektromagnetischen Interferenzen ausgesetzt werden [11, 61].

Bei der Beurteilung sollten die speziellen Erfordernisse des VAD-Systems mit der jeweiligen Arbeitsplatzsituation abgeglichen werden. Hier liegen $u$. a. auch die Begründungen für die aufgehobene Leistungsfähigkeit im Erwerbsleben. Weitere technische Entwicklungen wie die induktive Energieübertragung und Miniaturisierung der Geräte werden in Zukunft möglicherweise zu einer Veränderung der sozialmedizinischen Begutachtung beitragen.

\section{Entlassungsmanagement}

Die Entlassung ins häusliche Umfeld ist von entscheidender Bedeutung für den Patienten und seine Familie. Nachdem der Patient sowohl in der herzchirurgischen Klinik als auch in der Rehabilitationsklinik unter kontinuierlicher Überwachung stand, erfolgt nun das Management des VAD in weitgehender Eigenständigkeit. Aus diesen Gründen sollte ein klar definiertes Entlassungssetting vorliegen. Das Potenzial zur Erfüllung der Selbstversorgungsziele sollte bei der Entlassung nach Hause vorliegen [171-173].

Entlassungskriterien Die Entlassung eines VAD-Patienten ist komplex und zeitaufwendig. Es empfiehlt sich, evtl. schon im implantierenden Zentrum eine Entlassungs-Checkliste (Tab. 15) anzulegen und diese während des Aufenthalts in der Akutklinik und nachfolgend in der Rehabilitation abzuarbeiten [11]. Auch hier sollte in Kooperation zwischen der implantierenden Klinik und der Rehabilitationseinrichtung die Zuständigkeit für die einzelnen Schritte geklärt sein.

Es ist darauf zu achten, dass eine für die Betroffenen und ihre Familien tragfähige und verantwortbare Versorgungsform organisiert wird. Vor der Entlassung sollte der Hausarzt des Patienten kontaktiert und eingewiesen werden. Des Weiteren sind die Erforderlichkeit von Hilfsmitteln nach dem Hilfsmittelverzeichnis sowie die Möglichkeit einer Pflegeeinstufung nach dem SGB XI zu prüfen. Gegebenenfalls sind entsprechende Verordnungen bzw. Anträge vorzubereiten. Im Einzelfall kann dies auch die Einleitung einer stationären Pflege in entsprechend eingewiesenen Pflegeeinrichtungen bedeuten.

\section{Mobilität des VAD-Patienten}

Aufgrund des guten Allgemeinzustandes und der Miniaturisierung der Systeme hat der VAD-Patient heutzutage die Möglichkeit, sich im Alltag weitgehend normal
Tab. 15 Entlassungs-Checkliste für VAD-Patienten

Zustand
Ordnungsgemäße Funktion des Systems
Suffiziente medikamentöse Therapie
Stabiler klinischer und funktioneller Status
Operationswunden im Wesentlichen verheilt
Stabile Flüssigkeitsbilanz
Keine Driveline-Infektion
Patient körperlich in der Lage, die Selbstversorgung (über-
wiegend) zu leisten
Stabiler psychologischer Status
Kompetenz
Sichere Kenntnisse im Gerätemanagement
Sicher im Wechseln der Stromquelle
Sicher in der Interpretation von Alarmen
Kompetent bei der Sicherung der Driveline
Erkennen von Gefahren
Identifizieren möglicher Schädigungsquellen des VADs zu Hause
und außerhalb
Beurteilung des Driveline-Austritts mithilfe einer
Fotodokumentation
Sicher im Umgang mit nicht therapeutischen INR-Werten
Erkennen von Anzeichen einer Magen-Darm-Blutung
Organisatorisch
Einweisung der Angehörigen
Vorbereitung der Wohnung
Gewährleistung der dauerhaften Stromversorgung
Kontaktaufnahme
Benachrichtigung und Einweisung des Hausarztes
Benachrichtigung und Einweisung der kardiologischen Abteilung
des zuweisenden Krankenhauses
Benachrichtigung und Einweisung des VAD-Koordinators des im-
plantierenden Zentrums
Benachrichtigung und Einweisung des Pflegedienstes zwecks
Driveline-Versorgung
Formulare

zu bewegen. Dazu gehört auch in zunehmendem Maße die Nutzung von Fortbewegungsmitteln.

Führen eines Kraftfahrzeuges mit mechanischer Herzunterstützung Das Führen eines Fahrzeuges kann für einen Patienten mit einem VAD ein entscheidender Faktor für seine Lebensqualität sein [129]. Das Risiko durch komplikationsbedingte Unfälle muss im Vorfeld abgewogen werden. Laut den amerikanischen Leitlinien ist die Klärung der Fahrtüchtigkeit eine Entscheidung des jeweiligen Implantationszentrums und wird auch durch die lokale Gesetzgebung beeinflusst [174, 175]. Untersuchungen aus den USA zeigen, dass mehr als $80 \%$ der zu Hause lebenden VAD-Patienten tatsächlich in kleinerem Rahmen als Fahrzeugführer am Straßenverkehr teilnehmen [176, 177]. 
Unfälle oder bedrohliche Komplikationen wurden dabei nicht angegeben.

Abwägende Stellungnahmen aus verkehrsmedizinischer Sicht liegen in Deutschland nicht vor. In der Fahrerlaubnisverordnung des Bundesministeriums für Justiz und Verbraucherschutz sowie in den Leitlinien der Deutschen Gesellschaft für Kardiologie wird dieses Thema nicht erwähnt. Im Falle der Herzinsuffizienz NYHA III ohne VAD wird eine bedingte oder vollständige Fahrtauglichkeit angegeben [178]. Andere Quellen geben im Falle eines VAD ein generelles Fahrverbot an [179, 180]. Sinnvoll erscheint uns die Empfehlung der Canadian Cardiovascular Society, die für Patienten mit Assist devices bei gutem Allgemeinzustand von einer Fahrtüchtigkeit im nichtkommerziellen Bereich ausgeht [181].

Entwickelt der Patient eine Synkope bzw. einen Insult, könnte in Anlehnung an die BAST (Bundesanstalt für Straßenwesen)-Leitlinien [182] für ICD-Patienten eine Fahruntüchtigkeit für einen festgelegten Zeitraum (z. B. 3-6 Monate nach dem Ereignis) festgelegt werden.

Das heißt zusammengefasst, dass die Fahrtüchtigkeit mit einem mechanischen Unterstützungssystem bei fehlenden wissenschaftlichen Daten eine individuelle Entscheidung nicht zuletzt auch des Fahrzeugführers - sein muss. Die Rehabilitation kann hier nur eine beratende Funktion für Betroffene und deren Angehörige übernehmen, vor allem dann, wenn Komplikationen der VAD-Therapie (Insult, kognitive Störungen, symptomatische Herzrhythmusstörungen) zu einer Einschränkung der Fahreignung führen.

\section{Benutzung öffentlicher Verkehrsmittel}

Die Benutzung von Bahn oder anderen öffentlichen Verkehrsmitteln ist im Alltag möglich. Die in den Fernzügen vorhandenen Stromanschlüsse bieten sogar die Möglichkeit, die Akkumulatoren während der Fahrt aufzuladen.

Schwierig wären nur akute gesundheitliche Problemen auf der freien Strecke, wenn dadurch ein unplanmäßiger Halt erforderlich wird oder wenn es zu Verspätungen und/ oder Stromausfällen kommen sollte.

\section{Reisen mit dem VAD}

Im Verlauf einer Rehabilitation werden sich seitens des Patienten möglicherweise Fragen zur Planung von Reiseaktivitäten ergeben. Das Team der betreuenden Rehabilitationsklinik hat diesbezüglich ggf. eine beratende Funktion zu übernehmen.

Fernreisen sollten aufgrund des zu erwartenden Aufwands und unter Berücksichtigung von möglichen Komplikationen im Reiseverlauf gut durchdacht und abgewogen werden. Die Vorbereitung einer Fernreise umfasst das Bereitstellen der Telefonnummern und Adressen aller VAD-Zentren entlang der Reiseroute. Weiterhin sollten medizinische Unterlagen wie ICD- oder Herzschrittmacherausweis und eine aktuelle Medikamentenliste mitgeführt werden. Des Weiteren ist auch an eine Mitnahme des INR-Messgerätes im Rahmen des Selbstmanagements zu denken. Bei Auslandsreisen muss unbedingt abgeklärt werden, ob landestypische Steckdosenadapter notwendig sind und ob die landesübliche Stromzufuhr mit der Ladestation der VADAkkus kompatibel ist.

Eine einmonatige Vorbereitungszeit für längere Reisen hat sich als sinnvoll erwiesen.

Reisen mit dem Flugzeug Bei Flugreisen ist es erforderlich, dass in der Vorbereitung die Fluggesellschaft mit einem speziellen Anschreiben (Abb. 10) informiert wird und nachfolgend ihr Einverständnis zur Mitnahme eines VAD-Patienten gibt. Das gemäß der Federal Aviation Administration (FAA) erstellte Schreiben erklärt, dass das VAD-System mit allen Sicherheitsanforderungen kompatibel ist und die Luftfahrtelektronik nicht stört. Letztendlich aber obliegt die Entscheidung über die Mitnahme der Patienten der Fluglinie. Ansonsten ist damit zu rechnen, dass bei einem durch den VAD-Träger verursachten Zwischenfall, der z. B. zu einer Zwischenlandung führen muss, erhebliche Regressforderungen auf den VAD-Träger zukommen könnten.

Die komplette VAD-Ausrüstung muss als Handgepäck an Bord gebracht werden. Es ist sinnvoll, das Sicherheitspersonal entsprechend zu informieren, um so den Check-in und das Boarding zu erleichtern. Zudem können Absprachen mit der Fluggesellschaft über eine erweiterte Betreuung und Begleitung während der Flugreise getroffen werden.

Die VAD-Träger müssen sich immer auf unerwartete Verzögerungen einstellen, wie z. B. schlechtes Wetter mit Änderung des Zielflughafen oder verzögerte bzw. abgesagte Anschlussflüge [183]. Man kann nicht davon ausgehen, dass während eines Fluges die Akkus geladen werden können [184]. Nach Herstellerangaben sollen sich der Kabinendruck und entsprechend auch Druckschwankungen nicht auf die Geräte auswirken. In diesem Zusammenhang sei auf den unter Flugbedingungen schwankenden Volumenstatus der Patienten hingewiesen. Es sollte detailliert auf eine gezielte Volumenzufuhr in Abhängigkeit von der Umgebungstemperatur geachtet werden [185]. Auf den Genuss von Alkohol sollte bei VAD-Trägern verzichtet werden.

\section{Reisen mit dem Schiff}

Im Falle von absolut stabilen Verläufen besteht bei entsprechendem Wunsch des Patienten nach gründlicher Abwägung durch die medizinischen Dienste der Reedereien die Möglichkeit, an einer Schiffsreise teilzunehmen [185]. In der Regel muss vor Antritt der Reise ein medizinischer 
Abb. 10 Sicherheitsbegleitschreiben für Flugreisen mit einem VAD-System [185]

\section{Notification Letter Template for Air Travel}

\section{DATE}

NAME

ADDRESS 1

CITY, STATE ZIP

To Whom It May Concern:

This letter is to inform you that one of your passengers, whose name and information is given below, has an implanted life sustaining device and requires special consideration in navigating the travel process.

\section{Patient Name \& DOB \\ Name of Companion}

The passenger has a Ventricular Assist Device (VAD), which is a mechanical heart pump. It is surgically placed inside the body and works alongside the heart to provide blood flow to the body in people with severe heart failure. A cable from the pump exits the body and is connected to a controller and a pair of batteries which provide control and power to the implanted pump.

I am requesting your assistance in helping this passenger through the security screening and boarding process. The passenger must avoid the electronic airport security equipment as this may interfere with the VAD. The passenger can be hand screened with special care given to the driveline cable exit site. Additional emergency back-up equipment will be required to be brought on board the aircraft with the passenger and be kept in his/her possession throughout the flight due to safety and emergency considerations.

The ventricular assist device is compliant with all related FAA safety requirements and will not interfere with aviation electronics, per Section 21, Category M of the RTCA document number RTCA/DO-160F, as specified in "Use of Portable Electronic Devices Aboard Aircraft" AC number 91.21-1B, Section 8A.

Thank you in advance for your assistance in helping this passenger through the flight check-in process. If you have any questions about the ventricular assist device, please do not hesitate to contact me.

Sincerely,

NAME

TITLE

HOSPITAL CONTACT INFORMATION
Fragebogen ausgefüllt werden, auf dem alle Angaben auch zum VAD enthalten sein sollten. Die Transportbewilligung wird durch die Reederei ausgestellt. Einige Reedereien lassen sich im Einzelfall nur auf die Mitnahme eines VADPatienten ein, wenn von diesem eine Haftungsentbindung unterschrieben wird. Generell sollte dem Reiseteilnehmer klar sein, dass im Falle einer Komplikation eine spezifische Behandlung an Bord nicht gewährleistet werden kann.
Wichtig bei Schiffsreisen ist die Sicherstellung der Stromversorgung an Bord. Eventuelle Kontaminationen des VAD-Systems mit Wasser (z. B. starke Gischt) sollten unbedingt vermieden werden. Im Falle eines Notfalls bzw. einer Havarie insbesondere mit Wassereinbruch sind im Vorfeld mögliche Maßnahmen zu planen, um das VADSystem zu schützen. 


\subsection{Standards für den Umgang mit Notfallsituationen} in der Rehabilitation von VAD-Patienten

Elementare Voraussetzung für einen sicheren Umgang mit Notfallsituationen von Patienten mit Kunstherzsystemen ist das Vorhandensein einer profunden qualitativen und quantitativen Expertise in dieser hochspezialisierten Versorgung. Diese Expertise kann nur dann bestehen, wenn die Behandlung von Patienten mit VAD einen Behandlungsschwerpunkt darstellt. Deshalb sollte die Rehabilitation von mindestens 25 VAD-Patienten pro Jahr angestrebt werden.

Es muss eine klar definierte Notfallversorgungskette etabliert sein unter Benennung einer rasch erreichbaren intensivmedizinisch kompetenten Akutabteilung mit überbrückendem Behandlungspotenzial sowie die Verlegungsplanung vom Rehabilitationszentrum in dieses Akuthaus und weiter von diesem Akuthaus in die Kardiochirurgie mit klar definierten Verlegungsmitteln und Verlegungszeiten. Diese Notfallkette sollte mit allen Ansprechpartnern (VADKoordinatoren, Intensivabteilung, VAD-Implantationszentrum, RTW, Hubschraubertransport, Hersteller-Hotline) mit allen Telefonnummern für eine 24-Stunden-Erreichbarkeit als Aushang an zentraler Stelle vorgehalten werden.

Die Kompetenz für den medizinischen Umgang mit kardiovaskulären Notfällen und Systemalarmen bei Patienten mit Kunstherzsystemen muss als Handlungsstandard im Rahmen des jeweiligen örtlichen Qualitätsmanagements festgelegt sein. Diese Kenntnisse sind durch Herstellereinweisungen für alle medizinisch betreuten Systeme (ggf. Wiederholungen/Aktualisierungen) sowie ärztlich autorisierte Schulungen regelmäßig dem Behandlungsteam bestehend aus Ärzten, Pflegepersonal sowie Sport- und Physiotherapeuten zu vermitteln und in regelmäßigen Wiederholungsschulungen $\mathrm{zu}$ sichern und zu vertiefen. Jedes Mitglied des Therapieteams muss dokumentiert zumindest $2 \times / \mathrm{Jahr}$ an einer entsprechenden VAD-Notfallschulung teilnehmen. Die Notfallschulungen sollen personell und logistisch (Schulungsmaterial, Notfallanleitungen) durch die Hersteller unterstützt werden. Hier sollte auch die Möglichkeit des E-Learnings genutzt werden. Die in Form von übersichtlichen Bildtafeln von den Systemherstellern zum Umgang mit den Systemkomponenten und zur Interpretation von Alarmsignalen zur Verfügung gestellten Anleitungen müssen für alle betreuten Kunstherzsysteme jederzeit problemlos einsehbar (z. B. Notfallordner Kunstherzsysteme) auf aktuellem Stand bereitgehalten werden.

Zum Notfallmanagement durch die Rehabilitationsklinik gehört auch Nacharbeit in der Schulung von Patienten und Angehörigen hinsichtlich des Verhaltens bei Systemalarmen und Herz-Kreislauf-Störungen. Dies beinhaltet die sichere Bereitstellung von kompakten Notfallinformationen für den Patienten, seine Angehörigen und dritte Ersthelfer zu Hause und unterwegs inklusive Notfall-Telefonnummern für den sofortigen Kontakt zu medizinisch kompetenten Ansprechpartnern bei jeder Tages- und Nachtzeit. In der Bereitstellung solcher Informationen kommt dem implantierenden VAD-Zentrum und den System-Herstellern allerdings eine primäre Rolle zu. Wünschenswert wäre die Vereinbarung von Standards für die Versorgung von VAD-Patienten durch die implantierenden Zentren für Kunstherzen, die über die operative Leistung hinaus die gesamte Nachsorge (VADAmbulanzen, 24-Stunden-Bereitschaft, Anforderungen an die Rehabilitation, Home-Care, Antikoagulationsmanagement, Driveline-Versorgung) möglichst optimal regeln.

Standards für Notfallsituationen von VAD-Patienten im Speziellen Die vorliegenden Handlungsstandards beruhen ganz wesentlich auf wissenschaftlicher Grundlage der Veröffentlichungen von Vierecke et al. sowie Slaughter et al. $[28,60]$.

Die möglichen Notfallsituationen können nach der Art des Notfalls strukturiert werden:

1. Primäres Energieproblem

2. Primäres Hardwareproblem (Controller, Pumpe, Driveline)

3. Primäres akutes kardiovaskuläres Problem (maligne Herzrhythmusstörung, Volumenmangel, Rechtsherzversagen, Tamponade, Schlaganfall, u. a.)

4. Primäres Problem durch maligne Blutung

Grundsätzlich sind neben den Besonderheiten durch das Vorhandensein eines VAD in hämodynamischen Notfallsituationen oder gar Reanimationssituationen die entsprechenden Leitlinien der medikamentösen Primärversorgung (Regulation der kardialen Vor- und Nachlast sowie eines wirksamen zentralen Blutdrucks, Terminierung maligner Arrhythmien) sowie die Leitlinien der kardialen Reanimation zu beachten.

Zunächst wird auch bei Patienten mit VAD initial zwischen Reanimationsnotfall (Bewusstlosigkeit/keine regelmäßige Spontanatmung)und Nicht-Reanimationsnotfall unterschieden. Im Reanimationsnotfall muss neben der prinzipiell sofort einzuleitenden Basisreanimation (Herzdruckmassage 100/min, Beatmung im Verhältnis 30:2) möglichst zeitgleich erfasst werden, ob ein rasch zu behebendes technisches Problem des Kunstherzsystems ursächlich ist.

Eine schnelle Orientierung zur Frage ,Arbeitet das Kunstherzsystem?" gibt bei einem Continuous-Flow-System die Auskultation über der Pumpe (,Summen“), im Notfall auch das Auflegen der Hand an dieser Stelle (,Vibrieren“). Für alle Kunstherzsysteme gilt, dass im Notfall unmittelbar die Stromversorgung (Akkus/Netz) und die Konnektion vom Antrieb zum Patienten (Driveline-Kabel) überprüft und bei Störung sofort hergestellt werden muss. Hinweise zur 
Dringlichkeit des Alarms geben dabei Alarmtöne und farbliche Alarmsignale am Controller bzw. auch deren Fehlen. Technische Sofortmaßnahmen sind demnach das Überprüfen der Steckverbindungen und deren Herstellung, falls unterbrochen, das Wechseln von Akkus, bzw. der Anschluss an den Netzstrom oder Notstrom (eine Energiequelle muss dabei immer am Controller sein) und der Controllerwechsel.

Im Falle eines Pumpenstopps besteht grundsätzlich die Gefahr einer Gerinnselbildung in der Pumpe. Die hängt von dem Antikoagulationsstatus und der Länge des Pumpenstopps ab. Bei kontinuierlich pumpenden Herzunterstützungssystemen, die keine integrierten Klappen benötigen, kommt es bei Stillstand der Pumpe zu einem retrograden Blutfluss aus der Aorta über die Pumpe in den linken Ventrikel, der unter normalen Blutdruckbedingungen z. B. bei einer HeartMate II $^{\circledR}$ etwa 1-2 1/min betragen kann. Nach Erfahrungen des Deutschen Herzzentrums Berlin kann eine Rotationspumpe nach bis zu 3-minütigem Stillstand problemlos wieder in Betrieb genommen werden. Längere Zeiten werden kontrovers diskutiert. Die ständige Verfügbarkeit eines Ersatz-Controllers ist bei ContinuousFlow-VADs die Regel. Ein EKG kann bei diesen Systemen anders als beim totalen Kunstherz (TAH) jederzeit abgeleitet werden. Die Beurteilung der Kammerendstrecke kann schwierig sein und erfordert den Vergleich mit Vor-EKGs. Der Herzrhythmus ist im Allgemeinen uneingeschränkt beurteilbar. Eine elektrische Kardioversion bzw. eine Defibrillation ist ohne Dekonnektierung des Controllers im laufenden Betrieb möglich.

Gegen die Herzdruckmassage bestehen seitens der Systemhersteller Bedenken. Sie wird jedoch bei Kreislaufstillstand, wenn das System nicht wieder in Gang gesetzt werden kann, von kardiochirurgischer Seite empfohlen [60].

Genauso wichtig wie ein kompetentes Management einer Notfallsituation mit einem VAD sollte ein proaktiver Ansatz zum frühzeitigen Erkennen und möglichst Vorbeugen einer Notfallsituation sein. Dazu müssen wesentliche medizinische Ausgangscharakteristika des jeweiligen Patienten gut erfasst werden (z. B. RV-Funktion bei LVAD, pulmonale Widerstände, Einstellung des VAD auf full support oder partial support, Einlagerung von freiem Wasser als Pleuraerguss, Perikarderguss, Aszites in der Vorgeschichte, Rhythmuslabilität, Vorhandensein eines ICD, Nierenfunktion, Diuretikabedarf und Risiken durch Elektrolytentgleisung, Systemalarme in der Vorgeschichte durch z. B. Ansaugphänomene oder Kanülenabknickung). Es muss ein Patientenprotokoll geführt werden mit Körpergewicht $1 \times$ tgl., Körpertemperatur $1-2 \times$ tgl., INR z. Zt. je nach Transplantationszentrum tgl. bis alle 2-3 Tage, Phenprocoumon-/Warfarin-Dosis tgl., Pumpflow, Power, Pulsatilitätsindex, Pumpspeed jeweils 1- bis mehrmals täglich. Diese Parameter sind kein Ersatz für die klinische Beurteilung des Patienten, können aber wertvolle Warnhin- weise auf drohende Komplikationen liefern. So können eine steigende Energieaufnahme und eine abnehmender Flow bei Kunstherzsystem mit kontinuierlichem Fluss Hinweis auf eine Thrombose in der Pumpe sein, eine sinkende Energieaufnahme mit sinkendem Fluss ein Hinweis auf Thrombose im Bereich des Inflow- oder Outflow-Trakts. Auffälligkeiten im Protokoll sollten zeitnah mit dem Transplantationszentrum besprochen werden und eine Kontrolle der Flüssigkeitsbilanz, des EKG und der LDH (Pumpenthrombose bei Rotationspumpen geht häufig mit LDH-Anstieg einher) veranlassen oder eine vorgezogene Kontrolle im VADAkutzentrum. Bei Rotationspumpen ist eine klassische auskultatorische oder palpatorische Blutdruckmessung nicht möglich. Mit oszillatorischen Blutdruckmessgeräten kann bei einem adäquaten Pulsatilitätsindex ein Blutdruckintervall bzw. ein mittlerer arterieller Blutdruck noch abgeschätzt werden. Der mittlere arterielle Blutdruck (MAD) sollte bei $70-80 \mathrm{mmHg}$ liegen. In dieser Größenordnung sind die blutdruckabhängigen Risiken von ischämischen oder hämorrhagischen zerebralen Insulten am geringsten. Auch sind alle VAD abhängig von Vor- und Nachlast. Volumenmangel kann bei Rotationssystemen zu Ansaugphänomen der Inflow-Kanüle führen, erhöhte Nachlast zu FlowMinderung. Die Vorgaben für ein „bridging“ des INR bei Unterschreitung (UFH, NMH, Fondaprinux, Argatroban bei HIT II) bzw. Gabe einer Einheit PPSB bei starker Überschreitung müssen durch das implantierende Zentrum klar definiert sein.

Im Folgenden werden einige Besonderheiten zu Notfallsituationen bei pulsatilen VAD-Systemen mit extrakorporalen Pumpenköpfen und beim Kunstherz (Total Artificial Heart - TAH) genannt, die im Vergleich zu den VAD mit kontinuierlichem Fluss eine vergleichsweise kleine Patientengruppe betreffen und für die auch eine besondere Expertise und ein besonderer Versorgungsstandard erforderlich ist.

Ein Pumpenstillstand ist durch das fehlende laute Pumpengeräusch leicht festzustellen. Fehlt trotz Pumpengeräusch beim parakorporalen System (extern liegende Pumpenköpfe) offensichtlich die Funktion der Blutpumpen, sollte als erstes die Konnektion zwischen Antrieb und Pumpe sowie Pumpe und Patient überprüft werden. Ein Leck an der pneumatischen Druckleitung ist, umgehend abgedichtet, keine schwerwiegende Komplikation. Sollte ein Riss an einer blutführenden Leitung auftreten, muss dieses Leck sofort abgedichtet werden. Sonst besteht die Gefahr einer Luftembolie und/oder eines erheblichen Blutverlusts. Bei großem nicht abdichtbarem Leck muss die entsprechende Pumpe sofort gestoppt werden, indem die Antriebsleitung dekonnektiert wird und die $\mathrm{Zu}-$ und Abfluss-Kanülen abgeklemmt werden. Bei biventrikulärem VAD sollte im Falle eines nicht wiederherstellbaren Ausfalls der linken Pumpe zur Vermeidung eines Lungenödems 
auch die rechte gestoppt werden. Eine Ruptur der Membran zwischen Blut- und Luftkammer bei einem System mit extrakorporalen Pumpenköpfen äußert sich in einer Kissenbildung zwischen den einzelnen Teilmembranen. Der Pumpenkopf muss umgehend ausgetauscht werden. Ein unmittelbares Risiko einer Embolie besteht meist nicht, da die Pumpenmembran mehrschichtig aufgebaut ist und fast immer nur eine Teilmembran einreißt. Ein bei weiterlaufendem Antrieb perakut aufgetretenes Pumpversagen durch intrakorporalen Defekt an einem Total Artificial Heart ist im Allgemeinen nicht mehr therapierbar. Ein Antriebsausfall bei einem TAH entspricht einem Kreislaufstillstand und muss sofort durch Wechseln auf den Ersatzantrieb behoben werden. Die Betreuung eines TAH-Patienten hat eine 24-Stunden-Überwachung mit sofortiger Bereitschaft zum Wechseln des Antriebs durch eine geschulte Person zur Voraussetzung. Als Alternative zur Anwesenheit im Raum kann eine Pulsoxymetrie-Überwachung und, wenn auch nicht standardisiert, eine akustische Funküberwachung erwogen werden, wobei für die genannten technischen Hilfsmittel auch eine Person erforderlich ist, die die entsprechenden Signale ununterbrochen registriert.

Für alle Kunstherzsysteme gilt, dass im Notfall unmittelbar die Stromversorgung (Akku/Netz) und die Konnektion vom Antrieb zum Patienten (Driveline, pneumatische Druckleitung) überprüft und bei Störung sofort wiederhergestellt werden muss.

Gefahrlose Inbetriebnahme nach Stillstand wird bei pulsatilen Systemen kontrovers diskutiert. Für das pulsatile Unterstützungssystem Excor $^{\circledR}$ der Fa. BerlinHeart ${ }^{\circledR}$ steht im Notfall eines Pumpenstopps auch eine Handpumpe zur Verfügung. Beim Handbetrieb ist auf eine komplette Füllung und Entleerung der Pumpe zu achten. Bei Ausfall eines Antriebs (links oder rechts) kann das Excor ${ }^{\mathbb{B}}$ der Fa. BerlinHeart $^{\circledR}$ unterbrechungsfrei auf einen Ersatzantrieb umschalten (Backup) und das PVAD der Fa. Thoratec ${ }^{\circledR}$ ein alternierendes Ansteuern des linken und rechten Pumpenkopfs durch den verbliebenen zur Überbrückung gewährleisten. Die ständige Verfügbarkeit eines ErsatzControllers/-Antriebs ist beim pulsatilen System Thoratec ${ }^{\circledR}$ und selbstverständlich beim TAH die Regel.

\subsection{Ethische und palliativmedizinische Aspekte bei der Betreuung von VAD-Patienten unter Beachtung juristischer Vorgaben}

Die Entscheidung zur Implantation eines Unterstützungssystems stellt für alle Beteiligten eine große Herausforderung dar. Anders als andere medizinische Eingriffe, die typischerweise zu einer Wiederherstellung der körperlichen Funktion führen und nur in einem kleinen Prozentsatz aufgrund unabänderlicher Risiken zu Misserfolgen führen, sind mit diesem Eingriff sehr divergierende
Hoffnungen und auch Leidenswege verbunden. So zeigte die REMATCH-Studie [130], dass viele Patienten gute Aussichten auf Verbesserung von Körperfunktionen und Verlängerung der Lebenszeit im Vergleich zu ausschließlich medikamentöser Therapie haben. Andererseits musste noch 2009 mit modernen Continous-Flow-Pumpen über einen Zeitraum von 2 Jahren in über $50 \%$ mit sogenannten schwerwiegenden unerwünschten Ereignissen gerechnet werden, u. a. $10 \%$ Re-Operation, $11 \%$ Schlaganfall, $33 \%$ Tod [64]. Diese Quote konnte in den folgenden Jahren in der HeartMate-II-Studie auf $41 \%$ gesenkt werden [186]. Da die Ergebnisse im Einzelfall ungewiss sind, ist mit erheblichen Belastungen nicht zuletzt auch für Pflegende und Familienangehörige zu rechnen [129, 187].

Die Mehrheit der Implantationen ist elektiv. Der Patient hat Zeit und Gelegenheit, für sich persönlich und unter Einbeziehung von Lebenspartnern hinsichtlich der Akzeptanz des VAD eine Entscheidung zu fällen, die seinen individuellen Wertvorstellungen entspricht [188]. Hier wird in der Literatur allerdings eine oft unzureichende Aufklärung mit ungenügender Transparenz einerseits und mangelhaftem Verständnis patientenseitig beklagt, zumal die ursprüngliche Indikationsgruppe „brigde to transplant“ und die ohnehin seltene „bridge to recovery“ zahlenmäßig immer kleiner werden in Relation zu ,destination therapy“ und der in letzter Zeit häufiger genutzten „brigde to decision“ [189]. Mit der verbesserten notfall- und intensivmedizinischen Versorgung mehren sich in kardiologischen Zentren jedoch auch die Fälle, in denen Patienten zum fraglichen Zeitpunkt ihre Entscheidung nicht kommunizieren können, sodass „Ersatzentscheider“ (Familienangehörige, durch Patientenverfügung oder behördlich bestellte Personen) befragt werden, die allerdings mindestens in 1/3 der Fälle anders entscheiden, als es der Betroffene getan hätte [190, 191].

Dieser Umstand sowie die unausweichliche Quote von Komplikationen und das Auftreten anderer Organfunktionsbeeinträchtigungen führen dazu, dass das medizinische Team zunehmend häufiger mit dem Thema „Lebensende am System“ konfrontiert wird [192, 193]. Grundsätzlich entsteht dann eine Situation, die vergleichbar ist mit der Entwicklung anderer unheilbarer Krankheiten und welcher mit palliativmedizinischen Standards begegnet werden sollte. Dies ist bisher in einem auf Rehabilitation ausgerichteten Umfeld durchaus ungewöhnlich. Dennoch ist nicht auszuschließen, dass in Absprache mit Implantationszentren zukünftig auch andere Institutionen als das Zentrum selbst sich dieser Aufgabe stellen müssen.

Implantationszentren in den USA haben seit längerem palliativmedizinische Dienste, die häufig bereits bei der Entscheidung zum LVAD beratend mitwirken und mit den Betroffenen eine strukturierte Vorsorgeplanung erarbeiten, die unteren anderem die Präferenzen des Betroffenen in verschiedenen Szenarien zum Ende des Lebens festhält, was 
wiederum zum späteren Zeitpunkt eine erhebliche Entlastung für das medizinische Team und Angehörige bedeutet [194196]. In Deutschland ist die Palliativmedizin lange Zeit als Synonym für eine Sparte gesehen worden, die sich der Unterstützung von Patienten mit unheilbaren malignen Tumoren widmet, weshalb Kardiologen bisher mit den Prinzipien der Palliativmedizin oft wenig vertraut sind. Diese hat nach WHODefinition zum Ziel eine Verbesserung der Lebensqualität von Patienten und deren Familien, die sich Problemen ausgesetzt sehen, die mit einer lebensbedrohlichen Erkrankung einhergehen. Sie erreicht dies durch Linderung von Leiden, durch frühzeitiges Erkennen, einwandfreies Assessment und Behandlung von Schmerzen und anderen Problemen physischer, psychosozialer und spiritueller Art. Die Deutsche Gesellschaft für Palliativmedizin hat durch ihre Arbeitsgruppe „Nicht-Tumorpatienten“ ein sehr nützliches Arbeitspapier zur stadiengerechten Palliativversorgung bei Herzinsuffizienz herausgegeben, desgleichen auch die ESC in 2009 [197, 198].

$\mathrm{Da}$ die fortgeschrittene Herzinsuffizienz häufig längere Phasen relativer Stabilität zwischen Exazerbationen aufweist, ist der palliativtherapeutische Ansatz hier deutlich von Sterbebegleitung und Hospiz zu unterscheiden. Ein wesentlicher Unterschied zur Palliativtherapie bei Krebserkrankungen ist dabei die unterschiedliche Handhabung von symptomlindernden und lebensverlängernden Therapien: Während bei Krebserkrankungen lebensverlängernde Chemotherapien eingestellt werden, da ihre Auswirkungen der Symptomlinderung entgegenstehen, besteht in der Therapie des Herzversagens überwiegend Konkordanz, indem therapeutisch lebensverlängernde Therapien gleichzeitig zur Symptomlinderung beitragen [193]. Im Rahmen der palliativen Therapie kann auch die Deaktivierung des VAD erforderlich werden. In der öffentlichen Diskussion gehen US-Medizinethiker mehrheitlich davon aus, dass die Abschaltung ethisch erlaubt ist, wenn Patient oder Ersatzentscheider es fordern, da jeder Patient das Recht hat, eine lebensverlängernde Behandlung $\mathrm{zu}$ akzeptieren oder $\mathrm{zu}$ verweigern $[199,200]$. Die deutsche „Versorgungsleitlinie chronische Herzinsuffizienz" befasst sich zwar mit der Möglichkeit der ICD-Inaktivierung, erwähnt jedoch noch nicht die Unterstützungssysteme in entsprechender Situation [169]. Allgemein formulierte die Bundesärztekammer 2011, dass das Sterben durch die Beendigung einer begonnenen Behandlung ermöglicht werden darf, wenn dies dem Willen des Patienten entspricht [201]. Typische Szenarien sind zum Beispiel schwerer Schlaganfall, Multiorganversagen, fortgeschrittene Krebserkrankung oder Demenz. Eine Untersuchung der Mayo-Klinik fand in 6 Jahren bei 26 Verstorbenen eine Deaktivierung auf Wunsch bei $54 \%$. Nur 2 Patienten waren selbst entscheidungsfähig, für die Mehrheit mussten Angehörige entscheiden, da Patientenverfügungen zu unpräzise oder nicht auffindbar waren. Die Deaktivierung wird als „Erlaubnis zu sterben“ und nicht als
„Tötung“ verstanden. Dennoch gibt es hierzu unterschiedliche Einstellungen, und kein Mitglied im Arzt- oder Pflegeteam sollte genötigt werden, gegen sein Gewissen an einer Deaktivierung mitzuwirken [202, 203] Diese sollte nach Entscheidung im interdisziplinären Team in der Regel durch ein Mitglied des VAD-Teams in palliativstationärem Umfeld unter angemessener Sedierung erfolgen, wobei die mögliche Anwesenheit von Partnern oder Angehörigen abzustimmen ist. Zumeist verstarben Patienten innerhalb von $20 \mathrm{~min}$, in Einzelfällen innerhalb von 24 h [192, 203, 204].

\section{Appendix}

Für ein kritisches Lektorat bedanken wir uns bei Prof. Dr. Gernot Klein (Bernried am Starnberger See).

\subsection{Autorenerklärung}

Die Autoren erklären, dass sie keine finanzielle Verbindung mit einer Firma haben, deren Produkt in diesen Rehabilitationsstandards eine Rolle spielt (oder mit einer Firma, die ein Konkurrenzprodukt vertreibt).

\subsection{Weitere Mitwirkende}

R. Ballmann, Schüchtermann-Klinik, Bad Rothenfelde

T. Gampert, Klinik Roderbirken, Leichlingen

L. Matheis, Klinik Fallingbostel, Bad Fallingbostel

H. Montanus, Rehabilitationsklinik Elbe-Saale, Barby

\subsection{Abkürzungsverzeichnis}

$\begin{array}{lll}\text { 6-MWD } & \text { 6-Minutes Walking } \\ & \text { Distance/6-Minuten-Gehstrecke } \\ \text { AK-VAD }= & \text { Arbeitskreis Ventricular Assist Device } \\ \text { ASA } & \text { Arbeitsgemeinschaft Selbstkontrolle der Anti- } \\ & \text { koagulation e. V. } \\ \text { ATL } & =\text { Aktivitäten des täglichen Lebens } \\ \text { ATS } & =\text { American Thoracic Society } \\ \text { BTR } & =\text { Bridge to recovery } \\ \text { BTT } & =\text { Bridge to transplant } \\ \text { BVAD } & =\text { Biventricular assist device } \\ \text { CCM } & =\text { Cardiac contractility modulation } \\ \text { CRT-D } & =\text { Cardiale Resynchronisationstherapie mit } \\ & =\text { Defibrillator } \\ \text { DGK } & \text { Deutsche Gesellschaft für Kardiologie } \\ \text { DGPR } & =\text { Deutsche Gesellschaft für Prävention und Re- } \\ & =\text { habilitation von Herz- Kreislauferkrankungen e. V. } \\ \text { DT } & =\text { Destination therapy } \\ \text { EGK } & \text { Elektrokardiogramm } \\ \text { ERI } & =\text { Elective replacement intervall } \\ \text { ESC } & =\text { European Society of Cardiology }\end{array}$




\begin{tabular}{|c|c|c|}
\hline 6-MWD & $=$ & $\begin{array}{l}\text { 6-Minutes Walking } \\
\text { Distance/6-Minuten-Gehstrecke }\end{array}$ \\
\hline FIM & $=$ & Functional Independence Measure \\
\hline $\mathrm{HF}$ & $=$ & Herzfrequenz \\
\hline HIT & $=$ & Heparininduzierte Thrombozytopenie \\
\hline HTX & $=$ & Herztransplantation \\
\hline $\mathrm{HZV}$ & $=$ & Herzzeitvolumen \\
\hline ICD & $=$ & Internal Cardioverter-Defibrillator \\
\hline ICF & $=$ & $\begin{array}{l}\text { International Classification of Functioning, } \\
\text { Disability and Health }\end{array}$ \\
\hline INR & $=$ & International Normalized Ratio \\
\hline ISHLT & $=$ & $\begin{array}{l}\text { The International Society for Heart \& Lung } \\
\text { Transplantation }\end{array}$ \\
\hline $\mathrm{KG}$ & $=$ & Krankengymnastik \\
\hline LVAD & $=$ & Left ventricular assist device \\
\hline LZ-EKG & $=$ & Langzeit-Elektrokardiogramm \\
\hline MRGN & $=$ & Multiresistente gramnegative Erreger \\
\hline MRSA & $=$ & Methicillin-resistente Staphylococcus aureus \\
\hline Newton $_{\max }$ & $=$ & Maximal erreichte Kraft in Newton \\
\hline $\mathrm{NMH}$ & $=$ & Niedermolekulares Heparin \\
\hline NOAK & $=$ & Neue orale Antikoagulanzien \\
\hline pAVK & $=$ & Periphere arterielle Verschlusskrankheit \\
\hline PPSB & & Prothrombinkonzentrat \\
\hline RPE & $=$ & Rate of perceived exertion, Borg-Wert \\
\hline RQ & $=$ & Respiratorischer Quotient \\
\hline RVAD & $=$ & Right ventricular assist device \\
\hline TENS & $=$ & Transkutane elektrische Nervenstimulation \\
\hline TET & $=$ & Transkutaner Energietransfer \\
\hline UFH & & Unfraktioniertes Heparin \\
\hline VAD & $=$ & Ventricular assist device \\
\hline VAT & $=$ & Ventilatorische anaerobe Schwelle \\
\hline $\begin{array}{l}\mathrm{VE} / \mathrm{CO}_{2}^{-} \\
\text {Slope }\end{array}$ & $=$ & $\begin{array}{l}\text { Steigerung der Beziehung zwischen Ventilation } \\
\text { und Kohlendioxidproduktion }\end{array}$ \\
\hline $\mathrm{VO}_{2 \text { peak }}$ & $=$ & Maximal erreichte Sauerstoffaufnahme \\
\hline $\mathrm{VO}_{2} \mathrm{R}$ & $=$ & Reserve der Sauerstoffaufnahme \\
\hline VRE & $=$ & Vancomycin-resistente Enterokokken \\
\hline Watt $_{\max }$ & $=$ & Maximal erreichte Wattzahl \\
\hline WHO & $=$ & World Health Organisation \\
\hline
\end{tabular}

Open Access Dieser Artikel unterliegt den Bedingungen der Creative Commons Attribution License. Dadurch sind die Nutzung, Verteilung und Reproduktion erlaubt, sofern der/die Originalautor/en und die Quelle angegeben sind.

\section{Literatur}

1. Statistisches Bundesamt Gesundheit. Todesursachen in Deutschland 2012. https://www.destatis.de/DE/Publikationen/Thematisch/ Gesundheit/Todesursachen/Todesursachen2120400127004. pdf?_blob=publicationFile. Zugegriffen: 15. Jan 2016

2. Statistisches Bundesamt Gesundheit. Diagnosedaten der Patienten und Patientinnen in Krankenhäusern (einschl. Sterbe- und Stundenfälle) 2011. https://www.destatis.de/DE/Publikationen/ Thematisch/Gesundheit/Krankenhaeuser/DiagnosedatenKranken haus2120621117004pdf?_blob=publicationFile. Zugegriffen: 15 . Jan 2016
3. Eurotransplant International Foundation Active heart waiting list (at year-end) in All ET, by year, by country, Report: 3071P All ET heart. http://statistics.eurotransplant.org/reportloader. php? report $=56893-6141-5853 \&$ format $=$ html\&download $=0, \quad \mathrm{Zu}-$ gegriffen: 15. Jan 2016

4. Eurotransplant International Foundation Heart donation, deceased donors in Germany, by year, by allocation phase, Report: 1142P Germany_heart. http://statistics.eurotransplant.org/reportloader. php?report $=55359-6010-5853 \&$ format $=$ html\&download $=0, \quad \mathrm{Zu}-$ gegriffen: 15. Jan 2016

5. Beyersdorf F (2009) Heart transplant and artificial heart systems. Dtsch Arztebl Int 106(28-29):469-70

6. German Society for Thoracic and Cardiovascular Surgery GSTCVS Annual Registry. Survey Period 2014. https://idwonline.de/de/attachmentdata43088.pdf. Zugegriffen: 13. Mai 2015

7. Kirklin J, Naftel D, Pagani F, Kormos R, Stevenson L, Blume E, Miller M, Timothy Baldwin J, Young J (2014) Sixth INTERMACS annual report: a 10,000-patient database. J Heart Lung Transplant 33(6):555-564

8. Franke I (2014) Kreislaufunterstützungssysteme: Auf dem Weg zum Dauereinsatz. Dtsch Arztebl Int 111(1-2):A-31-A-32

9. Workowski A, Willemsen D (2015) Befragung der kardiologischen Rehabilitationseinrichtungen in Deutschland. Nicht publizierte Statistik (für den AK-VAD). Bad Rothenfelde

10. Corrà U, Pistono $M$, Mezzani $A$, Gnemmi M, Tarro Genta $F$, Caruso R, Giannuzzi P (2011) Cardiovascular prevention and rehabilitation for patients with ventricular assist device from exercise therapy to long-term therapy. Part I: exercise therapy. Monaldi Arch Chest Dis 76(1):27-32

11. Pistono $\mathrm{M}$, Corrà U, Gnemmi M, Imparato A, Caruso R, Balestroni G, Tarro Genta F, Angelino E, Giannuzzi P (2011) Cardiovascular prevention and rehabilitation for patients with ventricular assist device from exercise therapy to long-term therapy. Part II: longterm therapy. Monaldi Arch Chest Dis 76(3):136-145

12. Vanhees L, Rauch B, Piepoli M, van Buuren F, Takken T, Borjesson M, Bjarnason-Wehrens B, Doherty P, Dugmore D, Halle M (2012) Importance of characteristics and modalities of physical activity and exercise in the management of cardiovascular health in individuals with cardiovascular disease (Part III). Eur J Prev Cardiol 19(6):1333-1356

13. Feldman D, Pamboukian S, Teuteberg J, Birks E, Lietz K, Moore S, Morgan J, Arabia F, Bauman M, Buchholz H, Deng M, Dickstein M, El-Banayosy A, Elliot T, Goldstein D, Grady K, Jones K, Hryniewicz K, John R, Kaan A, Kusne S, Loebe M, Massicotte M, Moazami N, Mohacsi P, Mooney M, Nelson T, Pagani F, Perry W, Potapov E, Eduardo Rame J, Russell S, Sorensen E, Sun B, Strueber M, Mangi A, Petty M, Rogers J (2013) The 2013 International Society for heart and lung transplantation guidelines for mechanical circulatory support: executive summary. J Heart Lung Transplant 32(2):157-187

14. AQUA GmbH: Weiterentwicklung des Leistungsbereiches Herztransplantation. Abschlussbericht, Stand: 15. März 2014. https:// www.sqg.de/sqg/upload/CONTENT/Themen/Leistungsbereiche/ HTX/Abschlussbericht_WE_LB_HTX.pdf. Zugegriffen: 19. Mai 2015

15. Pinkster I, Burgt B, Janssen D, Veenendaal E (2004) Successful test management. An Integral Approach. Springer, Dordrecht

16. Deutsche Rentenversicherung Bund Anforderungsprofil zur Durchführung der Medizinisch-beruflich orientierten Rehabilitation (MBOR) im Auftrag der Deutschen Rentenversicherung. Stand 2015. http://www.deutsche-rentenversicherung.de/cae/servlet/ contentblob/207024/publicationFile/50641/mbor_datei.pdf. Zugegriffen: 13. Juli 2015 
17. Gemeinsamer Bundesausschuss (BA) (2014) Richtlinie des Gemeinamen Bundesausschusses über Leistungen zur medizinischen Rehabilitation. vom 16. März 2004, zuletzt geändert am 17. April 2014. Bundesanzeiger BAnz AT 26. Juni 2014 B4

18. Collin C (2003) Measurement of disabillity and handicap. In: Greenwood R (Hrsg) Handbook of neurological rehabilitation. Psychology Press, Hove

19. Bundesarbeitsgemeinschaft für Rehabilitation (BAR) (2005) Rahmenempfehlungen zur ambulanten kardiologischen Rehabilitation. http://www.bar-frankfurt.de/fileadmin/dateiliste/ publikationen/empfehlungen/downloads/Rahmenempfehlung kardiologische_Rehabilitation.pdf. Zugegriffen: 7. Juli 2015

20. Bundesverband Deutscher Privatkrankenanstalten e. V. (2005) Rahmenkonzept zur stationären Rehabilitation bei kardiologischen Erkrankungen. http://www.bdpk.de/media/file/81. Rahmenkonzept_zur_stationaeren_Rehabilitation_bei_kardiologischen_Erkrankungen.pdf. Zugegriffen: 7. Juli $20 \overline{15}$

21. Deutsche Rentenversicherung Bund(2009) Rahmenkonzept zur medizinischen Rehabilitation in der gesetzlichen Rentenversicherung. 3. Auflage. http://www.deutsche-rentenversicherung. de/cae/servlet/contentblob/207036/publicationFile/2127/rahmenkonzept_medizinische_reha.pdf. Zugegriffen: 7. Juli 2015

22. Deutsche Rentenversicherung Bund Reha-Qualitätssicherung der Deutschen Rentenversicherung: Strukturqualität von Reha-Einrichtungen - Anforderungen der Deutschen Rentenversicherung, 2. überarbeitete und erweiterte Auflage, Juli 2014. http:// www.deutsche-rentenversicherung.de/Allgemein/de/Inhalt/3 Infos_fuer_Experten/01_sozialmedizin_forschung/downloads/ quali_strukturqualitaet/Broschuere_Strukturanforderungen. pdf?_blob=publicationFile\&v=9. Zugegriffen: 15. Jan 2016

23. Nolan JP, Soar J, Zideman DA, Biarent D, Bossaert LL, Deakin C, Koster RW, Wyllie J, Böttiger B (2010) European resuscitation council guidelines for resuscitation 2010 Section 1. Executive summary. Resuscitation 81(10):1219-1276

24. Pagani F, Miller L, Russell S, Aaronson K, John R, Boyle A, Conte J, Bogaev R, MacGillivray T, Naka Y, Mancini D, Massey H, Chen L, Klodell C, Aranda J, Moazami N, Ewald G, Farrar D, Frazier O (2009) Extended mechanical circulatory support with a continuous-flow rotary left ventricular assist device. J Am Coll Cardiol 54(4):312-321

25. Fang J (2009) Rise of the machines - left ventricular assist devices as permanent therapy for advanced heart failure. N Engl J Med 361(23):2282-2285

26. HeartWare ${ }^{\circledR}$ Inc (2009) Heart Ware - Ventrikuläres Unterstützungssystem. Gebrauchsanweisung

27. Slaughter M, Pagani F, McGee E, Birks E, Cotts W, Gregoric I, Howard Frazier O, Icenogle T, Najjar S, Boyce S, Acker M, John R, Hathaway D, Najarian K, Aaronson K (2013) HeartWare ventricular assist system for bridge to transplant: combined results of the bridge to transplant and continued access protocol trial. J Heart Lung Transplant 32(7):675-683

28. Slaughter M, Pagani F, Rogers J, Miller L, Sun B, Russell S, Starling R, Chen L, Boyle A, Chillcott S, Adamson R, Blood M, Camacho M, Idrissi K, Petty M, Sobieski M, Wright S, Myers T, Farrar D (2010) Clinical management of continuous-flow left ventricular assist devices in advanced heart failure. J Heart Lung Transplant 29(4):S1-S39

29. Strueber M, O'Driscoll G, Jansz P, Khaghani A, Levy W, Wieselthaler G (2011) Multicenter evaluation of an intrapericardial left ventricular assist system. J Am Coll Cardiol 57(12):1375-1382

30. Thoratec Corporation (2008) HeartMate II ${ }^{\circledR}$ LVAS: instructions for use. Thoratec Corporation, Pleasanton

31. HeartWare ${ }^{\circledR}$ Inc. http://www.heartware.com/sites/default/files/ fields/library/hvad-handshot-palm-high.jpg. Zugegriffen: 29. Juli 2015
32. Thoratec Corporation per Mailkontakt zugeschickte und zur Veröffentlichung freigegebene Bilddatei - Reprinted with the permission of Thoratec Corporation. Zugegriffen: 20. August 2015

33. HeartWare ${ }^{\circledR}$ Inc. per Mailkontakt zugeschickte und zur Veröffentlichung freigegebene Bilddateien. 10.8.2015

34. HeartWare ${ }^{\circledR}$ Inc. http://www.heartware.com/sites/default/files/ fields/library/monitor-with-controller-and-batteries-high.jpg. $\mathrm{Zu}-$ gegriffen: 29. Juli 2015

35. Hatano M, Kinugawa K, Shiga T, Kato N, Endo M, Hisagi M, Nishimura T, Yao A, Hirata Y, Kyo S, Ono M, Nagai R (2011) Less frequent opening of the aortic valve and a continuous flow pump are risk factors for postoperative onset of aortic insufficiency in patients with a left ventricular assist device. Circ J 75(5):1147-1155

36. Mezzani A, Pistono M, Corrà U, Giordano A, Gnemmi M, Imparato A, Centofanti P, Rinaldi M, Colombo S, Canal E, Giannuzzi P (2014) Systemic perfusion at peak incremental exercise in left ventricular assist device recipients: partitioning pump and native left ventricle relative contribution. IJC Heart Vessels 4:40-45

37. Myers T, Bolmers M, Gregoric I, Kar B, Frazier O (2009) Assessment of arterial blood pressure during support with an axial flow left ventricular assist device. J Heart Lung Transplant 28(5):423-427

38. Ammar K, Umland M, Kramer C, Sulemanjee N, Jan M, Khandheria B, Seward J, Paterick T (2012) The ABCs of left ventricular assist device echocardiography: a systematic approach. Eur Heart J Cardiovasc Imaging 13(11):885-899

39. Buck T, Breithardt O, Faber L, Fehske W, Flachskampf F, Franke A, Hagendorff A, Hoffmann R, Kruck I, Kücherer H, Menzel T, Pethig K, Tiemann K, Voigt J, Weidemann F, Nixdorff U (2009) Manual zur Indikation und Durchführung der Echokardiographie. Clin Res Cardiol Suppl 4(S1):3-51

40. Lang R, Bierig M, Devereux R, Flachskampf F, Foster E, Pellikka P, Picard M, Roman M, Seward J, Shanewise J, Solomon S, Spencer K, St John Sutton M, Stewart W (2005) Recommendations for chamber quantification: a report from the American Society of Echocardiography's Guidelines and Standards Committee and the Chamber Quantification Writing Group, developed in conjunction with the European Association of Echocardiography, a branch of the European Society of Cardiology. J Am Soc Echo 18(12):1440-1463

41. Quiñones MA, Otto CM, Stoddard M, Waggoner A, Zoghbi WA (2002) Recommendations for quantification of Doppler echocardiography. A report from the Doppler quantification task force of the nomenclature and standards committee of the American Society of Echocardiography. J Am Soc Echocardiogr 15(2):167-184

42. Kirkpatrick J, Wiegers S, Lang R (2010) Left ventricular assist devices and other devices for end-stage heart failure: utility of echocardiography. Curr Cardiol Rep 12(3):257-264

43. Rasalingam R, Johnson S, Bilhorn K, Huang P, Makan M, Moazami N, Pérez J (2011) Transthoracic echocardiographic assessment of continuous-flow left ventricular assist devices. J Am Soc Echo 24(2):135-148

44. Topilsky Y, Maltais S, Oh J, Atchison F, Perrault L, Carrier M, Park S (2011) Focused review on transthoracic echocardiographic assessment of patients with continuous axial left ventricular assist devices. Cardiol Res Pract 2011:1-11

45. Topilsky Y, Oh J, Atchison F, Shah D, Bichara V, Schirger J, Kushwaha S, Pereira N, Park S (2011) Echocardiographic findings in stable outpatients with properly functioning HeartMate II left ventricular assist devices. J Am Soc Echo 24(2):157-169

46. Stainback RF, Estep JD, Agler DA, Birks EJ, Bremer M, Hung J, Kirkpatrick JN, Rogers JG, Shah NR (2015) Echocardiography in the management of patients with left ventricular assist devices: recommendations from the American Society of Echocardiography. J Am Soc Echocardiogr 28(8):853-909 
47. Martina JR, Westerhof BE, van Goudoever J, Jonge N de, van Lieshout JJ, Lahpor JR, De Mol BAJM (2010) Noninvasive blood pressure measurement by the Nexfin monitor during reduced arterial pulsatility. A feasibility study. ASAIO J 56(3):221-227

48. Lanier G, Orlanes K, Hayashi Y, Murphy J, Flannery M, Te-Frey R, Uriel N, Yuzefpolskaya M, Mancini D, Naka Y, Takayama H, Jorde U, Demmer R, Colombo P (2013) Validity and reliability of a novel slow cuff-deflation system for noninvasive blood pressure monitoring in patients with continuous-flow left ventricular assist device. Circ Heart Fail 6(5):1005-1012

49. McMurray J, Adamopoulos S, Anker S, Auricchio A, Bohm M, Dickstein K, Falk V, Filippatos G, Fonseca C, Gomez-Sanchez M, Jaarsma T, Kober L, Lip G, Maggioni A, Parkhomenko A, Pieske B, Popescu B, Ronnevik P, Rutten F, Schwitter J, Seferovic P, Stepinska J, Trindade P, Voors A, Zannad F, Zeiher A, Bax J, Baumgartner H, Ceconi C, Dean V, Deaton C, Fagard R, FunckBrentano C, Hasdai D, Hoes A, Kirchhof P, Knuuti J, Kolh P, McDonagh T, Moulin C, Reiner Z, Sechtem U, Sirnes P, Tendera M, Torbicki A, Vahanian A, Windecker S, Bonet L, Avraamides P, Ben Lamin H, Brignole M, Coca A, Cowburn P, Dargie H, Elliott P, Flachskampf F, Guida G, Hardman S, Iung B, Merkely B, Mueller C, Nanas J, Nielsen O, Orn S, Parissis J, Ponikowski P (2012) ESC Guidelines for the diagnosis and treatment of acute and chronic heart failure 2012: the Task Force for the Diagnosis and Treatment of Acute and Chronic Heart Failure 2012 of the European Society of Cardiology. Developed in collaboration with the Heart Failure Association (HFA) of the ESC. Eur Heart J 33(14):1787-1847

50. Scheiderer R, Belde C, Schwab D, Haney C, Praz J (2013) Exercise guidelines for inpatients following ventricular assist device placement: a systematic review of the literature. Cardiopulm Phys Ther J 24(12):35-42

51. Trappe H, Löllgen H (2000) Leitlinien zur Ergometrie. Z Kardiol 89:821-837

52. Corrà U, Piepoli MF, Adamopoulos S, Agostoni P, Coats AJS, Conraads V, Lambrinou E, Pieske B, Piotrowicz E, Schmid J-P, Seferović PM, Anker SD, Filippatos G, Ponikowski PP (2014) Cardiopulmonary exercise testing in systolic heart failure in 2014: the evolving prognostic role: a position paper from the committee on exercise physiology and training of the heart failure association of the ESC. Eur J Heart Fail 16(9):929-941

53. Loyaga-Rendon RY, Plaisance EP, Arena R, Shah K (2015) Exercise physiology, testing, and training in patients supported by a left ventricular assist device. J Heart Lung Transplant 34(8):1005-1016

54. American Thoracic Society (ATS) (2002) ATS Statement: guidelines for the six-minute walk test. Am J Respir Crit Care Med 166(1):111-117

55. Podsiadlo D, Richardson S (1991) The timed „Up \& Go“. A test of basic functional mobility for frail elderly persons. J Am Geriatr Soc 39(2): $142-148$

56. Wonisch M, Hofmann P, Pokan R, Kraxner W, Hödl R, Maier R, Watzinger N, Smekal G, Klein W, Fruhwald F (2003) Spiroergometrie in der Kardiologie - Grundlagen der Physiologie und Terminologie. J Kardiol 10(9):383-390

57. Fess E (1982) The effect of Jamar dynamometer handle position and test protocol on normal grip strength. J Hand Surg Am 7:308-309

58. Marschall F, Gail S (2011) Standardisierung eines Testprotokolls zur Bestimmung der isometrischen Maximalkraft. Beweg Gesundh 27(06):226-230

59. Krabatsch T, Potapov E, Soltani S, Dandel M, Falk V, Knosalla C (2015) Ventrikuläre Langzeitunterstützung mit implantierbaren kontinuierlichen Flusspumpen. Auf dem Weg zum Goldstandard in der Therapie der terminalen Herzinsuffizienz. Herz 40(2):231-239
60. Vierecke J, Kaufmann F, Schweiger M, Stepanenko A, Dranishnikov N, Lehmkuhl H, Potapov E, Hetzer R, Krabatsch $\mathrm{T}$ (2011) Erste Maßnahmen und Verhalten bei Störungen eines Kunstherzsystems. Notfall Rettungsmed 14(8):635-646

61. Wilson SR, Givertz MM, Stewart GC, Mudge GH (2009) Ventricular assist devices the challenges of outpatient management. J Am Coll Cardiol 54(18):1647-1659

62. Garan A, Iyer V, Whang W, Mody K, Yuzefpolskaya M, Colombo P, Te-Frey R, Takayama H, Naka Y, Garan H, Jorde U, Uriel N (2014) Catheter ablation for ventricular tachyarrhythmias in patients supported by continuous-flow left ventricular assist devices. ASAIO J 60(3):311-316

63. Herweg B, Ilercil A, Kristof-Kuteyeva O, Rinde-Hoffman D, Caldeira C, Mangar C, Karlinosky R, Barold S (2012) Clinical observations and outcome of ventricular tachycardia ablation in patients with left ventricular assist devices. Pacing Clin Electrophysiol 35(11):1377-1383

64. Slaughter M, Rogers J, Milano C, Russell S, Conte J, Feldman D, Sun B, Tatooles A, Delgado R, Long J, Wozniak T, Ghumman W, Farrar D, Frazier O (2009) Advanced heart failure treated with continuous-flow left ventricular assist device. N Engl J Med 361(23):2241-2251

65. Oswald H, Klein G, Strüber M, Gardiwal A (2009) Implantable defibrillator with left ventricular assist device compatibility. Interact Cardiovasc Thorac Surg 8(5):579-580

66. Oswald H, Schultz-Wildelau C, Gardiwal A, Lüsebrink U, König T, Meyer A, Duncker D, Pichlmaier MA, Klein G, Strüber $M$ (2010) Implantable defibrillator therapy for ventricular tachyarrhythmia in left ventricular assist device patients. Eur J Heart Fail 12(6):593-599

67. Pecha S, Wilke I, Bernhardt A, Hakmi S, Yildirim Y, Steven D, Reichenspurner H, Willems S, Deuse T, Aydin A (2014) Clinical experience of combined HeartWare ventricular assist device and implantable cardioverter defibrillator therapy. J Cardiovasc Electrophysiol 25(10):1109-1114

68. Andersen M, Videbæk R, Boesgaard S, Sander K, Hansen P, Gustafsson F (2009) Incidence of ventricular arrhythmias in patients on long-term support with a continuous-flow assist device (HeartMate II). J Heart Lung Transplant 28(7):733-735

69. Refaat M, Tanaka T, Kormos R, Mcnamara D, Teuteberg J, Winowich S, London B, Simon M (2012) Survival benefit of implantable cardioverter-defibrillators in left ventricular assist device supported heart failure patients. J Cardiac Fail 18(2):140-145

70. Enriquez A, Calenda B, Miller M, Anyanwu A, Pinney S (2013) The role of implantable cardioverter-defibrillators in patients with continuous flow left ventricular assist devices. Circ Arrhythm Electrophysiol 6(4):668-674

71. Cantillon D, Tarakji K, Kumbhani D, Smedira N, Starling R, Wilkoff B (2010) Improved survival among ventricular assist device recipients with a concomitant implantable cardioverterdefibrillator. Heart Rhythm 7(4):466-471

72. Garan A, Yuzefpolskaya M, Colombo P, Morrow J, Te-Frey R, Dano D, Takayama H, Naka Y, Garan H, Jorde U, Uriel N (2013) Ventricular arrhythmias and implantable cardioverter-defibrillator therapy in patients with continuous-flow left ventricular assist devices. J Am Coll Cardiol 61(25):2542-2550

73. Pettit S, Petrie M, Connelly D, Japp A, Payne J, Haj-Yahia S, Gardner R (2012) Use of implantable cardioverter defibrillators in patients with left ventricular assist devices. Eur J Heart Fail 14(7):696-702

74. Muratsu J, Hara M, Mizote I, Asano Y, Sakata Y, Saito S, Matsumiya G, Sawa Y, Komuro I (2011) The impact of cardiac resynchronization therapy in an end-stage heart failure patient with a left ventricular assist device as a bridge to recovery. Int Heart J 52(4):246-247 
75. Ambardekar A, Lowery C, Allen L, Cannon A, Cleveland J, Lindenfeld J, Brieke A, Sauer W (2010) Effect of left ventricular assist device placement on preexisting implantable cardioverterdefibrillator leads. J Cardiac Fail 16(4):327-331

76. Moss A, Schuger C, Beck C, Brown M, Cannom D, Daubert J, Estes N, Greenberg H, Hall W, Huang D, Kautzner J, Klein H, McNitt S, Olshansky B, Shoda M, Wilber D, Zareba W (2012) Reduction in inappropriate therapy and mortality through ICD programming. N Engl J Med 367(24):2275-2283

77. Reibis RK, Kamke W, Langheim E, Völler H (2010) Rehabilitation von Patienten mit Herzschrittmachern und KardioverterDefibrillatoren: Empfehlungen zu Training, Physiotherapie und beruflicher Wiedereingliederung. Dtsch Med Wochenschr 135(15):759-764

78. Volkmann H (2004) Störbeeinflussung von implantierten Herzschrittmachern im medizinischen Bereich. Herzschr Elektrophys 15(1):65-72

79. Meyer AL, Mohr F-W, Strüber M (2014) Update der kardialen Assistenzsysteme. Z Herz- Thorax- Gefäßchir 28(3):185-195

80. Meyer AL, Malehsa D, Bara C, Budde U, Slaughter MS, Haverich A, Strueber M (2010) Acquired von Willebrand syndrome in patients with an axial flow left ventricular assist device. Circ Heart Fail 3(6):675-681

81. Potapov EV, Stepanenko A, Krabatsch T, Hetzer R (2011) Managing long-term complications of left ventricular assist device therapy. Curr Opin Cardiol 26(3):237-244

82. Teuteberg JJ, Kormos RL, Lockard K, Kunz N, Allen C, Scanlon A, Weaver S, Hall D, Bermudez C, Shullo MA (2013) Management of anticoagulation in patients with mechanical circulatory support using a physician-based versus a pharmacist directed anticoagulation clinic-based strategy. J Heart Lung Transplant 32(4):S294-S295

83. Wever-Pinzon O, Stehlik J, Kfoury AG, Terrovitis JV, Diakos NA, Charitos C, Li DY, Drakos SG (2012) Ventricular assist devices: pharmacological aspects of a mechanical therapy. Pharmacol Ther 134(2):189-199

84. Rossi M, Serraino GF, Jiritano F, Renzulli A (2012) What is the optimal anticoagulation in patients with a left ventricular assist device? Interact Cardiovasc Thorac Surg 15(4):733-740

85. Redfield MM, Chen HH, Borlaug BA, Semigran MJ, Lee KL, Lewis G, LeWinter MM, Rouleau JL, Bull DA, Mann DL, Deswal A, Stevenson LW, Givertz MM, Ofili EO, O'Connor CM, Felker GM, Goldsmith SR, Bart BA, McNulty SE, Ibarra JC, Lin G, Oh JK, Patel MR, Kim RJ, Tracy RP, Velazquez EJ, Anstrom KJ, Hernandez AF, Mascette AM, Braunwald E (2013) Effect of phosphodiesterase-5 inhibition on exercise capacity and clinical status in heart failure with preserved ejection fraction: a randomized clinical trial. JAMA 309(12):1268-1277

86. Simonneau G, Rubin LJ, Galiè N, Barst RJ, Fleming TR, Frost A, Engel P, Kramer MR, Serdarevic-Pehar M, Layton GR, Sitbon O, Badesch DB (2014) Long-term sildenafil added to intravenous epoprostenol in patients with pulmonary arterial hypertension. $\mathbf{J}$ Heart Lung Transplant 33(7):689-697

87. Tedford RJ, Hemnes AR, Russell SD, Wittstein IS, Mahmud M, Zaiman AL, Mathai SC, Thiemann DR, Hassoun PM, Girgis RE, Orens JB, Shah AS, Yuh D, Conte JV, Champion HC (2008) PDE5A inhibitor treatment of persistent pulmonary hypertension after mechanical circulatory support. Circ Heart Fail 1(4):213-219

88. Milano C, Simeone A (2013) Mechanical circulatory support: devices, outcomes and complications. Heart Fail Rev 18(1):35-53
89. Hannan M, Husain S, Mattner F, Danziger-Isakov L, Drew R, Corey GR, Schueler S, Holman W, Lawler L, Gordon S, Mahon NG, Herre J, Gould K, Montoya J, Padera R, Kormos R, Conte J, Mooney M (2011) Working formulation for the standardization of definitions of infections in patients using ventricular assist devices. J Heart Lung Transplant 30(4):375-384

90. Pauwaa S, Bhat G (2011) Ventricular assist device-specific infections. In: Shuhaiber J (Hrsg) Ventricular assist devices. InTechOpen, Rijeka

91. Holman WL, Rayburn BK, McGiffin DC, Foley BA, Benza RL, Bourge RC, Pinderski LJ, Kirklin JK (2003) Infection in ventricular assist devices. Prevention and treatment. Ann Thorac Surg 75(6):S48-S57

92. Sharma V, Deo S, Stulak J, Durham L, Daly R, Park S, Baddour L, Mehra K, Joyce L (2012) Driveline infections in left ventricular assist devices: implications for destination therapy. Ann Thorac Surg 94(5):1381-1386.

93. Nienaber J, Kusne S, Riaz T, Walker R, Baddour L, Wright AJ, Park S, Vikram H, Keating M, Arabia F, Lahr B, Sohail M (2013) Clinical manifestations and management of left ventricular assist device-associated infections. Clin Infect Dis 57(10):1438-1448

94. Ruscher C (2014) Empfehlungen zur Prävention und Kontrolle von Methicillin-resistenten Staphylococcus aureus-Stämmen (MRSA) in medizinischen und pflegerischen Einrichtungen. Bundesgesundheitsbl Gesundheitsforsch Gesundheitsschutz 57(6):695-732

95. Garatti A, Giuseppe B, Russo C, Marco O, Ettore V (2007) Drive-line exit-site infection in a patient with axial-flow pump support: successful management using vacuum-assisted therapy. J Heart Lung Transplant 9(26):956-959

96. World Health Organization (2001) International classification of functioning, disability and health. ICF. WHO, Geneva

97. Knoglinger E (2008) WHO-Klassifikation ICF: Grundlagen und Anwendbarkeit in der kardiologischen Rehabilitation. Herzmedizin 25:36-42

98. Cieza A, Ewert T, Ustün TB, Chatterji S, Kostanjsek N, Stucki G (2004) Development of ICF Core Sets for patients with chronic conditions. J Rehabil Med (44 Suppl):9-11

99. Wildner M, Quittan M, Portenier L, Wilke S, Boldt C, Stucki G, Kostanjsek N, Grill E (2005) ICF Core Set for patients with cardiopulmonary conditions in early post-acute rehabilitation facilities. Disabil Rehabil 27(7-8):397-404

100. Deutsches Institut für Medizinische Dokumentation und Information (DIMDI) (2005)WHO-Kooperationszentrum für das System Internationaler Klassifikationen: Internationale Klassifikation der Funktionsfähigkeit, Behinderung und Gesundheit. http://www.dimdi.de/dynamic/de/klassi/downloadcenter/icf/endfassung/. Zugegriffen: 18. Mai 2015

101. Schüle K, Huber G (2000) Grundlagen der Sporttherapie. Prävention, ambulante und stationäre Rehabilitation, 1. Aufl. Urban \& Fischer, München

102. Maybaum S, Mancini D, Xydas S, Starling RC, Aaronson K, Pagani FD, Miller LW, Margulies K, McRee S, Frazier OH, Torre-Amione G (2007) Cardiac improvement during mechanical circulatory support: a prospective multicenter study of the LVAD Working Group. Circulation 115(19):2497-2505

103. Rogers J, Aaronson K, Boyle A, Russell S, Milano C, Pagani F, Edwards B, Park S, John R, Conte J, Farrar D, Slaughter M (2010) Continuous flow left ventricular assist device improves functional capacity and quality of life of advanced heart failure patients. J Am Coll Cardiol 55(17):1826-1834

104. Jung MH, Gustafsson F (2015) Exercise in heart failure patients supported with a left ventricular assist device. J Heart Lung Transplant 34(4):489-496

105. Humphrey R (1997) Exercise physiology in patients with left ventricular assist devices. J Cardpulm Rehabil 17(2):73-75 
106. Piña I (2010) Cardiac rehabilitation in heart failure: a brief review and recommendations. Curr Cardiol Rep 12(3):223-229

107. Wells C (2013) Physical therapist management of patients with ventricular assist devices: key considerations for the acute care physical therapist. Phys Ther 93(2):266-278

108. Hayes K, Leet A, Bradley S, Holland A (2012) Effects of exercise training on exercise capacity and quality of life in patients with a left ventricular assist device: a preliminary randomized controlled trial. J Heart Lung Transplant 31(7):729-734

109. Karapolat H, Engin C, Eroglu M, Yagdi T, Zoghi M, Nalbantgil S, Durmaz B, Kirazli Y, Ozbaran M (2013) Efficacy of the cardiac rehabilitation program in patients with end-stage heart failure, heart transplant patients, and left ventricular assist device recipients. Transplant Proc 45(9):3381-3385

110. Kohli H, Canada J, Arena R, Tang D, Peberdy M, Harton S, Flattery M, Doolin K, Katlaps G, Hess M, Kasirajan V, Shah K (2011) Exercise blood pressure response during assisted circulatory support: comparison of the total artifical heart with a left ventricular assist device during rehabilitation. J Heart Lung Transplant 30(11):1207-1213

111. Laoutaris I, Dritsas A, Adamopoulos S, Manginas A, Gouziouta A, Kallistratos M, Koulopoulou M, Voudris V, Cokkinos D, Sfirakis P (2011) Benefits of physical training on exercise capacity, inspiratory muscle function, and quality of life in patients with ventricular assist devices long-term postimplantation. Eur $\mathrm{J}$ Cardiovasc Prev Rehabil 18(1):33-40

112. Marko C, Danzinger G, Käferbäck M, Lackner T, Müller R, Zimpfer D, Schima H, Moscato F (2014) Safety and efficacy of cardiac rehabilitation for patients with continuous flow left ventricular assist devices. Eur J Prev Cardiol 22(11):1378-1384

113. Nissinoff J, Tian F, Therattil M, Salvarrey R, Lee S (2011) Acute inpatient rehabilitation after left ventricular assist device implantation for congestive heart failure. PM R 3(6):586-589

114. Kugler C, Malehsa D, Schrader E, Tegtbur U, Guetzlaff E, Haverich A, Strueber M (2012) A multi-modal intervention in management of left ventricular assist device outpatients: dietary counselling, controlled exercise and psychosocial support. Eur J Cardiothorac Surg 42(6): 1026-1032

115.Alsara O, Perez-Terzic C, Squires RW, Dandamudi S, Miranda WR, Park SJ, Thomas RJ (2014) Is exercise training safe and beneficial in patients receiving left ventricular assist device therapy? J Cardiopulm Rehabil Prev 34(4):233-240

116. Compostella L, Russo N, Setzu T, Bottio T, Compostella C, Tarzia V, Livi U, Gerosa G, Iliceto S, Bellotto F (2015) A practical review for cardiac rehabilitation professionals of continuous-flow left ventricle assist devices: historical and current perspectives. J Cardiopulm Rehabil Prev 35(5):301-311

117. Workowski A (2015) Kardiologische Rehabilitation von Patienten mit einem Herzunterstützungssystem. Dissertation, Deutsche Sporthochschule Köln

118. Workowski A, Bartsch P, Stuhr H, Willemsen D, BjarnasonWehrens B (2014) Resistance training in patients with an intracorporal ventricular assist device (VAD) of the third generation during phase II cardiac rehabilitation (CR). Eur J Prev Cardiol 21:6

119. Piepoli M, Conraads V, Corrà U, Dickstein K, Francis D, Jaarsma T, McMurray J, Pieske B, Piotrowicz E, Schmid J, Anker S, Solal A, Filippatos G, Hoes A, Gielen S, Giannuzzi P, Ponikowski P (2011) Exercise training in heart failure: from theory to practice. A consensus document of the Heart Failure Association and the European Association for Cardiovascular Prevention and Rehabilitation. Europ J Heart Fail 13(4):347-357
120. Tagermann M, Russel N (2009) Standard of care: physical therapy management of the patient with a ventricular assist device. http://www.brighamandwomens.org/Patients_Visitors/pcs/ rehabilitationservices/Physical $\% 20$ Therapy $\% 20$ Standards $\% 20$ of $\% 20$ Care $\% 20$ and $\% 20$ Protocols/Cardiac\%20-\%20VAD $\% 20$ SOC\%20.pdf. Zugegriffen: 13. Mai 2015

121. Roefe D, Schulz U (2014) „Ventricular-assist-device“Koordination. Z Herz- Thorax- Gefäßchir 28(5):327-331

122. Gemeinsamer Bundesausschuss (2011) Richtlinie des Gemeinsamen Bundesausschusses über die Verordnung von Heilmitteln in der vertragsärztlichen Versorgung: HeilmittelRichtlinie. www.g-ba.de/downloads/62-492-532/HeilMRL 2011-05-19 bf.pdf. Zugegriffen: 5. März 2015

123. Granger CV, Hamilton BB, Linacre JM, Heinemann AW, Wright BD (1993) Performance profiles of the functional independence measure. Am J Phys Med Rehabil 72(2):84-89

124. Internationale Vereinigung für Assessment in der Rehabilitation (IVAR) (Hrsg) FIM - Funktionale Selbständigkeitsmessung. Manual. 1. Version. Straubing 1997

125. Shepherd J, Wilding C (2006) Occupational therapy for people with ventricular assist devices. Aust Occup Therap J 53(1):47-49

126. Albert W, Bittner A, Kiekbusch S, Buschtöns C, Gehringer A, Kinzel S, Hetzer R (2002) Das Kunstherz - eine extreme psychische Belastungssituation? Z Herz Thorax Gefasschir 16(1): $\mathrm{I} / 110-\mathrm{I} / 119$

127. Bunzel B, Roethy W, Znoj H, Laederach-Hofmann K (2008) Psychological consequences of life-saving cardiac surgery in patients and partners: measurement of emotional stress by the Impact of Event Scale. Stress Health 24(5):351-363

128. Mapelli, D.; Cavazzana, A.; Cavalli, C.; Bottio, T.; Tarzia, V.; Gerosa, G.; Volpe, B. R.: Clinical psychological and neuropsychological issues with left ventricular assist devices (LVADs). In: Annals of cardiothoracic surgery 3 (2014) 5, S. 480-89.

129. Maciver J, Ross H (2012) Quality of life and left ventricular assist device support. Circulation 126(7):866-874

130. Rose EA, Gelijns AC, Moskowitz AJ, Heitjan DF, Stevenson LW, Dembitsky W, Long JW, Ascheim DD, Tierney AR, Levitan RG, Watson JT, Meier P, Ronan NS, Shapiro PA, Lazar RM, Miller LW, Gupta L, Frazier OH, Desvigne-Nickens P, Oz MC, Poirier VL (2001) Long-term use of a left ventricular assist device for end-stage heart failure. N Engl J Med 345(20):1435-1443

131. Brouwers C, Denollet J, De Jonge N, Caliskan K, Kealy J, Pedersen S (2011) Patient-reported outcomes in left ventricular assist device therapy: a systematic review and recommendations for clinical research and practice. Circ Heart Fail 4(6):714-723

132. Kugler C, Malehsa D, Tegtbur U, Guetzlaff E, Meyer A, Bara C, Haverich A, Strueber M (2011) Health-related quality of life and exercise tolerance in recipients of heart transplants and left ventricular assist devices: a prospective, comparative study. J Heart Lung Transplant 30(2):204-210

133. Ladwig K, Lederbogen F, Albus C, Angermann C, Borggrefe M, Fischer D, Fritzsche K, Haass M, Jordan J, Jünger J, Kindermann I, Köllner V, Kuhn B, Scherer M, Seyfarth M, Völler H, Waller C, Herrmann-Lingen C (2013) Positionspapier zur Bedeutung psychosozialer Faktoren in der Kardiologie. Kardiologe 7(1):7-27

134. Petrucci R, Rogers J, Blue L, Gallagher C, Russell S, Dordunoo D, Jaski B, Chillcott S, Sun B, Yanssens T, Tatooles A, Koundakjian L, Farrar D, Slaughter M (2012) Neurocognitive function in destination therapy patients receiving continuousflow vs pulsatile-flow left ventricular assist device support. J Heart Lung Transplant 31(1):27-36 
135. Kirklin J, Naftel D, Kormos R, Stevenson L, Pagani F, Miller M, Timothy Baldwin J, Young J (2013) Fifth INTERMACS annual report: risk factor analysis from more than 6,000 mechanical circulatory support patients. J Heart Lung Transplant 32(2):141-156

136. Jorde UP, Kushwaha SS, Tatooles AJ, Naka Y, Bhat G, Long JW, Horstmanshof DA, Kormos RL, Teuteberg JJ, Slaughter MS, Birks EJ, Farrar DJ, Park SJ (2014) Results of the destination therapy post-food and drug administration approval study with a continuous flow left ventricular assist device: a prospective study using the INTERMACS registry (Interagency Registry for Mechanically Assisted Circulatory Support). J Am Coll Cardiol 63(17):1751-1757

137. Herrmann-Lingen C, Buss U, Snaith R (2011) HADS-D Hopital Anxiety and Depression Scale - Deutsche Version. 3. aktualisierte und neu normierte Aufl. Hogrefe, Bern

138. Bullinger M, Kirchberger I (1998) Medical outcomes study Short-Form General Health Survey SF-36 manual (German version). Göttingen

139. Graf J-M, Claes C, Greiner W, Uber A (1998) Die deutsche Version des EuroQol-Fragebogens. J Public Health 6(1):3-20

140. Rabin R, Charro F de (2001) EQ-5D: a measure of health status from the EuroQol Group. Ann Med 33(5):337-343

141. Faller H, Steinbüchel T, Schowalter M, Spertus JA, Störk S, Angermann CE (2005) Der Kansas City Cardiomyopathy Questionnaire (KCCQ) - ein neues krankheitsspezifisches Messinstrument zur Erfassung der Lebensqualität bei chronischer Herzinsuffizienz - Psychometrische Prüfung der deutschen Version. Psychother Psychosom Med Psychol 55(3-4):200-208

142. Green C, Porter CB, Bresnahan DR, Spertus JA (2000) Development and evaluation of the Kansas City Cardiomyopathy Questionnaire. A new health status measure for heart failure. J Am Coll Cardiol 35(5):1245-1255

143. Middel B, Bouma J, Jongste M de, van Sonderen E, Niemeijer MG, Crijns H, van den Heuvel W (2001) Psychometric properties of the Minnesota Living with Heart Failure Questionnaire (MLHF-Q). Clin Rehabil 15(5):489-500

144. Quittan M, Wiesinger GF, Crevenna R, Nuhr MJ, Posch M, Hülsmann M, Müller D, Pacher R, Fialka-Moser V (2001) Crosscultural adaptation of the Minnesota Living with Heart Failure Questionnaire for German-speaking patients. J Rehabil Med 33(4):182-186

145. Lietz K, Long JW, Kfoury AG, Slaughter MS, Silver MA, Milano CA, Rogers JG, Naka Y, Mancini D, Miller LW (2007) Outcomes of left ventricular assist device implantation as destination therapy in the post-REMATCH era: implications for patient selection. Circulation 116(5):497-505

146. Dang N, Topkara V, Kim B, Lee B, Remoli R, Naka Y (2005) Nutritional status in patients on left ventricular assist device support. J Thorac Cardiovasc Surg 130(5):e3-e4

147. John R, Lietz K, Schuster M, Naka Y, Rao V, Mancini DM, Rose EA, Smith CR, Oz MC, Edwards NM, Itescu S (2003) Immunologic sensitization in recipients of left ventricular assist devices. J Thorac Cardiovasc Surg 125(3):578-591

148. Mano A, Fujita K, Uenomachi K, Kazama K, Katabuchi M, Wada K, Terakawa N, Arai K, Hori Y, Hashimoto S, Nakatani T, Kitamura S (2009) Body mass index is a useful predictor of prognosis after left ventricular assist system implantation. J Heart Lung Transplant 28(5):428-433

149. Evans W, Morley J, Argilés J, Bales C, Baracos V, Guttridge D, Jatoi A, Kalantar-Zadeh K, Lochs H, Mantovani G, Marks D, Mitch W, Muscaritoli M, Najand A, Ponikowski P, Rossi Fanelli F, Schambelan M, Schols A, Schuster M, Thomas D, Wolfe R, Anker S (2008) Cachexia: a new definition. Clin Nutr 27(6):793-799
150. Ng MFY (2010) Cachexia - an intrinsic factor in wound healing. Int Wound J 7(2):107-113

151. Anker SD, Negassa A, Coats AJS, Afzal R, Poole-Wilson PA, Cohn JN, Yusuf S (2003) Prognostic importance of weight loss in chronic heart failure and the effect of treatment with angiotensinconverting-enzyme inhibitors. An observational study. Lancet 361(9363):1077-1083

152. Lietz K, John R, Burke EA, Ankersmit JH, McCue JD, Naka Y, Oz MC, Mancini DM, Edwards NM (2001) Pretransplant cachexia and morbid obesity are predictors of increased mortality after heart transplantation1. Transplantation 72(2):277-283

153. McMurray J, Petrie M, Swedberg K, Komajda M, Anker S, Gardner R (2009) Heart failure. In: Camm A, Lüscher T, Serruys P (Hrsg) The ESC textbook of cardiovascular medicine. Oxford University Press, Rotterdam

154. Sandek A, Doehner W, Anker SD, Haehling S von (2009) Nutrition in heart failure: an update. Curr Opin Clin Nutr Metab Care 12(4):384-391

155. Montgomery T, Cohen A, Garnick J, Spadafore J, Boldea E (2012) Nutrition assessment, care, and considerations of ventricular assist device patients. Nutr Clin Pract 27(3):352-362

156. Akashi YJ, Springer J, Anker SD (2005) Cachexia in chronic heart failure. Prognostic implications and novel therapeutic approaches. Curr Heart Fail Rep 2(4):198-203

157. Pasini E, Opasich C, Pastoris O, Aquilani R (2004) Inadequate nutritional intake for daily life activity of clinically stable patients with chronic heart failure. Am J Cardiol 93(8A):41A-43A

158. Nestlé Nutrition Institute: Mini Nutritional Assessment. MNA ${ }^{\circledR}$. http://www.mnaelderly.com/forms/mini/mna_mini_german.pdf. Zugegriffen: 26. Juni 2015

159. Deutsche Adipositas-Gesellschaft(DAG)e. V., Deutsche Diabetes Gesellschaft (DDG), Deutsche Gesellschaft für Ernährung (DGE) e. V., Deutsche Gesellschaft für Ernährungsmedizin (DGEM) e. V. (2014): Interdisziplinäre Leitlinie der Qualität S3 zur „Prävention und Therapie der Adipositas“, Version 2.0 (April 2014). http://www.awmf.org/uploads/tx_szleitlinien/050-0011_ S3_Adipositas_Prävention_Therapie_2014-11.pdf. Zugegriffen: 26. Juni 2015

160. Creutzberg EC, Wouters EFM, Mostert R, Pluymers RJ, Schols AMWJ (2003) A role for anabolic steroids in the rehabilitation of patients with COPD? A double-blind, placebo-controlled, randomized trial. Chest 124(5):1733-1742

161. Slaughter MS, Pappas P, Tatooles A (2003) Percutaneous endoscopic gastrostomy tube in a patient with a left ventricular assist device. ASAIO J 49(5):611-612

162. Kenchaiah S, Evans JC, Levy D, Wilson PWF, Benjamin EJ, Larson MG, Kannel WB, Vasan RS (2002) Obesity and the risk of heart failure. N Engl J Med 347(5):305-313

163. Yancy C, Jessup M, Bozkurt B, Butler J, Casey D, Drazner M, Fonarow G, Geraci S, Horwich T, Januzzi J, Johnson M, Kasper E, Levy W, Masoudi F, McBride P, McMurray J, Mitchell J, Peterson P, Riegel B, Sam F, Stevenson L, Tang W, Tsai E, Wilkoff B (2013) 2013 AHA guideline for the management of heart failure. J Am Coll Cardiol 62(16):e147-e239

164. Habbu A, Lakkis NM, Dokainish H (2006) The obesity paradox: fact or fiction? Am J Cardiol 98(7):944-948

165. Musci M, Loforte A, Potapov EV, Krabatsch T, Weng Y, Pasic M, Hetzer R (2008) Body mass index and outcome after ventricular assist device placement. Ann Thorac Surg 86(4):1236-1242

166. Klodell CT, Staples ED, Aranda JM, Schofield RS, Hill JA, Pauly DF, Beaver TM (2006) Managing the post-left ventricular assist device patient. Congest Heart Fail 12(1):41-45

167. MTBASA - Medizinisch-Technische Beratungsstelle der Arbeitsgemeinschaft Selbstkontrolle der Antikoagulation. http://www. mtbasa.de. Zugegriffen: 15. Jan 2016 
168. Hiestand B (2009) Circulatory assist devices in heart failure patients. Heart Fail Clin 5(1):55-62

169. Bundesärztekammer (BÄK); Arbeitsgemeinschaft der Deutschen Ärztekammern; Kassenärztliche Bundesvereinigung (KBV); Arbeitsgemeinschaft der Wissenschaftlichen Medizinischen Fachgesellschaften (AWMF) (2009): Nationale Versorgungsleitlinie „Chronische Herzinsuffizienz“ - Langfassung, 1. Aufl. Version 7. 2009, zuletzt geändert: August 2013. http://www.leitlinien.de/mdb/downloads/nvl/herzinsuffizienz/herzinsuffizienz1aufl-vers7-lang.pdf. Zugegriffen: 21. Mai 2015

170. Universität Leipzig - Herzzentrum Kunstherz-Patientenführerschein

171. Corra U, Pistono M, Piepoli MF, Giannuzzi P (2012) Ventricular assist device patients on the horizon of cardiovascular prevention and rehabilitation. Can we convert challenges into opportunities? Eur J Prev Cardiol 19(3):490-493

172. Egerod I, Overgaard D (2012) Taking a back seat: support and self-preservation in close relatives of patients with left ventricular assist device. Eur J Cardiovasc Nurs 11(4):380-387

173. Nguyen E, Stein J (2013) Functional outcomes of adults with left ventricular assist devices receiving inpatient rehabilitation. PM R 5(2):99-103

174. Ambardekar A, Cannon A, Cleveland J, Brieke A, Lindenfeld J (2011) Driving with a driveline: a survey of current practice patterns for allowing a patient supported with a left ventricular assist device to drive. J Heart Lung Transplant 30(10):1204-1205

175. Thoratec Corporation (2011) Heart Mate II community living manual: your guide to successful LVAS patient discharges. Thoracec Corp, Pleasanton

176. Emani S, O'Keefe C, Wissman S, MacBrair K, White L, Hasan A, Sai-Sudhakar C, Sun B (2011) 190 Driver's education: a single center experience on the incidence and safety of driving with LVADS. J Heart Lung Transplant 30(4):S70

177. Emani S, Pagani F, Eckman P, Gordon R, Brisco M, Klodell C, Mandras S, Hasan A, Sai-Sudhakar C, Sun B (2013) Hit the road: a multicenter evaluation of driving with LVADs. J Heart Lung Transplant 32(4):S98

178. Klein H, Krämer A, Pieske B, Trappe H, De Vries H (2010) Fahreignung bei kardiovaskulären Erkrankungen. Kardiologe 4(6):441-473

179. Golka K, Hengstler JG (Hrsg) (2011) Verkehrsmedizin - arbeitsmedizinische Aspekte. Orientierungshilfe für Praxis, Klinik und Betrieb; Schwerpunktthema Jahrestagung DGAUM 2010. ecomed, Heidelberg

180. Madea B, Abel R (Hrsg) (2012) Verkehrsmedizin. Fahreignung, Fahrsicherheit, Unfallrekonstruktion; mit 128 Tabellen. Deutscher Ärzte-Verlag, Köln

181. Baskett R, Crowell R, Freed D, Giannetti N, Simpson C (2012) Canadian Cardiovascular Society focused position statement update on assessment of the cardiac patient for fitness to drive: fitness following left ventricular assist device implantation. Can J Cardiol 28(2):137-140

182. Gräcmann N, Albrecht M Begutachtungsleitlinien zur Kraftfahreignung. Berichte der Bundesanstalt für Straßenwesen, Mensch und Sicherheit Heft M 115, gültig ab 1. Mai 2014. Bergisch Gladbach 2014

183. Coyle L, Martin M, Kurien S, Graham J, Gallagher C, Silver M, Slaughter M (2008) Destination therapy: safety and feasibility of national and international travel. ASAIO J 54(2):172-176

184. Lufthansa Operation Center, m. 0.-5., mündliche Information

185. MedStar Heart Institute Air Travel Letter Template. http://www. mylvad.com/content/lvad-travel-tips. Zugegriffen: 21. Mai 2015

186. Park S, Milano C, Tatooles A, Rogers J, Adamson R, Steidley D, Ewald G, Sundareswaran K, Farrar D, Slaughter M (2012) Outcomes in advanced heart failure patients with left ventricular assist devices for destination therapy. Circ Heart Fail 5(2):241-248
187. Bunzel B, Laederach-Hofmann K, Wieselthaler G, Roethy W, Wolner E (2007) Mechanical circulatory support as a bridge to heart transplantation: what remains? Long-term emotional sequelae in patients and spouses. J Heart Lung Transplant 26(4):384-389

188. Dev S, Abernethy A, Rogers J, O’Connor C (2012) Preferences of people with advanced heart failure - a structured narrative literature review to inform decision making in the palliative care setting. Am Heart J 164(3):313-319.e5

189. Bruce C, Smith M, McCullough L (2013) Clarification of the intent of ventricular assist devices before patient consent. J Thorac Cardiovasc Surg 145(6):1423-1425

190. Bramstedt K, Chalfant A, Wright C (2007) Emergency consults in the setting of transplant medicine. Dilemmas for social workers and bioethicists. Prog Transplant 17(1):36-39

191. Bruce C, Bhimaraj A, Smith M (2014) Revisiting surrogate consent for ventricular assist device placement. Ann Thorac Surg 97(3):747-749

192. Brush S, Budge D, Alharethi R, McCormick A, MacPherson J, Reid B, Ledford I, Smith H, Stoker S, Clayson S, Doty J, Caine W, Drakos S, Kfoury A (2010) End-of-life decision making and implementation in recipients of a destination left ventricular assist device. J Heart Lung Transplant 29(12):1337-1341

193. Tanner C, Fromme E, Goodlin S (2011) Ethics in the treatment of advanced heart failure: palliative care and end-of-life issues. Congest Heart Fail 17(5):235-240

194. Adler E, Goldfinger J, Kalman J, Park M, Meier D (2009) Palliative care in the treatment of advanced heart failure. Circulation 120(25):2597-2606

195. Kini V, Kirkpatrick J (2013) Ethical challenges in advanced heart failure. Curr Opin Support Palliat Care 7(1):21-28

196. Swetz K, Freeman M, AbouEzzeddine O, Carter K, Boilson B, Ottenberg A, Park S, Mueller P (2011) Palliative medicine consultation for preparedness planning in patients receiving left ventricular assist devices as destination therapy. Mayo Clin Proc 86(6):493-500

197. Gerhard C et al (2012) Arbeitspapier der AG Nicht-Tumorpatienten - Palliativversorgung bei Herzinsuffizienz. Z Palliativmed 13(02):61-63

198. Jaarsma T, Beattie J, Ryder M, Rutten F, McDonagh T, Mohacsi P, Murray S, Grodzicki T, Bergh I, Metra M, Ekman I, Angermann C, Leventhal M, Pitsis A, Anker S, Gavazzi A, Ponikowski P, Dickstein K, Delacretaz E, Blue L, Strasser F, McMurray J (2009) Palliative care in heart failure: a position statement from the palliative care workshop of the Heart Failure Association of the European Society of Cardiology. Eur J Heart Fail 11(5):433-443

199. Bruce CR, Brody B, Majumder MA (2013) Ethical dilemm as surrounding the use of ventricular assist devices in supporting patients with end-stage organ dysfunction. Methodist Debakey Cardiovase J 9(1):11-14

200. Rizzieri A, Verheijde J, Rady M, McGregor J (2008) Ethical challenges with the left ventricular assist device as a destination therapy. Philos Ethics Humanit Med 3(1):20

201. Bundesärztekammer (2011) Grundsätze der Bundesärztekammer zur ärztlichen Sterbebegleitung. Dtsch Arztebl 108(7):A346-A348

202. Bramstedt K (2008) Destination nowhere: a potential dilemma with ventricular assist devices. ASAIO J 54(1):1-2

203. Mueller P, Swetz K, Freeman M, Carter K, Crowley M, Severson C, Park S, Sulmasy D (2010) Ethical analysis of withdrawing ventricular assist device support. Mayo Clin Proc 85(9):791-797

204. Gafford E, Luckhardt A, Swetz K (2013) Deactivation of a left ventricular assist device at the end of life \#269. J Palliat Med 16(8):980-982 